\title{
Quaternary glaciation and the Great American Biotic Interchange
}

\section{Bacon et al.}

Appendix 1. Abundance of mammal fossil occurrences found that migrated across the Isthmus of Panama through time.

\begin{tabular}{|c|c|c|c|c|c|c|c|c|c|}
\hline PBDB \# / publication & Collection authorizer & Order & Family & Occurrence - Genus & Occurrence - Species & Country & Max age & Min age & Direction \\
\hline 63340 & J. Alroy & A Artiodactlya & Bovidae & Bos & taurus & Argentina & "0,01 & 0,00 & NA-SA \\
\hline 63343 & D. Croft & Artiodactlya & Bovidae & Bos & taurus & Argentina & 0,01 & 0,00 & NA-SA \\
\hline 63344 & D. Croft & Artiodactlya & Bovidae & Bos & taurus & Argentina & 0,01 & 0,00 & NA-SA \\
\hline 71329 & J. Alroy & Artiodactlya & Bovidae & Bos & taurus & Peru & 0,01 & 0,01 & NA-SA \\
\hline 71329 & J. Alroy & Artiodactlya & Bovidae & Ovis & sp. & Peru & 0,01 & 0,01 & NA-SA \\
\hline 71303 & J. Alroy & Artiodactlya & Camelidae & Eulamaops & paralellus & Argentina & 0,78 & 0,01 & NA-SA \\
\hline 71303 & J. Alroy & Artiodactlya & Camelidae & Hemiauchenia & paradoxa & Argentina & 0,78 & 0,01 & NA-SA \\
\hline 114698 & M. Uhen & Artiodactlya & Camelidae & Hemiauchenia & paradoxa & Argentina & 0,13 & 0,01 & NA-SA \\
\hline 123831 & M. Uhen & Artiodactlya & Camelidae & Hemiauchenia & paradoxa & Argentina & 2,59 & 0,01 & NA-SA \\
\hline 142016 & C. Jaramillo & Artiodactlya & Camelidae & Hemiauchenia & paradoxa & Bolivia & 2,59 & 0,78 & NA-SA \\
\hline 148419 & C. Jaramillo & Artiodactlya & Camelidae & Hemiauchenia & paradoxa & Brazil & 0,13 & 0,01 & NA-SA \\
\hline 149093 & C. Jaramillo & Artiodactlya & Camelidae & Hemiauchenia & paradoxa & Brazil & 0,13 & 0,01 & NA-SA \\
\hline 149143 & C. Jaramillo & Artiodactlya & Camelidae & Hemiauchenia & paradoxa & Brazil & 0,13 & 0,01 & NA-SA \\
\hline Kerber et al. 2014 & M. Vallejo & Artiodactlya & Camelidae & Hemiauchenia & paradoxa & Brazil & 0,04 & 0,01 & NA-SA \\
\hline Pino et al. 2013 & M. Vallejo & Artiodactlya & Camelidae & Hemiauchenia & paradoxa & Chile & 0,01 & 0,01 & NA-SA \\
\hline 71298 & J. Alroy & Artiodactlya & Camelidae & Hemiauchenia & sp. & Brazil & 2,59 & 0,01 & NA-SA \\
\hline 71301 & J. Alroy & Artiodactlya & Camelidae & Hemiauchenia & sp. & Brazil & 2,59 & 0,01 & NA-SA \\
\hline 71302 & J. Alroy & Artiodactlya & Camelidae & Hemiauchenia & sp. & Brazil & 2,59 & 0,01 & NA-SA \\
\hline 71316 & J. Alroy & Artiodactlya & Camelidae & Hemiauchenia & sp. & Argentina & 0,13 & 0,01 & NA-SA \\
\hline 120884 & M. Uhen & Artiodactlya & Camelidae & Hemiauchenia & sp. & Uruguay & 0,78 & 0,01 & NA-SA \\
\hline 142016 & C. Jaramillo & Artiodactlya & Camelidae & Lama & castelnaudi & Bolivia & 2,59 & 0,78 & NA-SA \\
\hline 70673 & D. Croft & Artiodactlya & Camelidae & Lama & glama & Bolivia & 2,59 & 0,78 & NA-SA \\
\hline 140713 & C. Jaramillo & Artiodactlya & Camelidae & Lama & glama & Argentina & 0,78 & 0,01 & NA-SA \\
\hline Kerber et al. 2014 & M. Vallejo & Artiodactlya & Camelidae & Lama & gracilis & Brazil & 0,04 & 0,01 & NA-SA \\
\hline Kerber et al. 2014 & M. Vallejo & Artiodactlya & Camelidae & Lama & guanicoe & Brazil & 0,04 & 0,01 & NA-SA \\
\hline 63337 & J. Alroy & Artiodactlya & Camelidae & Lama & guanicoe & Argentina & 0,78 & 0,01 & NA-SA \\
\hline 63338 & J. Alroy & Artiodactlya & Camelidae & Lama & guanicoe & Argentina & 0,01 & 0,00 & NA-SA \\
\hline 63339 & J. Alroy & Artiodactlya & Camelidae & Lama & guanicoe & Argentina & 0,01 & 0,00 & NA-SA \\
\hline 63340 & J. Alroy & Artiodactlya & Camelidae & Lama & guanicoe & Argentina & 0,01 & 0,00 & NA-SA \\
\hline 63341 & D. Croft & Artiodactlya & Camelidae & Lama & guanicoe & Argentina & 0,13 & 0,00 & NA-SA \\
\hline 63345 & D. Croft & Artiodactlya & Camelidae & Lama & guanicoe & Argentina & 0,13 & 0,00 & NA-SA \\
\hline 73843 & J. Alroy & Artiodactlya & Camelidae & Lama & guanicoe & Argentina & 0,01 & 0,00 & NA-SA \\
\hline 73844 & J. Alroy & Artiodactlya & Camelidae & Lama & guanicoe & Argentina & 0,13 & 0,01 & NA-SA \\
\hline 73845 & J. Alroy & Artiodactlya & Camelidae & Lama & guanicoe & Argentina & 0,13 & 0,01 & NA-SA \\
\hline 63346 & D. Croft & Artiodactlya & Camelidae & Lama & guanicoe & Argentina & 0,01 & 0,00 & NA-SA \\
\hline 71286 & J. Alroy & Artiodactlya & Camelidae & Lama & guanicoe & Argentina & 0,78 & 0,01 & NA-SA \\
\hline 71287 & J. Alroy & Artiodactlya & Camelidae & Lama & guanicoe & Argentina & 0,78 & 0,01 & NA-SA \\
\hline 71303 & J. Alroy & Artiodactlya & Camelidae & Lama & guanicoe & Argentina & 0,78 & 0,01 & NA-SA \\
\hline 63347 & D. Croft & Artiodactlya & Camelidae & Lama & guanicoe & Argentina & 0,01 & 0,00 & NA-SA \\
\hline 71313 & J. Alroy & Artiodactlya & Camelidae & Lama & guanicoe & Brazil & 0,02 & 0,02 & NA-SA \\
\hline 71314 & J. Alroy & Artiodactlya & Camelidae & Lama & guanicoe & Argentina & 0,78 & 0,01 & NA-SA \\
\hline 71336 & J. Alroy & Artiodactlya & Camelidae & Lama & guanicoe & Argentina & 0,13 & 0,01 & NA-SA \\
\hline 71337 & J. Alroy & Artiodactlya & Camelidae & Lama & guanicoe & Argentina & 0,01 & 0,01 & NA-SA \\
\hline 71338 & J. Alroy & Artiodactlya & Camelidae & Lama & guanicoe & Argentina & 0,13 & 0,01 & NA-SA \\
\hline 71339 & J. Alroy & Artiodactlya & Camelidae & Lama & guanicoe & Argentina & 0,13 & 0,01 & NA-SA \\
\hline 71341 & J. Alroy & Artiodactlya & Camelidae & Lama & guanicoe & Chile & 0,01 & 0,01 & NA-SA \\
\hline 71342 & J. Alroy & Artiodactlya & Camelidae & Lama & guanicoe & Chile & 0,13 & 0,01 & NA-SA \\
\hline 71343 & J. Alroy & Artiodactlya & Camelidae & Lama & guanicoe & Argentina & 0,01 & 0,01 & NA-SA \\
\hline 71344 & J. Alroy & Artiodactlya & Camelidae & Lama & guanicoe & Chile & 0,13 & 0,01 & NA-SA \\
\hline 71347 & J. Alroy & Artiodactlya & Camelidae & Lama & guanicoe & Argentina & 0,78 & 0,01 & NA-SA \\
\hline 140934 & C. Jaramillo & Artiodactlya & Camelidae & Lama & guanicoe & Argentina & 0,78 & 0,01 & NA-SA \\
\hline 141367 & C. Jaramillo & Artiodactlya & Camelidae & Lama & guanicoe & Chile & 0,13 & 0,01 & NA-SA \\
\hline 142016 & C. Jaramillo & Artiodactlya & Camelidae & Lama & intermedia & Bolivia & 2,59 & 0,78 & NA-SA \\
\hline 142016 & C. Jaramillo & Artiodactlya & Camelidae & Lama & mesolithica & Bolivia & 2,59 & 0,78 & NA-SA \\
\hline 70673 & D. Croft & Artiodactlya & Camelidae & Lama & oweni & Bolivia & 2,59 & 0,78 & NA-SA \\
\hline 70673 & D. Croft & Artiodactlya & Camelidae & Lama & provicugna & Bolivia & 2,59 & 0,78 & NA-SA \\
\hline 71265 & J. Alroy & Artiodactlya & Camelidae & Lama & sp. & Chile & 0,13 & 0,01 & NA-SA \\
\hline 61305 & D. Croft & Artiodactlya & Camelidae & Lama & sp. & Argentina & 2,59 & 0,01 & NA-SA \\
\hline 63335 & D. Croft & Artiodactlya & Camelidae & Lama & sp. & Argentina & 0,78 & 0,13 & NA-SA \\
\hline 71266 & J. Alroy & Artiodactlya & Camelidae & Lama & sp. & Chile & 0,01 & 0,01 & NA-SA \\
\hline 71272 & J. Alroy & Artiodactlya & Camelidae & Lama & sp. & Argentina & 0,01 & 0,01 & NA-SA \\
\hline 71273 & J. Alroy & Artiodactlya & Camelidae & Lama & sp. & Argentina & 0,00 & 0,00 & NA-SA \\
\hline 71279 & J. Alroy & Artiodactlya & Camelidae & Lama & sp. & Argentina & 0,78 & 0,01 & NA-SA \\
\hline 71286 & J. Alroy & Artiodactlya & Camelidae & Lama & sp. & Argentina & 0,78 & 0,01 & NA-SA \\
\hline 71287 & J. Alroy & Artiodactlya & Camelidae & Lama & sp. & Argentina & 0,78 & 0,01 & NA-SA \\
\hline 71298 & J. Alroy & Artiodactlya & Camelidae & Lama & sp. & Brazil & 2,59 & 0,01 & NA-SA \\
\hline 71301 & J. Alroy & Artiodactlya & Camelidae & Lama & sp. & Brazil & 2,59 & 0,01 & NA-SA \\
\hline 71302 & J. Alroy & Artiodactlya & Camelidae & Lama & sp. & Brazil & 2,59 & 0,01 & NA-SA \\
\hline 71304 & J. Alroy & Artiodactlya & Camelidae & Lama & sp. & Argentina & 2,59 & 0,01 & NA-SA \\
\hline 71590 & D. Croft & Artiodactlya & Camelidae & Lama & sp. & Bolivia & 2,59 & 0,01 & NA-SA \\
\hline 142158 & C. Jaramillo & Artiodactlya & Camelidae & Lama & sp. & Bolivia & 0,13 & 0,01 & NA-SA \\
\hline 142160 & C. Jaramillo & Artiodactlya & Camelidae & Lama & sp. & Bolivia & 0,13 & 0,01 & NA-SA \\
\hline 145576 & C. Jaramillo & Artiodactlya & Camelidae & Lama & sp. & Peru & 2,59 & 0,01 & NA-SA \\
\hline 146602 & C. Jaramillo & Artiodactlya & Camelidae & Lama & sp. & Peru & 0,02 & 0,01 & NA-SA \\
\hline 133630 & C. Jaramillo & Artiodactlya & Camelidae & Palaeolama & aequatorialis & Ecuador & 0,78 & 0,01 & NA-SA \\
\hline 133659 & C. Jaramillo & Artiodactlya & Camelidae & Palaeolama & crassa & Ecuador & 2,59 & 0,01 & NA-SA \\
\hline
\end{tabular}




\begin{tabular}{|c|c|c|c|c|c|c|c|c|c|}
\hline 142016 & C. Jaramillo & Artiodactlya & Camelidae & Palaeolama & crequii & Bolivia & 2,59 & 0,78 & NA-SA \\
\hline 92814 & J. Alroy & Artiodactlya & Camelidae & Palaeolama & major & Brazil & 2,59 & 0,00 & NA-SA \\
\hline 71332 & J. Alroy & Artiodactlya & Camelidae & Palaeolama & major & Brazil & 0,13 & 0,01 & NA-SA \\
\hline 148309 & C. Jaramillo & Artiodactlya & Camelidae & Palaeolama & major & Brazil & 0,13 & 0,00 & NA-SA \\
\hline 71274 & J. Alroy & Artiodactlya & Camelidae & Palaeolama & paradoxa & Uruguay & 0,78 & 0,01 & NA-SA \\
\hline 71325 & J. Alroy & Artiodactlya & Camelidae & Palaeolama & paradoxa & Uruguay & 0,78 & 0,01 & NA-SA \\
\hline 133634 & C. Jaramillo & Artiodactlya & Camelidae & Palaeolama & reissi & Ecuador & 2,59 & 0,01 & NA-SA \\
\hline 133644 & C. Jaramillo & Artiodactlya & Camelidae & Palaeolama & reissi & Ecuador & 2,59 & 0,01 & NA-SA \\
\hline 71310 & J. Alroy & Artiodactlya & Camelidae & Palaeolama & weddelli & Uruguay & 3,00 & 1,20 & NA-SA \\
\hline 142016 & C. Jaramillo & Artiodactlya & Camelidae & Palaeolama & weddeli & Bolivia & 2,59 & 0,78 & NA-SA \\
\hline 63515 & C. Bell & Artiodactlya & Camelidae & Palaeolama & sp. & Venezuela & 0,13 & 0,01 & NA-SA \\
\hline 70673 & D. Croft & Artiodactlya & Camelidae & Palaeolama & sp. & Bolivia & 2,59 & 0,78 & NA-SA \\
\hline 36611 & J. Alroy & Artiodactlya & Camelidae & Palaeolama & sp. & Argentina & 1,20 & 0,78 & NA-SA \\
\hline 70704 & D. Croft & Artiodactlya & Camelidae & Palaeolama & sp. & Bolivia & 0,78 & 0,13 & NA-SA \\
\hline 71265 & J. Alroy & Artiodactlya & Camelidae & Palaeolama & sp. & Chile & 0,13 & 0,01 & NA-SA \\
\hline 71292 & J. Alroy & Artiodactlya & Camelidae & Palaeolama & sp. & Paraguay & 2,59 & 0,01 & NA-SA \\
\hline 71295 & J. Alroy & Artiodactlya & Camelidae & Palaeolama & sp. & Brazil & 0,03 & 0,20 & NA-SA \\
\hline 71304 & J. Alroy & Artiodactlya & Camelidae & Palaeolama & sp. & Argentina & 2,59 & 0,01 & NA-SA \\
\hline 71322 & J. Alroy & Artiodactlya & Camelidae & Palaeolama & sp. & Bolivia & 1,20 & 0,78 & NA-SA \\
\hline 71323 & J. Alroy & Artiodactlya & Camelidae & Palaeolama & sp. & Bolivia & 1,20 & 0,78 & NA-SA \\
\hline 71335 & J. Alroy & Artiodactlya & Camelidae & Palaeolama & sp. & Brazil & 0,13 & 0,01 & NA-SA \\
\hline 133656 & C. Jaramillo & Artiodactlya & Camelidae & Palaeolama & sp. & Ecuador & 2,59 & 0,01 & NA-SA \\
\hline 142158 & C. Jaramillo & Artiodactlya & Camelidae & Palaeolama & sp. & Bolivia & 0,13 & 0,01 & NA-SA \\
\hline 142160 & C. Jaramillo & Artiodactlya & Camelidae & Palaeolama & sp. & Bolivia & 0,13 & 0,01 & NA-SA \\
\hline 144953 & C. Jaramillo & Artiodactlya & Camelidae & Palaeolama & sp. & Peru & 3,60 & 0,78 & NA-SA \\
\hline 144976 & C. Jaramillo & Artiodactlya & Camelidae & Palaeolama & sp. & Peru & 0,13 & 0,01 & NA-SA \\
\hline 145500 & C. Jaramillo & Artiodactlya & Camelidae & Palaeolama & sp. & Peru & 0,01 & 0,01 & NA-SA \\
\hline 145505 & C. Jaramillo & Artiodactlya & Camelidae & Palaeolama & sp. & Peru & 0,03 & 0,02 & NA-SA \\
\hline 71347 & J. Alroy & Artiodactlya & Camelidae & Vicugna & vicugna & Argentina & 0,78 & 0,01 & NA-SA \\
\hline 133633 & C. Jaramillo & Artiodactlya & Cervidae & Agalmaceros & blicki & Ecuador & 2,59 & 0,01 & NA-SA \\
\hline 133639 & C. Jaramillo & Artiodactlya & Cervidae & Agalmaceros & blicki & Ecuador & 2,59 & 0,01 & NA-SA \\
\hline 133644 & C. Jaramillo & Artiodactlya & Cervidae & Agalmaceros & blicki & Ecuador & 2,59 & 0,01 & NA-SA \\
\hline 133645 & C. Jaramillo & Artiodactlya & Cervidae & Agalmaceros & blicki & Ecuador & 2,59 & 0,01 & NA-SA \\
\hline 53926 & M. Uhen & Artiodactlya & Cervidae & Antifer & crassus & Argentina & 3,60 & 2,59 & NA-SA \\
\hline 71265 & J. Alroy & Artiodactlya & Cervidae & Antifer & niemeyeri & Chile & 0,13 & 0,01 & NA-SA \\
\hline 71266 & J. Alroy & Artiodactlya & Cervidae & Antifer & niemeyeri & Chile & 0,01 & 0,01 & NA-SA \\
\hline 71274 & J. Alroy & Artiodactlya & Cervidae & Antifer & ultra & Uruguay & 0,78 & 0,01 & NA-SA \\
\hline 71280 & J. Alroy & Artiodactlya & Cervidae & Antifer & sp. & Chile & 0,13 & 0,01 & NA-SA \\
\hline 71281 & J. Alroy & Artiodactlya & Cervidae & Antifer & sp. & Chile & 2,59 & 0,01 & NA-SA \\
\hline 148417 & C. Jaramillo & Artiodactlya & Cervidae & Antifer & sp. & Brazil & 0,13 & 0,01 & NA-SA \\
\hline Kerber et al. 2014 & M. Vallejo & Artiodactlya & Cervidae & Antifer & sp. & Brazil & 0,04 & 0,01 & NA-SA \\
\hline 71292 & J. Alroy & Artiodactlya & Cervidae & Blastocerus & dichotomus & Paraguay & 2,59 & 0,01 & NA-SA \\
\hline 71289 & J. Alroy & Artiodactlya & Cervidae & Blastocerus & sp. & Argentina & 0,78 & 0,01 & NA-SA \\
\hline 70673 & D. Croft & Artiodactlya & Cervidae & Charitoceros & tarijensis & Bolivia & 2,59 & 0,78 & NA-SA \\
\hline 140653 & C. Jaramillo & Artiodactlya & Cervidae & Epieurycerus & truncus & Argentina & 0,78 & 0,13 & NA-SA \\
\hline 70673 & D. Croft & Artiodactlya & Cervidae & Hippocamelus & sp. & Bolivia & 2,59 & 0,78 & NA-SA \\
\hline 71275 & J. Alroy & Artiodactlya & Cervidae & Hippocamelus & sp. & Brazil & 2,59 & 0,01 & NA-SA \\
\hline 71280 & J. Alroy & Artiodactlya & Cervidae & Hippocamelus & sp. & Chile & 0,13 & 0,01 & NA-SA \\
\hline 143054 & C. Jaramillo & Artiodactlya & Cervidae & Hippocamelus & sp. & Bolivia & 1,20 & 0,78 & NA-SA \\
\hline 71293 & J. Alroy & Artiodactlya & Cervidae & Mazama & americana & Brazil & 2,59 & 0,01 & NA-SA \\
\hline 71297 & J. Alroy & Artiodactlya & Cervidae & Mazama & americana & Brazil & 2,59 & 0,01 & NA-SA \\
\hline 71296 & J. Alroy & Artiodactlya & Cervidae & Mazama & andina & Peru & 0,13 & 0,01 & NA-SA \\
\hline 71313 & J. Alroy & Artiodactlya & Cervidae & Mazama & gouazoubira & Brazil & 0,02 & 0,02 & NA-SA \\
\hline 63515 & C. Bell & Artiodactlya & Cervidae & Mazama & sp. & Venezuela & 0,13 & 0,01 & NA-SA \\
\hline 71291 & J. Alroy & Artiodactlya & Cervidae & Mazama & sp. & Colombia & 0,78 & 0,01 & NA-SA \\
\hline 71295 & J. Alroy & Artiodactlya & Cervidae & Mazama & sp. & Brazil & 0,03 & 0,20 & NA-SA \\
\hline 71298 & J. Alroy & Artiodactlya & Cervidae & Mazama & sp. & Brazil & 2,59 & 0,01 & NA-SA \\
\hline 71301 & J. Alroy & Artiodactlya & Cervidae & Mazama & sp. & Brazil & 2,59 & 0,01 & NA-SA \\
\hline 71302 & J. Alroy & Artiodactlya & Cervidae & Mazama & sp. & Brazil & 2,59 & 0,01 & NA-SA \\
\hline 71332 & J. Alroy & Artiodactlya & Cervidae & Mazama & sp. & Brazil & 0,13 & 0,01 & NA-SA \\
\hline 71334 & J. Alroy & Artiodactlya & Cervidae & Mazama & sp. & Brazil & 0,13 & 0,01 & NA-SA \\
\hline 71335 & J. Alroy & Artiodactlya & Cervidae & Mazama & sp. & Brazil & 0,13 & 0,01 & NA-SA \\
\hline 92814 & J. Alroy & Artiodactlya & Cervidae & Mazama & sp. & Brazil & 2,59 & 0,00 & NA-SA \\
\hline 145500 & C. Jaramillo & Artiodactlya & Cervidae & Mazama & sp. & Peru & 0,01 & 0,01 & NA-SA \\
\hline 147815 & C. Jaramillo & Artiodactlya & Cervidae & Mazama & sp. & Brazil & 2,59 & 0,00 & NA-SA \\
\hline DeOliveira et al. 2014 & M. Vallejo & Artiodactlya & Cervidae & Mazama & sp. & Brazil & 0,01 & 0,01 & NA-SA \\
\hline 71303 & J. Alroy & Artiodactlya & Cervidae & Morenelaphus & azpeitianus & Argentina & 0,78 & 0,01 & NA-SA \\
\hline 71274 & J. Alroy & Artiodactlya & Cervidae & Morenelaphus & brachyceros & Uruguay & 0,78 & 0,01 & NA-SA \\
\hline 71274 & J. Alroy & Artiodactlya & Cervidae & Morenelaphus & lujanensis & Uruguay & 0,78 & 0,01 & NA-SA \\
\hline 71292 & J. Alroy & Artiodactlya & Cervidae & Morenelaphus & brachyceros & Paraguay & 2,59 & 0,01 & NA-SA \\
\hline 71303 & J. Alroy & Artiodactlya & Cervidae & Morenelaphus & lujanensis & Argentina & 0,78 & 0,01 & NA-SA \\
\hline 70705 & D. Croft & Artiodactlya & Cervidae & Morenelaphus & sp. & Bolivia & 0,01 & 0,00 & NA-SA \\
\hline 71316 & J. Alroy & Artiodactlya & Cervidae & Morenelaphus & sp. & Argentina & 0,13 & 0,01 & NA-SA \\
\hline 71324 & J. Alroy & Artiodactlya & Cervidae & Morenelaphus & sp. & Bolivia & 0,01 & 0,01 & NA-SA \\
\hline 71347 & J. Alroy & Artiodactlya & Cervidae & Morenelaphus & sp. & Argentina & 0,78 & 0,01 & NA-SA \\
\hline 78577 & J. Alroy & Artiodactlya & Cervidae & Morenelaphus & sp. & Argentina & 0,78 & 0,01 & NA-SA \\
\hline 114698 & M. Uhen & Artiodactlya & Cervidae & Morenelaphus & sp. & Argentina & 0,13 & 0,01 & NA-SA \\
\hline 148417 & C. Jaramillo & Artiodactlya & Cervidae & Morenelaphus & sp. & Brazil & 0,13 & 0,01 & NA-SA \\
\hline Kerber et al. 2014 & M. Vallejo & Artiodactlya & Cervidae & Morenelaphus & sp. & Brazil & 0,04 & 0,01 & NA-SA \\
\hline
\end{tabular}




\begin{tabular}{|c|c|c|c|c|c|c|c|c|c|}
\hline 133639 & C. Jaramillo & Artiodactlya & Cervidae & Odocoileus & peruvianus & Ecuador & 2,59 & 0,01 & NA-SA \\
\hline 133644 & C. Jaramillo & Artiodactlya & Cervidae & Odocoileus & peruvianus & Ecuador & 2,59 & 0,01 & NA-SA \\
\hline 133630 & C. Jaramillo & Artiodactlya & Cervidae & Odocoileus & salinae & Ecuador & 0,78 & 0,01 & NA-SA \\
\hline 146529 & C. Jaramillo & Artiodactlya & Cervidae & Odocoileus & salinae & Colombia & 2,59 & 0,01 & NA-SA \\
\hline Lindsey and Lopez 2014 & M. Vallejo & Artiodactlya & Cervidae & Odocoileus & salinae & Ecuador & 0,78 & 0,01 & NA-SA \\
\hline 144976 & C. Jaramillo & Artiodactlya & Cervidae & Odocoileus & sp. & Peru & 0,13 & 0,01 & NA-SA \\
\hline 145500 & C. Jaramillo & Artiodactlya & Cervidae & Odocoileus & sp. & Peru & 0,01 & 0,01 & NA-SA \\
\hline 63515 & C. Bell & Artiodactlya & Cervidae & Odocoileus & sp. & Venezuela & 0,13 & 0,01 & NA-SA \\
\hline 71346 & J. Alroy & Artiodactlya & Cervidae & Odocoileus & sp. & Peru & 0,13 & 0,01 & NA-SA \\
\hline 145505 & C. Jaramillo & Artiodactlya & Cervidae & Odocoileus & sp. & Peru & 0,03 & 0,02 & NA-SA \\
\hline 63338 & J. Alroy & Artiodactlya & Cervidae & Ozotoceros & bezoarticus & Argentina & 0,01 & 0,00 & NA-SA \\
\hline 63342 & D. Croft & Artiodactlya & Cervidae & Ozotoceros & bezoarticus & Argentina & 0,01 & 0,00 & NA-SA \\
\hline 71274 & J. Alroy & Artiodactlya & Cervidae & Ozotoceros & bezoarticus & Uruguay & 0,78 & 0,01 & NA-SA \\
\hline 71325 & J. Alroy & Artiodactlya & Cervidae & Ozotoceros & bezoarticus & Uruguay & 0,78 & 0,01 & NA-SA \\
\hline 73845 & J. Alroy & Artiodactlya & Cervidae & Ozotoceros & bezoarticus & Argentina & 0,13 & 0,01 & NA-SA \\
\hline 92814 & J. Alroy & Artiodactlya & Cervidae & Ozotoceros & sp. & Brazil & 2,59 & 0,00 & NA-SA \\
\hline Gonzalez et al. 2014 & M. Vallejo & Artiodactlya & Cervidae & Pudu & & Chile & 0,01 & 0,01 & NA-SA \\
\hline DeOliveira et al. 2014 & M. Vallejo & Artiodactlya & Cervidae & & & Brazil & 0,01 & 0,01 & NA-SA \\
\hline 142544 & C. Jaramillo & Artiodactlya & Tayassuidae & Catagonus & stenocephalus & Bolivia & 1,20 & 0,78 & NA-SA \\
\hline Kerber et al. 2014 & M. Vallejo & Artiodactlya & Tayassuidae & Catagonus & stenocephalus & Brazil & 0,04 & 0,01 & NA-SA \\
\hline 71274 & J. Alroy & Artiodactlya & Tayassuidae & Catagonus & sp. & Uruguay & 0,78 & 0,01 & NA-SA \\
\hline 71304 & J. Alroy & Artiodactlya & Tayassuidae & Catagonus & sp. & Argentina & 2,59 & 0,01 & NA-SA \\
\hline 93265 & J. Alroy & Artiodactlya & Tayassuidae & Catagonus & sp. & Argentina & 0,13 & 0,01 & NA-SA \\
\hline 148417 & C. Jaramillo & Artiodactlya & Tayassuidae & Catagonus & sp. & Brazil & 0,13 & 0,01 & NA-SA \\
\hline 71304 & J. Alroy & Artiodactlya & Tayassuidae & Platygonus & chapalmalensis & Argentina & 2,59 & 0,01 & NA-SA \\
\hline 70673 & D. Croft & Artiodactlya & Tayassuidae & Platygonus & tarijensis & Bolivia & 2,59 & 0,78 & NA-SA \\
\hline 141896 & C. Jaramillo & Artiodactlya & Tayassuidae & Platygonus & uquiensis & Argentina & 3,00 & 1,20 & NA-SA \\
\hline 140047 & C. Jaramillo & Artiodactlya & Tayassuidae & Platygonus & sp. & Uruguay & 5,33 & 0,78 & NA-SA \\
\hline 142544 & C. Jaramillo & Artiodactlya & Tayassuidae & Platygonus & sp. & Bolivia & 1,20 & 0,78 & NA-SA \\
\hline 133585 & C. Jaramillo & Artiodactlya & Tayassuidae & Selenogonus & narinoensis & Colombia & 3,60 & 0,01 & NA-SA \\
\hline 142016 & C. Jaramillo & Artiodactlya & Tayassuidae & Tayassu & major & Bolivia & 2,59 & 0,78 & NA-SA \\
\hline Kerber et al. 2014 & M. Vallejo & Artiodactlya & Tayassuidae & Tayassu & pecari & Brazil & 0,04 & 0,01 & NA-SA \\
\hline DeOliveira et al. 2014 & M. Vallejo & Artiodactlya & Tayassuidae & Tayassu & pecari & Brazil & 0,01 & 0,01 & NA-SA \\
\hline 71335 & J. Alroy & Artiodactlya & Tayassuidae & Tayassu & pecari & Brazil & 0,13 & 0,01 & NA-SA \\
\hline 71347 & J. Alroy & Artiodactlya & Tayassuidae & Tayassu & pecari & Argentina & 0,78 & 0,01 & NA-SA \\
\hline 71291 & J. Alroy & Artiodactlya & Tayassuidae & Tayassu & pecari & Colombia & 0,78 & 0,01 & NA-SA \\
\hline 71295 & J. Alroy & Artiodactlya & Tayassuidae & Tayassu & pecari & Brazil & 0,03 & 0,20 & NA-SA \\
\hline 71332 & J. Alroy & Artiodactlya & Tayassuidae & Tayassu & pecari & Brazil & 0,13 & 0,01 & NA-SA \\
\hline 71334 & J. Alroy & Artiodactlya & Tayassuidae & Tayassu & pecari & Brazil & 0,13 & 0,01 & NA-SA \\
\hline 71303 & J. Alroy & Artiodactlya & Tayassuidae & Tayassu & tajacu & Argentina & 0,78 & 0,01 & NA-SA \\
\hline 71313 & J. Alroy & Artiodactlya & Tayassuidae & Tayassu & tajacu & Brazil & 0,02 & 0,02 & NA-SA \\
\hline 71314 & J. Alroy & Artiodactlya & Tayassuidae & Tayassu & tajacu & Argentina & 0,78 & 0,01 & NA-SA \\
\hline 71291 & J. Alroy & Artiodactlya & Tayassuidae & Tayassu & tajacu & Colombia & 0,78 & 0,01 & NA-SA \\
\hline 71332 & J. Alroy & Artiodactlya & Tayassuidae & Tayassu & tajacu & Brazil & 0,13 & 0,01 & NA-SA \\
\hline 71334 & J. Alroy & Artiodactlya & Tayassuidae & Tayassu & tajacu & Brazil & 0,13 & 0,01 & NA-SA \\
\hline 71335 & J. Alroy & Artiodactlya & Tayassuidae & Tayassu & tajacu & Brazil & 0,13 & 0,01 & NA-SA \\
\hline 71285 & J. Alroy & Artiodactlya & Tayassuidae & Tayassu & tajacu & Colombia & 0,78 & 0,01 & NA-SA \\
\hline 92814 & J. Alroy & Artiodactlya & Tayassuidae & Tayassu & sp. & Brazil & 2,59 & 0,00 & NA-SA \\
\hline 71301 & J. Alroy & Artiodactlya & Tayassuidae & Tayassu & sp. & Brazil & 2,59 & 0,01 & NA-SA \\
\hline 71302 & J. Alroy & Artiodactlya & Tayassuidae & Tayassu & sp. & Brazil & 2,59 & 0,01 & NA-SA \\
\hline 63335 & D. Croft & Artiodactlya & Tayassuidae & Tayassu & sp. & Argentina & 0,78 & 0,13 & NA-SA \\
\hline 93267 & J. Alroy & Artiodactlya & Tayassuidae & Tayassu & sp. & Argentina & 0,01 & 0,01 & NA-SA \\
\hline 73845 & J. Alroy & Carnivora & Canidae & Canis & avus & Argentina & 0,13 & 0,01 & NA-SA \\
\hline 70673 & D. Croft & Carnivora & Canidae & Canis & dirus & Bolivia & 2,59 & 0,78 & NA-SA \\
\hline 144976 & C. Jaramillo & Carnivora & Canidae & Canis & dirus & Peru & 0,13 & 0,01 & NA-SA \\
\hline 71346 & J. Alroy & Carnivora & Canidae & Canis & dirus & Peru & 0,13 & 0,01 & NA-SA \\
\hline 63515 & C. Bell & Carnivora & Canidae & Canis & dirus & Venezuela & 0,13 & 0,01 & NA-SA \\
\hline 145366 & C. Jaramillo & Carnivora & Canidae & Canis & dirus & Venezuela & 0,13 & 0,01 & NA-SA \\
\hline 71329 & J. Alroy & Carnivora & Canidae & Canis & familiaris & Peru & 0,01 & 0,01 & NA-SA \\
\hline 71303 & J. Alroy & Carnivora & Canidae & Canis & griseus & Argentina & 0,78 & 0,01 & NA-SA \\
\hline 63338 & J. Alroy & Carnivora & Canidae & Canis & gymnocercus & Argentina & 0,01 & 0,00 & NA-SA \\
\hline 70673 & D. Croft & Carnivora & Canidae & Canis & gymnocercus & Bolivia & 2,59 & 0,78 & NA-SA \\
\hline 71286 & J. Alroy & Carnivora & Canidae & Canis & gymnocercus & Argentina & 0,78 & 0,01 & NA-SA \\
\hline 71303 & J. Alroy & Carnivora & Canidae & Canis & gymnocercus & Argentina & 0,78 & 0,01 & NA-SA \\
\hline 142016 & C. Jaramillo & Carnivora & Canidae & Canis & proplatensis & Bolivia & 2,59 & 0,78 & NA-SA \\
\hline 70704 & D. Croft & Carnivora & Canidae & Canis & sp. & Bolivia & 0,78 & 0,13 & NA-SA \\
\hline 71338 & J. Alroy & Carnivora & Canidae & Canis & sp. & Argentina & 0,13 & 0,01 & NA-SA \\
\hline 71342 & J. Alroy & Carnivora & Canidae & Canis & sp. & Chile & 0,13 & 0,01 & NA-SA \\
\hline 71304 & J. Alroy & Carnivora & Canidae & Canis & sp. & Argentina & 2,59 & 0,01 & NA-SA \\
\hline 71316 & J. Alroy & Carnivora & Canidae & Canis & sp. & Argentina & 0,13 & 0,01 & NA-SA \\
\hline 71343 & J. Alroy & Carnivora & Canidae & Canis & sp. & Argentina & 0,01 & 0,01 & NA-SA \\
\hline 71346 & J. Alroy & Carnivora & Canidae & Canis & sp. & Peru & 0,13 & 0,01 & NA-SA \\
\hline 63335 & D. Croft & Carnivora & Canidae & Canis & sp. & Argentina & 0,78 & 0,13 & NA-SA \\
\hline 145500 & C. Jaramillo & Carnivora & Canidae & Canis & sp. & Peru & 0,01 & 0,01 & NA-SA \\
\hline 145505 & C. Jaramillo & Carnivora & Canidae & Canis & sp. & Peru & 0,03 & 0,02 & NA-SA \\
\hline 146602 & C. Jaramillo & Carnivora & Canidae & Canis & sp. & Peru & 0,02 & 0,01 & NA-SA \\
\hline 71313 & J. Alroy & Carnivora & Canidae & Cerdocyon & thous & Brazil & 0,02 & 0,02 & NA-SA \\
\hline 148309 & C. Jaramillo & Carnivora & Canidae & Cerdocyon & thous & Brazil & 0,13 & 0,00 & NA-SA \\
\hline 70673 & D. Croft & Carnivora & Canidae & Chrysocyon & brachyurus & Bolivia & 2,59 & 0,78 & NA-SA \\
\hline
\end{tabular}




\begin{tabular}{|c|c|c|c|c|c|c|c|c|c|}
\hline 92814 & J. Alroy & Carnivora & Canidae & Chrysocyon & brachyurus & Brazil & 2,59 & 0,00 & NA-SA \\
\hline 92814 & J. Alroy & Carnivora & Canidae & Chrysocyon & sp. & Brazil & 2,59 & 0,00 & NA-SA \\
\hline 59978 & M. Uhen & Carnivora & Canidae & Dusicyon & avus & Brazil & 0,13 & 0,01 & NA-SA \\
\hline 71336 & J. Alroy & Carnivora & Canidae & Dusicyon & avus & Argentina & 0,13 & 0,01 & NA-SA \\
\hline 71341 & J. Alroy & Carnivora & Canidae & Dusicyon & avus & Chile & 0,01 & 0,01 & NA-SA \\
\hline 71342 & J. Alroy & Carnivora & Canidae & Dusicyon & avus & Chile & 0,13 & 0,01 & NA-SA \\
\hline 71286 & J. Alroy & Carnivora & Canidae & Dusicyon & avus & Argentina & 0,78 & 0,01 & NA-SA \\
\hline 140159 & C. Jaramillo & Carnivora & Canidae & Dusicyon & avus & Uruguay & 0,12 & 0,04 & NA-SA \\
\hline 71303 & J. Alroy & Carnivora & Canidae & Dusicyon & avus & Argentina & 0,78 & 0,01 & NA-SA \\
\hline 133644 & C. Jaramillo & Carnivora & Canidae & Dusicyon & culpaeus & Ecuador & 2,59 & 0,01 & NA-SA \\
\hline 70705 & D. Croft & Carnivora & Canidae & Dusicyon & griseus & Bolivia & 0,01 & 0,00 & NA-SA \\
\hline 133630 & C. Jaramillo & Carnivora & Canidae & Dusicyon & sechurae & Ecuador & 0,78 & 0,01 & NA-SA \\
\hline 71265 & J. Alroy & Carnivora & Canidae & Dusicyon & sp. & Chile & 0,13 & 0,01 & NA-SA \\
\hline 93267 & J. Alroy & Carnivora & Canidae & Dusicyon & sp. & Argentina & 0,01 & 0,01 & NA-SA \\
\hline 123831 & M. Uhen & Carnivora & Canidae & Dusicyon & sp. & Argentina & 2,59 & 0,01 & NA-SA \\
\hline 71304 & J. Alroy & Carnivora & Canidae & Dusicyon & sp. & Argentina & 2,59 & 0,01 & NA-SA \\
\hline 71324 & J. Alroy & Carnivora & Canidae & Dusicyon & sp. & Bolivia & 0,01 & 0,01 & NA-SA \\
\hline 144976 & C. Jaramillo & Carnivora & Canidae & Dusicyon & sp. & Peru & 0,13 & 0,01 & NA-SA \\
\hline Ramirez and Prevosti 2014 & M. Vallejo & Carnivora & Canidae & Lycalopex & ensenadensis & Argentina & 0,03 & 0,03 & NA-SA \\
\hline 133630 & C. Jaramillo & Carnivora & Canidae & Protocyon & orcesi & Ecuador & 0,78 & 0,01 & NA-SA \\
\hline 71304 & J. Alroy & Carnivora & Canidae & Protocyon & scagliaruen & Argentina & 2,59 & 0,01 & NA-SA \\
\hline 142016 & C. Jaramillo & Carnivora & Canidae & Protocyon & tarijensis & Bolivia & 2,59 & 0,78 & NA-SA \\
\hline 63515 & C. Bell & Carnivora & Canidae & Protocyon & troglodytes & Venezuela & 0,13 & 0,01 & NA-SA \\
\hline 70704 & D. Croft & Carnivora & Canidae & Protocyon & troglodytes & Bolivia & 0,78 & 0,13 & NA-SA \\
\hline 71313 & J. Alroy & Carnivora & Canidae & Protocyon & troglodytes & Brazil & 0,02 & 0,02 & NA-SA \\
\hline 71332 & J. Alroy & Carnivora & Canidae & Protocyon & troglodytes & Brazil & 0,13 & 0,01 & NA-SA \\
\hline 92814 & J. Alroy & Carnivora & Canidae & Protocyon & troglodytes & Brazil & 2,59 & 0,00 & NA-SA \\
\hline 140159 & C. Jaramillo & Carnivora & Canidae & Protocyon & troglodytes & Uruguay & 0,12 & 0,04 & NA-SA \\
\hline 145366 & C. Jaramillo & Carnivora & Canidae & Protocyon & troglodytes & Venezuela & 0,13 & 0,01 & NA-SA \\
\hline 148309 & C. Jaramillo & Carnivora & Canidae & Protocyon & troglodytes & Brazil & 0,13 & 0,00 & NA-SA \\
\hline 145366 & C. Jaramillo & Carnivora & Canidae & Protocyon & sp. & Venezuela & 0,13 & 0,01 & NA-SA \\
\hline 71298 & J. Alroy & Carnivora & Canidae & Protocyon & sp. & Brazil & 2,59 & 0,01 & NA-SA \\
\hline 63515 & C. Bell & Carnivora & Canidae & Protocyon & sp. & Venezuela & 0,13 & 0,01 & NA-SA \\
\hline 71301 & J. Alroy & Carnivora & Canidae & Protocyon & sp. & Brazil & 2,59 & 0,01 & NA-SA \\
\hline 71302 & J. Alroy & Carnivora & Canidae & Protocyon & sp. & Brazil & 2,59 & 0,01 & NA-SA \\
\hline 71322 & J. Alroy & Carnivora & Canidae & Protocyon & sp. & Bolivia & 1,20 & 0,78 & NA-SA \\
\hline 71302 & J. Alroy & Carnivora & Canidae & Speothos & sp. & Brazil & 2,59 & 0,01 & NA-SA \\
\hline 70673 & D. Croft & Carnivora & Canidae & Theriodictis & tarijensis & Bolivia & 2,59 & 0,78 & NA-SA \\
\hline 59978 & M. Uhen & Carnivora & Canidae & Theriodictis & sp. & Brazil & 0,13 & 0,01 & NA-SA \\
\hline 71288 & J. Alroy & Carnivora & Canidae & Theriodictis & sp. & Argentina & 1,20 & 0,01 & NA-SA \\
\hline 63515 & C. Bell & Carnivora & Canidae & Urocyon & cinereoargenteus & Venezuela & 0,13 & 0,01 & NA-SA \\
\hline 145366 & C. Jaramillo & Carnivora & Canidae & Urocyon & cinereoargenteus & Venezuela & 0,13 & 0,01 & NA-SA \\
\hline 71338 & J. Alroy & Carnivora & Felidae & Felis & listai & Argentina & 0,13 & 0,01 & NA-SA \\
\hline 71341 & J. Alroy & Carnivora & Felidae & Felis & listai & Chile & 0,01 & 0,01 & NA-SA \\
\hline 71344 & J. Alroy & Carnivora & Felidae & Felis & listai & Chile & 0,13 & 0,01 & NA-SA \\
\hline 133644 & C. Jaramillo & Carnivora & Felidae & Felis & platensis & Ecuador & 2,59 & 0,01 & NA-SA \\
\hline 142016 & C. Jaramillo & Carnivora & Felidae & Felis & platensis & Bolivia & 2,59 & 0,78 & NA-SA \\
\hline 70673 & D. Croft & Carnivora & Felidae & Felis & yaguaroundi & Bolivia & 2,59 & 0,78 & NA-SA \\
\hline 71302 & J. Alroy & Carnivora & Felidae & Felis & sp. & Brazil & 2,59 & 0,01 & NA-SA \\
\hline 63335 & D. Croft & Carnivora & Felidae & Herpailurus & yaguaroundi & Argentina & 0,78 & 0,13 & NA-SA \\
\hline 144977 & C. Jaramillo & Carnivora & Felidae & Homotherium & venezuelensis & Venezuela & 1,00 & 0,50 & NA-SA \\
\hline 92814 & J. Alroy & Carnivora & Felidae & Leopardus & braccatus & Brazil & 2,59 & 0,00 & NA-SA \\
\hline 71313 & J. Alroy & Carnivora & Felidae & Leopardus & pardalis & Brazil & 0,02 & 0,02 & NA-SA \\
\hline 136420 & C. Jaramillo & Carnivora & Felidae & Leopardus & pardalis & Brazil & 0,13 & 0,00 & NA-SA \\
\hline 136498 & C. Jaramillo & Carnivora & Felidae & Leopardus & pardalis & Brazil & 0,13 & 0,00 & NA-SA \\
\hline 71313 & J. Alroy & Carnivora & Felidae & Leopardus & tigrina & Brazil & 0,02 & 0,02 & NA-SA \\
\hline 148309 & C. Jaramillo & Carnivora & Felidae & Leopardus & tigrinus & Brazil & 0,13 & 0,00 & NA-SA \\
\hline 13907 & J. Alroy & Carnivora & Felidae & Leopardus & vorohuensis & Argentina & 3,00 & 1,20 & NA-SA \\
\hline 71346 & J. Alroy & Carnivora & Felidae & Panthera & leo & Peru & 0,13 & 0,01 & NA-SA \\
\hline 144976 & C. Jaramillo & Carnivora & Felidae & Panthera & leo & Peru & 0,13 & 0,01 & NA-SA \\
\hline 70673 & D. Croft & Carnivora & Felidae & Panthera & onca & Bolivia & 2,59 & 0,78 & NA-SA \\
\hline 70704 & D. Croft & Carnivora & Felidae & Panthera & onca & Bolivia & 0,78 & 0,13 & NA-SA \\
\hline 71303 & J. Alroy & Carnivora & Felidae & Panthera & onca & Argentina & 0,78 & 0,01 & NA-SA \\
\hline 71313 & J. Alroy & Carnivora & Felidae & Panthera & onca & Brazil & 0,02 & 0,02 & NA-SA \\
\hline 71322 & J. Alroy & Carnivora & Felidae & Panthera & onca & Bolivia & 1,20 & 0,78 & NA-SA \\
\hline 71335 & J. Alroy & Carnivora & Felidae & Panthera & onca & Brazil & 0,13 & 0,01 & NA-SA \\
\hline 71341 & J. Alroy & Carnivora & Felidae & Panthera & onca & Chile & 0,01 & 0,01 & NA-SA \\
\hline 71587 & D. Croft & Carnivora & Felidae & Panthera & onca & Bolivia & 2,59 & 0,01 & NA-SA \\
\hline 92814 & J. Alroy & Carnivora & Felidae & Panthera & onca & Brazil & 2,59 & 0,00 & NA-SA \\
\hline 133644 & C. Jaramillo & Carnivora & Felidae & Panthera & onca & Ecuador & 2,59 & 0,01 & NA-SA \\
\hline 136498 & C. Jaramillo & Carnivora & Felidae & Panthera & onca & Brazil & 0,13 & 0,00 & NA-SA \\
\hline 141367 & C. Jaramillo & Carnivora & Felidae & Panthera & onca & Chile & 0,13 & 0,01 & NA-SA \\
\hline 145508 & C. Jaramillo & Carnivora & Felidae & Panthera & onca & Peru & 2,59 & 0,01 & NA-SA \\
\hline 147609 & C. Jaramillo & Carnivora & Felidae & Panthera & onca & Brazil & 2,59 & 0,00 & NA-SA \\
\hline 147815 & C. Jaramillo & Carnivora & Felidae & Panthera & onca & Brazil & 2,59 & 0,00 & NA-SA \\
\hline 71303 & J. Alroy & Carnivora & Felidae & Panthera & platensis & Argentina & 0,78 & 0,01 & NA-SA \\
\hline 145500 & C. Jaramillo & Carnivora & Felidae & Panthera & sp. & Peru & 0,01 & 0,01 & NA-SA \\
\hline 70673 & D. Croft & Carnivora & Felidae & Puma & concolor & Bolivia & 2,59 & 0,78 & NA-SA \\
\hline 71274 & J. Alroy & Carnivora & Felidae & Puma & concolor & Uruguay & 0,78 & 0,01 & NA-SA \\
\hline
\end{tabular}




\begin{tabular}{|c|c|c|c|c|c|c|c|c|c|}
\hline 71313 & J. Alroy & Carnivora & Felidae & Puma & concolor & Brazil & 0,02 & 0,02 & NA-SA \\
\hline 73845 & J. Alroy & Carnivora & Felidae & Puma & concolor & Argentina & 0,13 & 0,01 & NA-SA \\
\hline 136498 & C. Jaramillo & Carnivora & Felidae & Puma & concolor & Brazil & 0,13 & 0,00 & NA-SA \\
\hline 141367 & C. Jaramillo & Carnivora & Felidae & Puma & concolor & Chile & 0,13 & 0,01 & NA-SA \\
\hline 71313 & J. Alroy & Carnivora & Felidae & Puma & yagouaroundi & Brazil & 0,02 & 0,02 & NA-SA \\
\hline 71332 & J. Alroy & Carnivora & Felidae & Puma & yagouaroundi & Brazil & 0,13 & 0,01 & NA-SA \\
\hline 71335 & J. Alroy & Carnivora & Felidae & Puma & yagouaroundi & Brazil & 0,13 & 0,01 & NA-SA \\
\hline 145576 & C. Jaramillo & Carnivora & Felidae & Puma & sp. & Peru & 2,59 & 0,01 & NA-SA \\
\hline 147999 & C. Jaramillo & Carnivora & Felidae & Smilodon & bonaerensis & Argentina & 1,20 & 0,78 & NA-SA \\
\hline 147999 & C. Jaramillo & Carnivora & Felidae & Smilodon & ensenadenis & Argentina & 1,20 & 0,78 & NA-SA \\
\hline 71346 & J. Alroy & Carnivora & Felidae & Smilodon & fatalis & Peru & 0,13 & 0,01 & NA-SA \\
\hline 144977 & C. Jaramillo & Carnivora & Felidae & Smilodon & gracilis & Venezuela & 1,00 & 0,50 & NA-SA \\
\hline 59978 & M. Uhen & Carnivora & Felidae & Smilodon & populator & Brazil & 0,13 & 0,01 & NA-SA \\
\hline 63515 & C. Bell & Carnivora & Felidae & Smilodon & populator & Venezuela & 0,13 & 0,01 & NA-SA \\
\hline 63884 & C. Bell & Carnivora & Felidae & Smilodon & populator & Venezuela & 2,59 & 0,01 & NA-SA \\
\hline 70673 & D. Croft & Carnivora & Felidae & Smilodon & populator & Bolivia & 2,59 & 0,78 & NA-SA \\
\hline 70704 & D. Croft & Carnivora & Felidae & Smilodon & populator & Bolivia & 0,78 & 0,13 & NA-SA \\
\hline 71287 & J. Alroy & Carnivora & Felidae & Smilodon & populator & Argentina & 0,78 & 0,01 & NA-SA \\
\hline 71303 & J. Alroy & Carnivora & Felidae & Smilodon & populator & Argentina & 0,78 & 0,01 & NA-SA \\
\hline 71313 & J. Alroy & Carnivora & Felidae & Smilodon & populator & Brazil & 0,02 & 0,02 & NA-SA \\
\hline 71332 & J. Alroy & Carnivora & Felidae & Smilodon & populator & Brazil & 0,13 & 0,01 & NA-SA \\
\hline 71334 & J. Alroy & Carnivora & Felidae & Smilodon & populator & Brazil & 0,13 & 0,01 & NA-SA \\
\hline 71335 & J. Alroy & Carnivora & Felidae & Smilodon & populator & Brazil & 0,13 & 0,01 & NA-SA \\
\hline 78577 & J. Alroy & Carnivora & Felidae & Smilodon & populator & Argentina & 0,78 & 0,01 & NA-SA \\
\hline 92814 & J. Alroy & Carnivora & Felidae & Smilodon & populator & Brazil & 2,59 & 0,00 & NA-SA \\
\hline 93235 & M. Uhen & Carnivora & Felidae & Smilodon & populator & Brazil & 0,13 & 0,00 & NA-SA \\
\hline 123831 & M. Uhen & Carnivora & Felidae & Smilodon & populator & Argentina & 2,59 & 0,01 & NA-SA \\
\hline 136498 & C. Jaramillo & Carnivora & Felidae & Smilodon & populator & Brazil & 0,13 & 0,00 & NA-SA \\
\hline 136499 & C. Jaramillo & Carnivora & Felidae & Smilodon & populator & Brazil & 0,13 & 0,00 & NA-SA \\
\hline 142016 & C. Jaramillo & Carnivora & Felidae & Smilodon & populator & Bolivia & 2,59 & 0,78 & NA-SA \\
\hline 147609 & C. Jaramillo & Carnivora & Felidae & Smilodon & populator & Brazil & 2,59 & 0,00 & NA-SA \\
\hline 148309 & C. Jaramillo & Carnivora & Felidae & Smilodon & populator & Brazil & 0,13 & 0,00 & NA-SA \\
\hline 148397 & C. Jaramillo & Carnivora & Felidae & Smilodon & populator & Brazil & 0,01 & 0,00 & NA-SA \\
\hline 71274 & J. Alroy & Carnivora & Felidae & Smilodon & sp. & Uruguay & 0,78 & 0,01 & NA-SA \\
\hline 71298 & J. Alroy & Carnivora & Felidae & Smilodon & sp. & Brazil & 2,59 & 0,01 & NA-SA \\
\hline 71301 & J. Alroy & Carnivora & Felidae & Smilodon & sp. & Brazil & 2,59 & 0,01 & NA-SA \\
\hline 71322 & J. Alroy & Carnivora & Felidae & Smilodon & sp. & Bolivia & 1,20 & 0,78 & NA-SA \\
\hline 133630 & C. Jaramillo & Carnivora & Felidae & Smilodon & sp. & Ecuador & 0,78 & 0,01 & NA-SA \\
\hline 133644 & C. Jaramillo & Carnivora & Felidae & Smilodon & sp. & Ecuador & 2,59 & 0,01 & NA-SA \\
\hline 142158 & C. Jaramillo & Carnivora & Felidae & Smilodon & sp. & Bolivia & 0,13 & 0,01 & NA-SA \\
\hline 144976 & C. Jaramillo & Carnivora & Felidae & Smilodon & sp. & Peru & 0,13 & 0,01 & NA-SA \\
\hline 145500 & C. Jaramillo & Carnivora & Felidae & Smilodon & sp. & Peru & 0,01 & 0,01 & NA-SA \\
\hline 147999 & C. Jaramillo & Carnivora & Felidae & Smilodontidion & riggi & Argentina & 1,20 & 0,78 & NA-SA \\
\hline 71304 & J. Alroy & Carnivora & Felidae & Smilodontidion & riggii & Argentina & 2,59 & 0,01 & NA-SA \\
\hline 147999 & C. Jaramillo & Carnivora & Felidae & Smilodontidion & sp. & Argentina & 1,20 & 0,78 & NA-SA \\
\hline 71326 & J. Alroy & Carnivora & Mustelidae & Conepatus & rex & Peru & 0,01 & 0,00 & NA-SA \\
\hline 71327 & J. Alroy & Carnivora & Mustelidae & Conepatus & rex & Peru & 0,01 & 0,01 & NA-SA \\
\hline 71328 & J. Alroy & Carnivora & Mustelidae & Conepatus & rex & Peru & 0,01 & 0,01 & NA-SA \\
\hline 71329 & J. Alroy & Carnivora & Mustelidae & Conepatus & rex & Peru & 0,01 & 0,01 & NA-SA \\
\hline 71313 & J. Alroy & Carnivora & Mustelidae & Conepatus & semistriatus & Brazil & 0,02 & 0,02 & NA-SA \\
\hline 136498 & C. Jaramillo & Carnivora & Mustelidae & Conepatus & semistriatus & Brazil & 0,13 & 0,00 & NA-SA \\
\hline 136500 & C. Jaramillo & Carnivora & Mustelidae & Conepatus & semistriatus & Brazil & 0,13 & 0,00 & NA-SA \\
\hline 70673 & D. Croft & Carnivora & Mustelidae & Conepatus & suffocans & Bolivia & 2,59 & 0,78 & NA-SA \\
\hline 142016 & C. Jaramillo & Carnivora & Mustelidae & Conepatus & suffocans & Bolivia & 2,59 & 0,78 & NA-SA \\
\hline 145500 & C. Jaramillo & Carnivora & Mustelidae & Conepatus & sp. & Peru & 0,01 & 0,01 & NA-SA \\
\hline 146602 & C. Jaramillo & Carnivora & Mustelidae & Conepatus & sp. & Peru & 0,02 & 0,01 & NA-SA \\
\hline Pino et al. 2013 & M. Vallejo & Carnivora & Mustelidae & Conepatus & sp. & Chile & 0,01 & 0,01 & NA-SA \\
\hline 136420 & C. Jaramillo & Carnivora & Mustelidae & Eira & barbara & Brazil & 0,13 & 0,00 & NA-SA \\
\hline Castro et al. 2014 & M. Vallejo & Carnivora & Mustelidae & Eira & barbara & Brazil & 0,13 & 0,01 & NA-SA \\
\hline 71304 & J. Alroy & Carnivora & Mustelidae & Galictis & sorgentini & Argentina & 2,59 & 0,01 & NA-SA \\
\hline 71303 & J. Alroy & Carnivora & Mustelidae & Lontra & longicaudis & Argentina & 0,78 & 0,01 & NA-SA \\
\hline 136498 & C. Jaramillo & Carnivora & Mustelidae & Lontra & longicaudis & Brazil & 0,13 & 0,00 & NA-SA \\
\hline 71303 & J. Alroy & Carnivora & Mustelidae & Lyncodon & patagonicus & Argentina & 0,78 & 0,01 & NA-SA \\
\hline 92299 & M. Uhen & Carnivora & Mustelidae & Pteronura & brasiliensis & Argentina & 0,13 & 0,01 & NA-SA \\
\hline 92815 & J. Alroy & Carnivora & Mustelidae & Pteronura & brasiliensis & Brazil & 2,59 & 0,00 & NA-SA \\
\hline 136521 & C. Jaramillo & Carnivora & Mustelidae & Pteronura & brasiliensis & Brazil & 0,13 & 0,01 & NA-SA \\
\hline Forasiepi et al. 2014 & M. Vallejo & Carnivora & Procyonidae & Chapalmalania & sp. & Colombia & 3,60 & 3,60 & NA-SA \\
\hline 55600 & M. Uhen & Carnivora & Procyonidae & Cyonasua & argentina & Argentina & 9,00 & 6,80 & NA-SA \\
\hline 87198 & J. Alroy & Carnivora & Procyonidae & Cyonasua & prebrevirostris & Argentina & 9,00 & 6,80 & NA-SA \\
\hline 87198 & J. Alroy & Carnivora & Procyonidae & Cyonasua & brevirostris & Argentina & 9,00 & 6,80 & NA-SA \\
\hline 140630 & C. Jaramillo & Carnivora & Procyonidae & Cyonasua & groeberi & Argentina & 9,00 & 4,00 & NA-SA \\
\hline 140712 & C. Jaramillo & Carnivora & Procyonidae & Cyonasua & pascuali & Argentina & 9,00 & 6,80 & NA-SA \\
\hline 152061 & C. Jaramillo & Carnivora & Procyonidae & Cyonasua & sp. & Argentina & 9,00 & 6,80 & NA-SA \\
\hline Forasiepi et al. 2014 & M. Vallejo & Carnivora & Procyonidae & Cyonasua & sp. & Venezuela & 5,30 & 2,70 & NA-SA \\
\hline 70673 & D. Croft & Carnivora & Procyonidae & Nasua & sp. & Bolivia & 2,59 & 0,78 & NA-SA \\
\hline 13503 & J. Alroy & Carnivora & Procyonidae & Parahyaenodon & argentinus & Argentina & 6,80 & 4,00 & NA-SA \\
\hline 71313 & J. Alroy & Carnivora & Procyonidae & Procyon & cancrivorus & Brazil & 0,02 & 0,02 & NA-SA \\
\hline 136420 & C. Jaramillo & Carnivora & Procyonidae & Procyon & cancrivorus & Brazil & 0,13 & 0,00 & NA-SA \\
\hline 70673 & D. Croft & Carnivora & Ursidae & Arctodus & tarijensis & Bolivia & 2,59 & 0,78 & NA-SA \\
\hline
\end{tabular}




\begin{tabular}{|c|c|c|c|c|c|c|c|c|c|}
\hline 70673 & D. Croft & Carnivora & Ursidae & Arctodus & wingei & Bolivia & 2,59 & 0,78 & NA-SA \\
\hline 71335 & J. Alroy & Carnivora & Ursidae & Arctotherium & bonaerense & Brazil & 0,13 & 0,01 & NA-SA \\
\hline 71313 & J. Alroy & Carnivora & Ursidae & Arctotherium & brasiliense & Brazil & 0,02 & 0,02 & NA-SA \\
\hline 71334 & J. Alroy & Carnivora & Ursidae & Arctotherium & brasiliense & Brazil & 0,13 & 0,01 & NA-SA \\
\hline 107828 & J. Alroy & Carnivora & Ursidae & Arctotherium & brasiliense & Brazil & 2,59 & 0,01 & NA-SA \\
\hline 142016 & C. Jaramillo & Carnivora & Ursidae & Arctotherium & tarijense & Bolivia & 2,59 & 0,78 & NA-SA \\
\hline 142016 & C. Jaramillo & Carnivora & Ursidae & Arctotherium & wingei & Bolivia & 2,59 & 0,78 & NA-SA \\
\hline 145376 & C. Jaramillo & Carnivora & Ursidae & Arctotherium & wingei & Venezuela & 0,13 & 0,01 & NA-SA \\
\hline 145377 & C. Jaramillo & Carnivora & Ursidae & Arctotherium & wingei & Venezuela & 0,13 & 0,00 & NA-SA \\
\hline 148309 & C. Jaramillo & Carnivora & Ursidae & Arctotherium & sp. & Brazil & 0,13 & 0,00 & NA-SA \\
\hline 70704 & D. Croft & Carnivora & Ursidae & Arctotherium & sp. & Bolivia & 0,78 & 0,13 & NA-SA \\
\hline 71283 & J. Alroy & Carnivora & Ursidae & Arctotherium & sp. & Venezuela & 0,01 & 0,01 & NA-SA \\
\hline 71303 & J. Alroy & Carnivora & Ursidae & Arctotherium & sp. & Argentina & 0,78 & 0,01 & NA-SA \\
\hline 141367 & C. Jaramillo & Carnivora & Ursidae & Arctotherium & sp. & Chile & 0,13 & 0,01 & NA-SA \\
\hline 71322 & J. Alroy & Carnivora & Ursidae & Arctotherium & sp. & Bolivia & 1,20 & 0,78 & NA-SA \\
\hline Salles et al. 2014 & M. Vallejo & Chiroptera & Furipteridae & Furipterus & horrens & Brazil & 0,09 & 0,09 & NA-SA \\
\hline 74292 & J. Alroy & Chiroptera & Molossidae & Eumops & glaucinus & United States & 0,13 & 0,01 & SA-NA \\
\hline 19638 & J. Alroy & Chiroptera & Molossidae & Eumops & perotis & United States & 4,90 & 1,80 & SA-NA \\
\hline 63724 & C. Bell & Chiroptera & Molossidae & Eumops & perotis & United States & 0,01 & 0,00 & SA-NA \\
\hline 71264 & J. Alroy & Chiroptera & Molossidae & Eumops & perotis & Mexico & 0,13 & 0,01 & SA-NA \\
\hline 71313 & J. Alroy & Chiroptera & Molossidae & Tadarida & brasiliensis & Brazil & 0,02 & 0,02 & NA-SA \\
\hline 71313 & J. Alroy & Chiroptera & Mormoopidae & Mormoops & megalophylla & Brazil & 0,02 & 0,02 & NA-SA \\
\hline Salles et al. 2014 & M. Vallejo & Chiroptera & Mormoopidae & Mormoops & megalophylla & Brazil & 0,09 & 0,09 & NA-SA \\
\hline Salles et al. 2014 & M. Vallejo & Chiroptera & Mormoopidae & Pteronotus & gymnonotus & Brazil & 0,09 & 0,09 & NA-SA \\
\hline 71313 & J. Alroy & Chiroptera & Mormoopidae & Pteronotus & parnelli & Brazil & 0,02 & 0,02 & NA-SA \\
\hline Salles et al. 2014 & M. Vallejo & Chiroptera & Mormoopidae & Pteronotus & parnelli & Brazil & 0,09 & 0,09 & NA-SA \\
\hline Castro et al. 2014 & M. Vallejo & Chiroptera & Natalidae & Natalus & macrourus & Brazil & 0,13 & 0,01 & NA-SA \\
\hline 71264 & J. Alroy & Chiroptera & Phyllostomidae & Artibeus & jamaicensis & Mexico & 0,13 & 0,01 & SA-NA \\
\hline 20350 & J. Alroy & Chiroptera & Phyllostomidae & Desmodus & archaeodaptes & United States & 1,80 & 0,30 & SA-NA \\
\hline 20351 & J. Alroy & Chiroptera & Phyllostomidae & Desmodus & archaeodaptes & United States & 1,80 & 0,30 & SA-NA \\
\hline 20384 & J. Alroy & Chiroptera & Phyllostomidae & Desmodus & archaeodaptes & United States & 4,90 & 1,80 & SA-NA \\
\hline 145845 & C. Jaramillo & Chiroptera & Phyllostomidae & Desmodus & archaeodaptes & United States & 1,80 & 0,30 & SA-NA \\
\hline 20491 & J. Alroy & Chiroptera & Phyllostomidae & Desmodus & stocki & United States & 0,30 & 0,01 & SA-NA \\
\hline 20573 & J. Alroy & Chiroptera & Phyllostomidae & Desmodus & stocki & United States & 0,13 & 0,01 & SA-NA \\
\hline 20595 & J. Alroy & Chiroptera & Phyllostomidae & Desmodus & stocki & United States & 0,13 & 0,01 & SA-NA \\
\hline 74497 & J. Alroy & Chiroptera & Phyllostomidae & Desmodus & stocki & United States & 0,13 & 0,01 & SA-NA \\
\hline 76060 & J. Alroy & Chiroptera & Phyllostomidae & Desmodus & stocki & United States & 0,13 & 0,01 & SA-NA \\
\hline 93332 & J. Alroy & Chiroptera & Phyllostomidae & Desmodus & stocki & United States & 0,03 & 0,03 & SA-NA \\
\hline 93332 & J. Alroy & Chiroptera & Phyllostomidae & Desmodus & sp. & United States & 0,03 & 0,03 & SA-NA \\
\hline 71313 & J. Alroy & Chiroptera & Vespertilionidae & Eptesicus & brasiliensis & Brazil & 0,02 & 0,02 & NA-SA \\
\hline 63515 & C. Bell & Chiroptera & Vespertilionidae & Eptesicus & fuscus & Venezuela & 0,13 & 0,01 & NA-SA \\
\hline 71346 & J. Alroy & Chiroptera & Vespertilionidae & Eptesicus & sp. & Peru & 0,13 & 0,01 & NA-SA \\
\hline 63515 & C. Bell & Chiroptera & Vespertilionidae & Rhogeessa & sp. & Venezuela & 0,13 & 0,01 & NA-SA \\
\hline 71305 & J. Alroy & Didelphimorph & Didelphidae & Marmosa & canescens & Mexico & 2,59 & 0,01 & SA-NA \\
\hline 71264 & J. Alroy & Didelphimorph & i Didelphidae & Marmosa & mexicana & Mexico & 0,13 & 0,01 & SA-NA \\
\hline 71334 & J. Alroy & Lagomorpha & Leporidae & Kerodon & rupestris & Brazil & 0,13 & 0,01 & NA-SA \\
\hline 61308 & D. Croft & Lagomorpha & Leporidae & Lepus & europaeus & Argentina & 0,01 & 0,00 & NA-SA \\
\hline 71326 & J. Alroy & Lagomorpha & Leporidae & Sylvilagus & brasiliensis & Peru & 0,01 & 0,00 & NA-SA \\
\hline 71327 & J. Alroy & Lagomorpha & Leporidae & Sylvilagus & brasiliensis & Peru & 0,01 & 0,01 & NA-SA \\
\hline 71328 & J. Alroy & Lagomorpha & Leporidae & Sylvilagus & brasiliensis & Peru & 0,01 & 0,01 & NA-SA \\
\hline 71329 & J. Alroy & Lagomorpha & Leporidae & Sylvilagus & brasiliensis & Peru & 0,01 & 0,01 & NA-SA \\
\hline 71285 & J. Alroy & Lagomorpha & Leporidae & Sylvilagus & floridanus & Colombia & 0,78 & 0,01 & NA-SA \\
\hline 147815 & C. Jaramillo & Lagomorpha & Leporidae & Sylvilagus & sp. & Brazil & 2,59 & 0,00 & NA-SA \\
\hline 70118 & J. Alroy & Notoungulata & Toxodontidae & Mixotoxodon & larensis & El Salvador & 2,59 & 0,13 & SA-NA \\
\hline 71267 & J. Alroy & Notoungulata & Toxodontidae & Mixotoxodon & larensis & Nicaragua & 0,13 & 0,01 & SA-NA \\
\hline 86959 & M. Uhen & Notoungulata & Toxodontidae & Mixotoxodon & larensis & Nicaragua & 2,59 & 0,01 & SA-NA \\
\hline Lucas 2014 & M. Vallejo & Notoungulata & Toxodontidae & Mixotoxodon & larensis & Panama & 0,47 & 0,45 & SA-NA \\
\hline 20152 & J. Alroy & Notoungulata & Toxodontidae & Mixotoxodon & sp. & El Salvador & 1,80 & 0,30 & SA-NA \\
\hline 20395 & J. Alroy & Notoungulata & Toxodontidae & Toxodon & sp. & Panama & 1,80 & 0,30 & SA-NA \\
\hline 71294 & J. Alroy & Notoungulata & Toxodontidae & Toxodon & sp. & Guatemala & 0,13 & 0,01 & SA-NA \\
\hline 142016 & C. Jaramillo & Perissodactyla & Equidae & Equus & andium & Bolivia & 2,59 & 0,78 & NA-SA \\
\hline Pino et al. 2013 & M. Vallejo & Perissodactyla & Equidae & Equus & andium & Chile & 0,01 & 0,01 & NA-SA \\
\hline 133633 & C. Jaramillo & Perissodactyla & Equidae & Equus & andium & Ecuador & 2,59 & 0,01 & NA-SA \\
\hline 133634 & C. Jaramillo & Perissodactyla & Equidae & Equus & andium & Ecuador & 2,59 & 0,01 & NA-SA \\
\hline 133635 & C. Jaramillo & Perissodactyla & Equidae & Equus & andium & Ecuador & 2,59 & 0,01 & NA-SA \\
\hline 133638 & C. Jaramillo & Perissodactyla & Equidae & Equus & andium & Ecuador & 2,59 & 0,01 & NA-SA \\
\hline 133639 & C. Jaramillo & Perissodactyla & Equidae & Equus & andium & Ecuador & 2,59 & 0,01 & NA-SA \\
\hline 133644 & C. Jaramillo & Perissodactyla & Equidae & Equus & andium & Ecuador & 2,59 & 0,01 & NA-SA \\
\hline 133653 & C. Jaramillo & Perissodactyla & Equidae & Equus & andium & Ecuador & 2,59 & 0,01 & NA-SA \\
\hline 133656 & C. Jaramillo & Perissodactyla & Equidae & Equus & andium & Ecuador & 2,59 & 0,01 & NA-SA \\
\hline 133657 & C. Jaramillo & Perissodactyla & Equidae & Equus & andium & Ecuador & 2,59 & 0,01 & NA-SA \\
\hline 133658 & C. Jaramillo & Perissodactyla & Equidae & Equus & andium & Ecuador & 2,59 & 0,01 & NA-SA \\
\hline 71279 & J. Alroy & Perissodactyla & Equidae & Equus & caballus & Argentina & 0,78 & 0,01 & NA-SA \\
\hline 71303 & J. Alroy & Perissodactyla & Equidae & Equus & caballus & Argentina & 0,78 & 0,01 & NA-SA \\
\hline 148309 & C. Jaramillo & Perissodactyla & Equidae & Equus & caballus & Brazil & 0,13 & 0,00 & NA-SA \\
\hline 148417 & C. Jaramillo & Perissodactyla & Equidae & Equus & caballus & Brazil & 0,13 & 0,01 & NA-SA \\
\hline Kerber et al. 2014 & M. Vallejo & Perissodactyla & Equidae & Equus & caballus & Brazil & 0,04 & 0,01 & NA-SA \\
\hline 71331 & J. Alroy & Perissodactyla & Equidae & Equus & caballus & Brazil & 0,13 & 0,01 & NA-SA \\
\hline 71332 & J. Alroy & Perissodactyla & Equidae & Equus & caballus & Brazil & 0,13 & 0,01 & NA-SA \\
\hline
\end{tabular}




\begin{tabular}{|c|c|c|c|c|c|c|c|c|c|}
\hline 71334 & J. Alroy & Perissodactyla & Equidae & Equus & caballus & Brazil & 0,13 & 0,01 & NA-SA \\
\hline 78577 & J. Alroy & Perissodactyla & Equidae & Equus & caballus & Argentina & 0,78 & 0,01 & NA-SA \\
\hline 123831 & M. Uhen & Perissodactyla & Equidae & Equus & caballus & Argentina & 2,59 & 0,01 & NA-SA \\
\hline 78580 & J. Alroy & Perissodactyla & Equidae & Equus & caballus & Argentina & 0,78 & 0,01 & NA-SA \\
\hline 63337 & J. Alroy & Perissodactyla & Equidae & Equus & caballus & Argentina & 0,78 & 0,01 & NA-SA \\
\hline 142016 & C. Jaramillo & Perissodactyla & Equidae & Equus & caballus & Bolivia & 2,59 & 0,78 & NA-SA \\
\hline 140934 & C. Jaramillo & Perissodactyla & Equidae & Equus & curvidens & Argentina & 0,78 & 0,01 & NA-SA \\
\hline 73842 & M. Uhen & Perissodactyla & Equidae & Equus & curvidens & Chile & 5,33 & 2,59 & NA-SA \\
\hline 70704 & D. Croft & Perissodactyla & Equidae & Equus & curvidens & Bolivia & 0,78 & 0,13 & NA-SA \\
\hline 142327 & C. Jaramillo & Perissodactyla & Equidae & Equus & insulatus & Bolivia & 0,13 & 0,01 & NA-SA \\
\hline 70673 & D. Croft & Perissodactyla & Equidae & Equus & insulatus & Bolivia & 2,59 & 0,78 & NA-SA \\
\hline 133634 & C. Jaramillo & Perissodactyla & Equidae & Equus & martinei & Ecuador & 2,59 & 0,01 & NA-SA \\
\hline 133659 & C. Jaramillo & Perissodactyla & Equidae & Equus & martinei & Ecuador & 2,59 & 0,01 & NA-SA \\
\hline 133660 & C. Jaramillo & Perissodactyla & Equidae & Equus & martinei & Ecuador & 2,59 & 0,01 & NA-SA \\
\hline 133661 & C. Jaramillo & Perissodactyla & Equidae & Equus & martinei & Ecuador & 2,59 & 0,01 & NA-SA \\
\hline 144922 & C. Jaramillo & Perissodactyla & Equidae & Equus & santaeelenae & Peru & 2,59 & 0,01 & NA-SA \\
\hline 145505 & C. Jaramillo & Perissodactyla & Equidae & Equus & santaeelenae & Peru & 0,03 & 0,02 & NA-SA \\
\hline 145506 & C. Jaramillo & Perissodactyla & Equidae & Equus & santaeelenae & Peru & 2,59 & 0,01 & NA-SA \\
\hline 145507 & C. Jaramillo & Perissodactyla & Equidae & Equus & santaeelenae & Peru & 2,59 & 0,01 & NA-SA \\
\hline 63515 & C. Bell & Perissodactyla & Equidae & Equus & santaeelenae & Venezuela & 0,13 & 0,01 & NA-SA \\
\hline Lindsey and Lopez 2014 & M. Vallejo & Perissodactyla & Equidae & Equus & santaeelenae & Ecuador & 0,78 & 0,01 & NA-SA \\
\hline 71283 & J. Alroy & Perissodactyla & Equidae & Equus & sp. & Venezuela & 0,01 & 0,01 & NA-SA \\
\hline 71284 & J. Alroy & Perissodactyla & Equidae & Equus & sp. & Venezuela & 0,13 & 0,01 & NA-SA \\
\hline 37999 & J. Alroy & Perissodactyla & Equidae & Equus & sp. & Argentina & 0,78 & 0,01 & NA-SA \\
\hline 71265 & J. Alroy & Perissodactyla & Equidae & Equus & sp. & Chile & 0,13 & 0,01 & NA-SA \\
\hline 71266 & J. Alroy & Perissodactyla & Equidae & Equus & sp. & Chile & 0,01 & 0,01 & NA-SA \\
\hline 71270 & J. Alroy & Perissodactyla & Equidae & Equus & sp. & Peru & 0,13 & 0,01 & NA-SA \\
\hline 71275 & J. Alroy & Perissodactyla & Equidae & Equus & sp. & Brazil & 2,59 & 0,01 & NA-SA \\
\hline 71276 & J. Alroy & Perissodactyla & Equidae & Equus & sp. & Brazil & 2,59 & 0,01 & NA-SA \\
\hline 71286 & J. Alroy & Perissodactyla & Equidae & Equus & sp. & Argentina & 0,78 & 0,01 & NA-SA \\
\hline 71287 & J. Alroy & Perissodactyla & Equidae & Equus & sp. & Argentina & 0,78 & 0,01 & NA-SA \\
\hline 71290 & J. Alroy & Perissodactyla & Equidae & Equus & sp. & Argentina & 0,78 & 0,01 & NA-SA \\
\hline 71296 & J. Alroy & Perissodactyla & Equidae & Equus & sp. & Peru & 0,13 & 0,01 & NA-SA \\
\hline 71298 & J. Alroy & Perissodactyla & Equidae & Equus & sp. & Brazil & 2,59 & 0,01 & NA-SA \\
\hline 71301 & J. Alroy & Perissodactyla & Equidae & Equus & sp. & Brazil & 2,59 & 0,01 & NA-SA \\
\hline 71302 & J. Alroy & Perissodactyla & Equidae & Equus & sp. & Brazil & 2,59 & 0,01 & NA-SA \\
\hline 71314 & J. Alroy & Perissodactyla & Equidae & Equus & sp. & Argentina & 0,78 & 0,01 & NA-SA \\
\hline 71322 & J. Alroy & Perissodactyla & Equidae & Equus & sp. & Bolivia & 1,20 & 0,78 & NA-SA \\
\hline 71346 & J. Alroy & Perissodactyla & Equidae & Equus & sp. & Peru & 0,13 & 0,01 & NA-SA \\
\hline 92814 & J. Alroy & Perissodactyla & Equidae & Equus & sp. & Brazil & 2,59 & 0,00 & NA-SA \\
\hline 140934 & C. Jaramillo & Perissodactyla & Equidae & Equus & sp. & Argentina & 0,78 & 0,01 & NA-SA \\
\hline 142016 & C. Jaramillo & Perissodactyla & Equidae & Equus & sp. & Bolivia & 2,59 & 0,78 & NA-SA \\
\hline 142158 & C. Jaramillo & Perissodactyla & Equidae & Equus & sp. & Bolivia & 0,13 & 0,01 & NA-SA \\
\hline 142160 & C. Jaramillo & Perissodactyla & Equidae & Equus & sp. & Bolivia & 0,13 & 0,01 & NA-SA \\
\hline 144517 & P. Mannion & Perissodactyla & Equidae & Equus & sp. & Argentina & 2,59 & 0,01 & NA-SA \\
\hline 144711 & C. Jaramillo & Perissodactyla & Equidae & Equus & sp. & Chile & 2,59 & 0,01 & NA-SA \\
\hline 144976 & C. Jaramillo & Perissodactyla & Equidae & Equus & sp. & Peru & 0,13 & 0,01 & NA-SA \\
\hline 145500 & C. Jaramillo & Perissodactyla & Equidae & Equus & sp. & Peru & 0,01 & 0,01 & NA-SA \\
\hline 145506 & C. Jaramillo & Perissodactyla & Equidae & Equus & sp. & Peru & 2,59 & 0,01 & NA-SA \\
\hline 146602 & C. Jaramillo & Perissodactyla & Equidae & Equus & sp. & Peru & 0,02 & 0,01 & NA-SA \\
\hline Luna and Cruz 2014 & M. Vallejo & Perissodactyla & Equidae & Equus & sp. & Argentina & 0,78 & 0,00 & NA-SA \\
\hline 70673 & D. Croft & Perissodactyla & Equidae & Hippidion & bonaerensis & Bolivia & 2,59 & 0,78 & NA-SA \\
\hline 71332 & J. Alroy & Perissodactyla & Equidae & Hippidion & bonaerensis & Brazil & 0,13 & 0,01 & NA-SA \\
\hline 71334 & J. Alroy & Perissodactyla & Equidae & Hippidion & bonaerensis & Brazil & 0,13 & 0,01 & NA-SA \\
\hline 71335 & J. Alroy & Perissodactyla & Equidae & Hippidion & bonaerensis & Brazil & 0,13 & 0,01 & NA-SA \\
\hline 71304 & J. Alroy & Perissodactyla & Equidae & Hippidion & chapalmalensis & Argentina & 2,59 & 0,01 & NA-SA \\
\hline 141896 & C. Jaramillo & Perissodactyla & Equidae & Hippidion & devillei & Argentina & 3,00 & 1,20 & NA-SA \\
\hline 141898 & C. Jaramillo & Perissodactyla & Equidae & Hippidion & devillei & Argentina & 1,20 & 0,78 & NA-SA \\
\hline 73842 & M. Uhen & Perissodactyla & Equidae & Hippidion & nannum & Chile & 5,33 & 2,59 & NA-SA \\
\hline 63335 & D. Croft & Perissodactyla & Equidae & Hippidion & principale & Argentina & 0,78 & 0,13 & NA-SA \\
\hline 70673 & D. Croft & Perissodactyla & Equidae & Hippidion & principale & Bolivia & 2,59 & 0,78 & NA-SA \\
\hline 73844 & J. Alroy & Perissodactyla & Equidae & Hippidion & saldiasi & Argentina & 0,13 & 0,01 & NA-SA \\
\hline 70704 & D. Croft & Perissodactyla & Equidae & Hippidion & sp. & Bolivia & 0,78 & 0,13 & NA-SA \\
\hline 71271 & J. Alroy & Perissodactyla & Equidae & Hippidion & sp. & Argentina & 0,13 & 0,01 & NA-SA \\
\hline 28513 & J. Alroy & Perissodactyla & Equidae & Hippidion & sp. & Brazil & 2,59 & 0,01 & NA-SA \\
\hline 71274 & J. Alroy & Perissodactyla & Equidae & Hippidion & sp. & Uruguay & 0,78 & 0,01 & NA-SA \\
\hline 71280 & J. Alroy & Perissodactyla & Equidae & Hippidion & sp. & Chile & 0,13 & 0,01 & NA-SA \\
\hline 71284 & J. Alroy & Perissodactyla & Equidae & Hippidion & sp. & Venezuela & 0,13 & 0,01 & NA-SA \\
\hline 71295 & J. Alroy & Perissodactyla & Equidae & Hippidion & sp. & Brazil & 0,03 & 0,20 & NA-SA \\
\hline 71301 & J. Alroy & Perissodactyla & Equidae & Hippidion & sp. & Brazil & 2,59 & 0,01 & NA-SA \\
\hline 71302 & J. Alroy & Perissodactyla & Equidae & Hippidion & sp. & Brazil & 2,59 & 0,01 & NA-SA \\
\hline 71316 & J. Alroy & Perissodactyla & Equidae & Hippidion & sp. & Argentina & 0,13 & 0,01 & NA-SA \\
\hline 71322 & J. Alroy & Perissodactyla & Equidae & Hippidion & sp. & Bolivia & 1,20 & 0,78 & NA-SA \\
\hline 71336 & J. Alroy & Perissodactyla & Equidae & Hippidion & sp. & Argentina & 0,13 & 0,01 & NA-SA \\
\hline 71337 & J. Alroy & Perissodactyla & Equidae & Hippidion & sp. & Argentina & 0,01 & 0,01 & NA-SA \\
\hline 71338 & J. Alroy & Perissodactyla & Equidae & Hippidion & sp. & Argentina & 0,13 & 0,01 & NA-SA \\
\hline 71339 & J. Alroy & Perissodactyla & Equidae & Hippidion & sp. & Argentina & 0,13 & 0,01 & NA-SA \\
\hline 71340 & J. Alroy & Perissodactyla & Equidae & Hippidion & sp. & Chile & 0,13 & 0,00 & NA-SA \\
\hline 71341 & J. Alroy & Perissodactyla & Equidae & Hippidion & sp. & Chile & 0,01 & 0,01 & NA-SA \\
\hline
\end{tabular}




\begin{tabular}{|c|c|c|c|c|c|c|c|c|c|}
\hline 71342 & J. Alroy & Perissodactyla & Equidae & Hippidion & sp. & Chile & 0,13 & 0,01 & NA-SA \\
\hline 71343 & J. Alroy & Perissodactyla & Equidae & Hippidion & sp. & Argentina & 0,01 & 0,01 & NA-SA \\
\hline 71344 & J. Alroy & Perissodactyla & Equidae & Hippidion & sp. & Chile & 0,13 & 0,01 & NA-SA \\
\hline 120884 & M. Uhen & Perissodactyla & Equidae & Hippidion & sp. & Uruguay & 0,78 & 0,01 & NA-SA \\
\hline 140809 & C. Jaramillo & Perissodactyla & Equidae & Hippidion & sp. & Argentina & 0,13 & 0,01 & NA-SA \\
\hline 140909 & C. Jaramillo & Perissodactyla & Equidae & Hippidion & sp. & Argentina & 0,13 & 0,01 & NA-SA \\
\hline 141367 & C. Jaramillo & Perissodactyla & Equidae & Hippidion & sp. & Chile & 0,13 & 0,01 & NA-SA \\
\hline 147609 & C. Jaramillo & Perissodactyla & Equidae & Hippidion & sp. & Brazil & 2,59 & 0,00 & NA-SA \\
\hline 148309 & C. Jaramillo & Perissodactyla & Equidae & Hippidion & sp. & Brazil & 0,13 & 0,00 & NA-SA \\
\hline 148417 & C. Jaramillo & Perissodactyla & Equidae & Hippidion & sp. & Brazil & 0,13 & 0,01 & NA-SA \\
\hline Kerber et al. 2014 & M. Vallejo & Perissodactyla & Equidae & Hippidion & sp. & Brazil & 0,04 & 0,01 & NA-SA \\
\hline 63761 & D. Croft & Perissodactyla & Equidae & Onohippidium & bolivianum & Bolivia & 2,59 & 0,01 & NA-SA \\
\hline 70673 & D. Croft & Perissodactyla & Equidae & Onohippidium & devillei & Bolivia & 2,59 & 0,78 & NA-SA \\
\hline 142016 & C. Jaramillo & Perissodactyla & Equidae & Onohippidium & devillei & Bolivia & 2,59 & 0,78 & NA-SA \\
\hline 145508 & C. Jaramillo & Perissodactyla & Equidae & Onohippidion & peruanum & Peru & 2,59 & 0,01 & NA-SA \\
\hline 53926 & M. Uhen & Perissodactyla & Equidae & Onohippidion & saldiasi & Argentina & 3,60 & 2,59 & NA-SA \\
\hline 71304 & J. Alroy & Perissodactyla & Equidae & Onohippidium & sp. & Argentina & 2,59 & 0,01 & NA-SA \\
\hline 53926 & M. Uhen & Perissodactyla & Equidae & Onohippidion & sp. & Argentina & 3,60 & 2,59 & NA-SA \\
\hline 53926 & M. Uhen & Perissodactyla & Tapiridae & Tapirus & greslebini & Argentina & 3,60 & 2,59 & NA-SA \\
\hline 93265 & J. Alroy & Perissodactyla & Tapiridae & Tapirus & mesopotamicus & Argentina & 0,13 & 0,01 & NA-SA \\
\hline 146534 & C. Jaramillo & Perissodactyla & Tapiridae & Tapirus & olivaresi & Uruguay & 0,13 & 0,01 & NA-SA \\
\hline 70673 & D. Croft & Perissodactyla & Tapiridae & Tapirus & tarijensis & Bolivia & 2,59 & 0,78 & NA-SA \\
\hline 71291 & J. Alroy & Perissodactyla & Tapiridae & Tapirus & terrestris & Colombia & 0,78 & 0,01 & NA-SA \\
\hline 71297 & J. Alroy & Perissodactyla & Tapiridae & Tapirus & terrestris & Brazil & 2,59 & 0,01 & NA-SA \\
\hline 136498 & C. Jaramillo & Perissodactyla & Tapiridae & Tapirus & terrestris & Brazil & 0,13 & 0,00 & NA-SA \\
\hline 142016 & C. Jaramillo & Perissodactyla & Tapiridae & Tapirus & terrestris & Bolivia & 2,59 & 0,78 & NA-SA \\
\hline 147190 & C. Jaramillo & Perissodactyla & Tapiridae & Tapirus & terrestris & Venezuela & 2,59 & 0,01 & NA-SA \\
\hline 147609 & C. Jaramillo & Perissodactyla & Tapiridae & Tapirus & terrestris & Brazil & 2,59 & 0,00 & NA-SA \\
\hline DeOliveira et al. 2014 & M. Vallejo & Perissodactyla & Tapiridae & Tapirus & terrestris & Brazil & 0,01 & 0,01 & NA-SA \\
\hline 71274 & J. Alroy & Perissodactyla & Tapiridae & Tapirus & sp. & Uruguay & 0,78 & 0,01 & NA-SA \\
\hline 71346 & J. Alroy & Perissodactyla & Tapiridae & Tapirus & sp. & Peru & 0,13 & 0,01 & NA-SA \\
\hline 92814 & J. Alroy & Perissodactyla & Tapiridae & Tapirus & sp. & Brazil & 2,59 & 0,00 & NA-SA \\
\hline 92815 & J. Alroy & Perissodactyla & Tapiridae & Tapirus & sp. & Brazil & 2,59 & 0,00 & NA-SA \\
\hline 145500 & C. Jaramillo & Perissodactyla & Tapiridae & Tapirus & sp. & Peru & 0,01 & 0,01 & NA-SA \\
\hline 147081 & C. Jaramillo & Perissodactyla & Tapiridae & Tapirus & sp. & Venezuela & 2,59 & 0,13 & NA-SA \\
\hline Kerber et al. 2014 & M. Vallejo & Perissodactyla & Tapiridae & Tapirus & sp. & Brazil & 0,04 & 0,01 & NA-SA \\
\hline 22734 & A. Behrensmeyer & Proboscidea & Gomphotheriidae & Cuvieronius & andium & Ecuador & 2,59 & 0,01 & NA-SA \\
\hline 133636 & C. Jaramillo & Proboscidea & Gomphotheriidae & Cuvieronius & hyodon & Ecuador & 2,59 & 0,01 & NA-SA \\
\hline 133644 & C. Jaramillo & Proboscidea & Gomphotheriidae & Cuvieronius & hyodon & Ecuador & 2,59 & 0,01 & NA-SA \\
\hline 133646 & C. Jaramillo & Proboscidea & Gomphotheriidae & Cuvieronius & hyodon & Ecuador & 2,59 & 0,01 & NA-SA \\
\hline 142016 & C. Jaramillo & Proboscidea & Gomphotheriidae & Cuvieronius & hyodon & Bolivia & 2,59 & 0,78 & NA-SA \\
\hline 142158 & C. Jaramillo & Proboscidea & Gomphotheriidae & Cuvieronius & hyodon & Bolivia & 0,13 & 0,01 & NA-SA \\
\hline 142160 & C. Jaramillo & Proboscidea & Gomphotheriidae & Cuvieronius & hyodon & Bolivia & 0,13 & 0,01 & NA-SA \\
\hline 63761 & D. Croft & Proboscidea & Gomphotheriidae & Cuvieronius & tropicus & Bolivia & 2,59 & 0,01 & NA-SA \\
\hline 73842 & M. Uhen & Proboscidea & Gomphotheriidae & Cuvieronius & tropicus & Chile & 5,33 & 2,59 & NA-SA \\
\hline 70673 & D. Croft & Proboscidea & Gomphotheriidae & Cuvieronius & tropicus & Bolivia & 2,59 & 0,78 & NA-SA \\
\hline 71265 & J. Alroy & Proboscidea & Gomphotheriidae & Cuvieronius & sp. & Chile & 0,13 & 0,01 & NA-SA \\
\hline 71266 & J. Alroy & Proboscidea & Gomphotheriidae & Cuvieronius & sp. & Chile & 0,01 & 0,01 & NA-SA \\
\hline 71590 & D. Croft & Proboscidea & Gomphotheriidae & Cuvieronius & sp. & Bolivia & 2,59 & 0,01 & NA-SA \\
\hline 146402 & C. Jaramillo & Proboscidea & Gomphotheriidae & Cuvieronius & sp. & Venezuela & 0,13 & 0,01 & NA-SA \\
\hline 67633 & M. Uhen & Proboscidea & Gomphotheriidae & Haplomastodon & chimborazi & Ecuador & 0,78 & 0,01 & NA-SA \\
\hline 71275 & J. Alroy & Proboscidea & Gomphotheriidae & Haplomastodon & chimborazi & Brazil & 2,59 & 0,01 & NA-SA \\
\hline 71291 & J. Alroy & Proboscidea & Gomphotheriidae & Haplomastodon & chimborazi & Colombia & 0,78 & 0,01 & NA-SA \\
\hline 71293 & J. Alroy & Proboscidea & Gomphotheriidae & Haplomastodon & chimborazi & Brazil & 2,59 & 0,01 & NA-SA \\
\hline 71335 & J. Alroy & Proboscidea & Gomphotheriidae & Haplomastodon & chimborazi & Brazil & 0,13 & 0,01 & NA-SA \\
\hline 71276 & J. Alroy & Proboscidea & Gomphotheriidae & Haplomastodon & chimborazi & Brazil & 2,59 & 0,01 & NA-SA \\
\hline 71350 & J. Alroy & Proboscidea & Gomphotheriidae & Haplomastodon & chimborazi & Venezuela & 0,13 & 0,01 & NA-SA \\
\hline 133632 & C. Jaramillo & Proboscidea & Gomphotheriidae & Haplomastodon & chimborazi & Ecuador & 2,59 & 0,01 & NA-SA \\
\hline 133634 & C. Jaramillo & Proboscidea & Gomphotheriidae & Haplomastodon & chimborazi & Ecuador & 2,59 & 0,01 & NA-SA \\
\hline 133635 & C. Jaramillo & Proboscidea & Gomphotheriidae & Haplomastodon & chimborazi & Ecuador & 2,59 & 0,01 & NA-SA \\
\hline 133639 & C. Jaramillo & Proboscidea & Gomphotheriidae & Haplomastodon & chimborazi & Ecuador & 2,59 & 0,01 & NA-SA \\
\hline 133644 & C. Jaramillo & Proboscidea & Gomphotheriidae & Haplomastodon & chimborazi & Ecuador & 2,59 & 0,01 & NA-SA \\
\hline 133647 & C. Jaramillo & Proboscidea & Gomphotheriidae & Haplomastodon & chimborazi & Ecuador & 2,59 & 0,01 & NA-SA \\
\hline 133653 & C. Jaramillo & Proboscidea & Gomphotheriidae & Haplomastodon & chimborazi & Ecuador & 2,59 & 0,01 & NA-SA \\
\hline 133654 & C. Jaramillo & Proboscidea & Gomphotheriidae & Haplomastodon & chimborazi & Ecuador & 2,59 & 0,01 & NA-SA \\
\hline 133655 & C. Jaramillo & Proboscidea & Gomphotheriidae & Haplomastodon & chimborazi & Ecuador & 2,59 & 0,01 & NA-SA \\
\hline 133630 & C. Jaramillo & Proboscidea & Gomphotheriidae & Haplomastodon & guayasensis & Ecuador & 0,78 & 0,01 & NA-SA \\
\hline 145518 & C. Jaramillo & Proboscidea & Gomphotheriidae & Haplomastodon & waringi & Colombia & 2,59 & 0,01 & NA-SA \\
\hline 146529 & C. Jaramillo & Proboscidea & Gomphotheriidae & Haplomastodon & waringi & Colombia & 2,59 & 0,01 & NA-SA \\
\hline 151541 & C. Jaramillo & Proboscidea & Gomphotheriidae & Haplomastodon & waringi & Brazil & 2,59 & 0,01 & NA-SA \\
\hline 71282 & J. Alroy & Proboscidea & Gomphotheriidae & Haplomastodon & sp. & Venezuela & 0,13 & 0,01 & NA-SA \\
\hline 71283 & J. Alroy & Proboscidea & Gomphotheriidae & Haplomastodon & sp. & Venezuela & 0,01 & 0,01 & NA-SA \\
\hline 71290 & J. Alroy & Proboscidea & Gomphotheriidae & Haplomastodon & sp. & Argentina & 0,78 & 0,01 & NA-SA \\
\hline 71299 & J. Alroy & Proboscidea & Gomphotheriidae & Haplomastodon & sp. & Brazil & 2,59 & 0,01 & NA-SA \\
\hline 71332 & J. Alroy & Proboscidea & Gomphotheriidae & Haplomastodon & sp. & Brazil & 0,13 & 0,01 & NA-SA \\
\hline 70673 & D. Croft & Proboscidea & Gomphotheriidae & Haplomastodon & sp. & Bolivia & 2,59 & 0,78 & NA-SA \\
\hline 71346 & J. Alroy & Proboscidea & Gomphotheriidae & Haplomastodon & sp. & Peru & 0,13 & 0,01 & NA-SA \\
\hline 144976 & C. Jaramillo & Proboscidea & Gomphotheriidae & Haplomastodon & sp. & Peru & 0,13 & 0,01 & NA-SA \\
\hline 70673 & D. Croft & Proboscidea & Gomphotheriidae & Notiomastodon & sp. & Bolivia & 2,59 & 0,78 & NA-SA \\
\hline
\end{tabular}




\begin{tabular}{|c|c|c|c|c|c|c|c|c|c|}
\hline 78578 & J. Alroy & Proboscidea & Gomphotheriidae & Stegomastodon & platensis & Argentina & 0,78 & 0,01 & NA-SA \\
\hline Lindsey and Lopez 2014 & M. Vallejo & Proboscidea & Gomphotheriidae & Stegomastodon & platensis & Ecuador & 0,78 & 0,01 & NA-SA \\
\hline 140923 & C. Jaramillo & Proboscidea & Gomphotheriidae & Stegomastodon & platensis & Argentina & 2,59 & 0,01 & NA-SA \\
\hline 78579 & J. Alroy & Proboscidea & Gomphotheriidae & Stegomastodon & platensis & Argentina & 0,78 & 0,01 & NA-SA \\
\hline 71303 & J. Alroy & Proboscidea & Gomphotheriidae & Stegomastodon & superbus & Argentina & 0,78 & 0,01 & NA-SA \\
\hline 93235 & M. Uhen & Proboscidea & Gomphotheriidae & Stegomastodon & waringi & Brazil & 0,13 & 0,00 & NA-SA \\
\hline 120884 & M. Uhen & Proboscidea & Gomphotheriidae & Stegomastodon & waringi & Uruguay & 0,78 & 0,01 & NA-SA \\
\hline 136420 & C. Jaramillo & Proboscidea & Gomphotheriidae & Stegomastodon & waringi & Brazil & 0,13 & 0,00 & NA-SA \\
\hline 136498 & C. Jaramillo & Proboscidea & Gomphotheriidae & Stegomastodon & waringi & Brazil & 0,13 & 0,00 & NA-SA \\
\hline 137121 & C. Jaramillo & Proboscidea & Gomphotheriidae & Stegomastodon & waringi & Brazil & 0,13 & 0,01 & NA-SA \\
\hline 139660 & C. Jaramillo & Proboscidea & Gomphotheriidae & Stegomastodon & waringi & Uruguay & 0,13 & 0,01 & NA-SA \\
\hline 140354 & C. Jaramillo & Proboscidea & Gomphotheriidae & Stegomastodon & waringi & Brazil & 0,13 & 0,01 & NA-SA \\
\hline 140355 & C. Jaramillo & Proboscidea & Gomphotheriidae & Stegomastodon & waringi & Brazil & 0,13 & 0,01 & NA-SA \\
\hline 140356 & C. Jaramillo & Proboscidea & Gomphotheriidae & Stegomastodon & waringi & Brazil & 0,13 & 0,01 & NA-SA \\
\hline 140357 & C. Jaramillo & Proboscidea & Gomphotheriidae & Stegomastodon & waringi & Brazil & 0,13 & 0,01 & NA-SA \\
\hline 145502 & C. Jaramillo & Proboscidea & Gomphotheriidae & Stegomastodon & waringi & Peru & 0,78 & 0,01 & NA-SA \\
\hline 140358 & C. Jaramillo & Proboscidea & Gomphotheriidae & Stegomastodon & waringi & Brazil & 0,13 & 0,01 & NA-SA \\
\hline 145506 & C. Jaramillo & Proboscidea & Gomphotheriidae & Stegomastodon & waringi & Peru & 2,59 & 0,01 & NA-SA \\
\hline 145507 & C. Jaramillo & Proboscidea & Gomphotheriidae & Stegomastodon & waringi & Peru & 2,59 & 0,01 & NA-SA \\
\hline 146528 & C. Jaramillo & Proboscidea & Gomphotheriidae & Stegomastodon & waringi & Colombia & 2,59 & 0,01 & NA-SA \\
\hline 147605 & C. Jaramillo & Proboscidea & Gomphotheriidae & Stegomastodon & waringi & Brazil & 0,01 & 0,00 & NA-SA \\
\hline 147609 & C. Jaramillo & Proboscidea & Gomphotheriidae & Stegomastodon & waringi & Brazil & 2,59 & 0,00 & NA-SA \\
\hline 148310 & C. Jaramillo & Proboscidea & Gomphotheriidae & Stegomastodon & waringi & Brazil & 2,59 & 0,01 & NA-SA \\
\hline 22633 & A. Behrensmeyer & Proboscidea & Gomphotheriidae & Stegomastodon & waringi & Brazil & 2,59 & 0,01 & NA-SA \\
\hline 70704 & D. Croft & Proboscidea & Gomphotheriidae & Stegomastodon & sp. & Bolivia & 0,78 & 0,13 & NA-SA \\
\hline 78578 & J. Alroy & Proboscidea & Gomphotheriidae & Stegomastodon & sp. & Argentina & 0,78 & 0,01 & NA-SA \\
\hline 71322 & J. Alroy & Proboscidea & Gomphotheriidae & Stegomastodon & sp. & Bolivia & 1,20 & 0,78 & NA-SA \\
\hline 78576 & J. Alroy & Proboscidea & Gomphotheriidae & Stegomastodon & sp. & Argentina & 0,78 & 0,01 & NA-SA \\
\hline 78577 & J. Alroy & Proboscidea & Gomphotheriidae & Stegomastodon & sp. & Argentina & 0,78 & 0,01 & NA-SA \\
\hline 71282 & J. Alroy & Proboscidea & Gomphotheriidae & Stegomastodon & sp. & Venezuela & 0,13 & 0,01 & NA-SA \\
\hline 71283 & J. Alroy & Proboscidea & Gomphotheriidae & Stegomastodon & sp. & Venezuela & 0,01 & 0,01 & NA-SA \\
\hline 71288 & J. Alroy & Proboscidea & Gomphotheriidae & Stegomastodon & sp. & Argentina & 1,20 & 0,01 & NA-SA \\
\hline 71292 & J. Alroy & Proboscidea & Gomphotheriidae & Stegomastodon & sp. & Paraguay & 2,59 & 0,01 & NA-SA \\
\hline 144922 & C. Jaramillo & Proboscidea & Gomphotheriidae & Stegomastodon & sp. & Peru & 2,59 & 0,01 & NA-SA \\
\hline 145500 & C. Jaramillo & Proboscidea & Gomphotheriidae & Stegomastodon & sp. & Peru & 0,01 & 0,01 & NA-SA \\
\hline 145505 & C. Jaramillo & Proboscidea & Gomphotheriidae & Stegomastodon & sp. & Peru & 0,03 & 0,02 & NA-SA \\
\hline 145506 & C. Jaramillo & Proboscidea & Gomphotheriidae & Stegomastodon & sp. & Peru & 2,59 & 0,01 & NA-SA \\
\hline 145508 & C. Jaramillo & Proboscidea & Gomphotheriidae & Stegomastodon & sp. & Peru & 2,59 & 0,01 & NA-SA \\
\hline 146402 & C. Jaramillo & Proboscidea & Gomphotheriidae & Stegomastodon & sp. & Venezuela & 0,13 & 0,01 & NA-SA \\
\hline 151920 & C. Jaramillo & Proboscidea & Gomphotheriidae & Stegomastodon & sp. & Peru & 2,59 & 0,01 & NA-SA \\
\hline Kerber et al. 2014 & M. Vallejo & Proboscidea & Gomphotheriidae & & & Brazil & 0,04 & 0,01 & NA-SA \\
\hline Labarca et al. 2014 & M. Vallejo & Proboscidea & Gomphotheriidae & & & Chile & 0,01 & 0,01 & NA-SA \\
\hline 71584 & D. Croft & Proboscidea & Mammutidae & Mammut & andinum & Bolivia & 2,59 & 0,01 & NA-SA \\
\hline 71588 & D. Croft & Proboscidea & Mammutidae & Mammut & sp. & Bolivia & 2,59 & 0,01 & NA-SA \\
\hline 71589 & D. Croft & Proboscidea & Mammutidae & Mammut & sp. & Bolivia & 2,59 & 0,01 & NA-SA \\
\hline 71592 & D. Croft & Proboscidea & Mammutidae & Mammut & sp. & Bolivia & 2,59 & 0,01 & NA-SA \\
\hline 71593 & D. Croft & Proboscidea & Mammutidae & Mammut & sp. & Bolivia & 2,59 & 0,01 & NA-SA \\
\hline 20495 & J. Alroy & Rodentia & Caviidae & Hydrochoerus & sp. & Mexico & 0,30 & 0,01 & SA-NA \\
\hline 71312 & J. Alroy & Rodentia & Caviidae & Hydrochoerus & sp. & Guatemala & 0,13 & 0,01 & SA-NA \\
\hline 20384 & J. Alroy & Rodentia & Caviidae & Neochoerus & aesopi & United States & 4,90 & 1,80 & SA-NA \\
\hline 20289 & J. Alroy & Rodentia & Caviidae & Neochoerus & aesopi & United States & 1,80 & 0,30 & SA-NA \\
\hline 20491 & J. Alroy & Rodentia & Caviidae & Neochoerus & aesopi & United States & 0,30 & 0,01 & SA-NA \\
\hline 20520 & J. Alroy & Rodentia & Caviidae & Neochoerus & aesopi & United States & 1,80 & 0,30 & SA-NA \\
\hline 20638 & J. Alroy & Rodentia & Caviidae & Neochoerus & aesopi & United States & 0,13 & 0,01 & SA-NA \\
\hline 79760 & M. Uhen & Rodentia & Caviidae & Neochoerus & aesopi & United States & 0,30 & 0,01 & SA-NA \\
\hline 71278 & J. Alroy & Rodentia & Caviidae & Neochoerus & aesopi & Panama & 0,13 & 0,01 & SA-NA \\
\hline 19752 & J. Alroy & Rodentia & Caviidae & Neochoerus & aesopi & United States & 4,90 & 1,80 & SA-NA \\
\hline 80087 & M. Uhen & Rodentia & Caviidae & Neochoerus & aesopi & United States & 1,80 & 0,30 & SA-NA \\
\hline 80227 & M. Uhen & Rodentia & Caviidae & Neochoerus & aesopi & United States & 0,78 & 0,13 & SA-NA \\
\hline 80229 & M. Uhen & Rodentia & Caviidae & Neochoerus & aesopi & United States & 0,78 & 0,13 & SA-NA \\
\hline 80230 & M. Uhen & Rodentia & Caviidae & Neochoerus & aesopi & United States & 0,13 & 0,01 & SA-NA \\
\hline 80231 & M. Uhen & Rodentia & Caviidae & Neochoerus & aesopi & United States & 0,13 & 0,01 & SA-NA \\
\hline 86959 & M. Uhen & Rodentia & Caviidae & Neochoerus & aesopi & Nicaragua & 2,59 & 0,01 & SA-NA \\
\hline 79923 & M. Uhen & Rodentia & Caviidae & Neochoerus & aesopi & United States & 0,13 & 0,01 & SA-NA \\
\hline 20440 & J. Alroy & Rodentia & Caviidae & Neochoerus & pinckneyi & United States & 0,30 & 0,01 & SA-NA \\
\hline 20581 & J. Alroy & Rodentia & Caviidae & Neochoerus & pinckneyi & United States & 0,13 & 0,01 & SA-NA \\
\hline 20613 & J. Alroy & Rodentia & Caviidae & Neochoerus & pinckneyi & United States & 0,13 & 0,01 & SA-NA \\
\hline 65405 & M. Uhen & Rodentia & Caviidae & Neochoerus & pinckneyi & United States & 2,59 & 0,01 & SA-NA \\
\hline 80232 & M. Uhen & Rodentia & Caviidae & Neochoerus & pinckneyi & United States & 0,13 & 0,01 & SA-NA \\
\hline 79757 & M. Uhen & Rodentia & Caviidae & Neochoerus & pinckneyi & United States & 0,30 & 0,01 & SA-NA \\
\hline 79923 & M. Uhen & Rodentia & Caviidae & Neochoerus & pinckneyi & United States & 0,13 & 0,01 & SA-NA \\
\hline 80230 & M. Uhen & Rodentia & Caviidae & Neochoerus & pinckneyi & United States & 0,13 & 0,01 & SA-NA \\
\hline 19645 & J. Alroy & Rodentia & Caviidae & Neochoerus & sp. & United States & 4,90 & 1,80 & SA-NA \\
\hline 19750 & J. Alroy & Rodentia & Caviidae & Neochoerus & sp. & United States & 4,90 & 0,30 & SA-NA \\
\hline 20077 & J. Alroy & Rodentia & Caviidae & Neochoerus & sp. & Mexico & 4,90 & 1,80 & SA-NA \\
\hline 20145 & J. Alroy & Rodentia & Caviidae & Neochoerus & sp. & United States & 1,80 & 0,30 & SA-NA \\
\hline 20285 & J. Alroy & Rodentia & Caviidae & Neochoerus & sp. & Mexico & 1,80 & 0,30 & SA-NA \\
\hline 20322 & J. Alroy & Rodentia & Caviidae & Neochoerus & sp. & Mexico & 4,90 & 1,80 & SA-NA \\
\hline 70113 & J. Alroy & Rodentia & Caviidae & Neochoerus & sp. & Mexico & 4,90 & 1,80 & SA-NA \\
\hline
\end{tabular}




\begin{tabular}{|c|c|c|c|c|c|c|c|c|c|}
\hline 74470 & J. Alroy & Rodentia & Caviidae & Neochoerus & sp. & United States & 0,13 & 0,01 & SA-NA \\
\hline 19657 & J. Alroy & Rodentia & Caviidae & Phugatherium & dichroplax & United States & 4,90 & 1,80 & SA-NA \\
\hline 19658 & J. Alroy & Rodentia & Caviidae & Phugatherium & dichroplax & United States & 4,90 & 1,80 & SA-NA \\
\hline 19750 & J. Alroy & Rodentia & Caviidae & Phugatherium & dichroplax & United States & 4,90 & 0,30 & SA-NA \\
\hline 19753 & J. Alroy & Rodentia & Caviidae & Phugatherium & dichroplax & United States & 4,90 & 1,80 & SA-NA \\
\hline 19754 & J. Alroy & Rodentia & Caviidae & Phugatherium & dichroplax & United States & 4,90 & 1,80 & SA-NA \\
\hline 19756 & J. Alroy & Rodentia & Caviidae & Phugatherium & dichroplax & United States & 4,90 & 1,80 & SA-NA \\
\hline 19763 & J. Alroy & Rodentia & Caviidae & Phugatherium & dichroplax & United States & 4,90 & 1,80 & SA-NA \\
\hline 20077 & J. Alroy & Rodentia & Caviidae & Phugatherium & dichroplax & Mexico & 4,90 & 1,80 & SA-NA \\
\hline 20078 & J. Alroy & Rodentia & Caviidae & Phugatherium & dichroplax & Mexico & 4,90 & 0,30 & SA-NA \\
\hline 20539 & J. Alroy & Rodentia & Caviidae & Phugatherium & dichroplax & Mexico & 1,80 & 0,30 & SA-NA \\
\hline 98313 & M. Uhen & Rodentia & Caviidae & Phugatherium & dichroplax & United States & 4,90 & 0,30 & SA-NA \\
\hline 98320 & M. Uhen & Rodentia & Caviidae & Phugatherium & dichroplax & United States & 4,90 & 1,80 & SA-NA \\
\hline 141182 & C. Jaramillo & Rodentia & Cricetidae & Abrothrix & illuteus & Argentina & 0,03 & 0,02 & NA-SA \\
\hline 63335 & D. Croft & Rodentia & Cricetidae & Akodon & azarae & Argentina & 0,78 & 0,13 & NA-SA \\
\hline 145810 & C. Jaramillo & Rodentia & Cricetidae & Akodon & azarae & Argentina & 0,01 & 0,00 & NA-SA \\
\hline 71316 & J. Alroy & Rodentia & Cricetidae & Akodon & azarae & Argentina & 0,13 & 0,01 & NA-SA \\
\hline 71330 & J. Alroy & Rodentia & Cricetidae & Akodon & azarae & Argentina & 0,13 & 0,01 & NA-SA \\
\hline 63335 & D. Croft & Rodentia & Cricetidae & Akodon & iniscatus & Argentina & 0,78 & 0,13 & NA-SA \\
\hline 92813 & J. Alroy & Rodentia & Cricetidae & Akodon & sp. & Brazil & 2,59 & 0,00 & NA-SA \\
\hline 141182 & C. Jaramillo & Rodentia & Cricetidae & Akodon & sp. & Argentina & 0,03 & 0,02 & NA-SA \\
\hline 147815 & C. Jaramillo & Rodentia & Cricetidae & Akodon & sp. & Brazil & 2,59 & 0,00 & NA-SA \\
\hline 70673 & D. Croft & Rodentia & Cricetidae & Andinomys & edea & Bolivia & 2,59 & 0,78 & NA-SA \\
\hline 141182 & C. Jaramillo & Rodentia & Cricetidae & Andinomys & edax & Argentina & 0,03 & 0,02 & NA-SA \\
\hline 152062 & C. Jaramillo & Rodentia & Cricetidae & Auliscomys & formosus & Argentina & 6,80 & 4,00 & NA-SA \\
\hline 152081 & C. Jaramillo & Rodentia & Cricetidae & Auliscomys & formosus & Argentina & 11,61 & 3,60 & NA-SA \\
\hline 71303 & J. Alroy & Rodentia & Cricetidae & Calomys & callosus & Argentina & 0,78 & 0,01 & NA-SA \\
\hline 63515 & C. Bell & Rodentia & Cricetidae & Calomys & hummelinki & Venezuela & 0,13 & 0,01 & NA-SA \\
\hline 70673 & D. Croft & Rodentia & Cricetidae & Calomys & lancha & Bolivia & 2,59 & 0,78 & NA-SA \\
\hline 145810 & C. Jaramillo & Rodentia & Cricetidae & Calomys & musculinus & Argentina & 0,01 & 0,00 & NA-SA \\
\hline 70705 & D. Croft & Rodentia & Cricetidae & Calomys & sp. & Bolivia & 0,01 & 0,00 & NA-SA \\
\hline 71324 & J. Alroy & Rodentia & Cricetidae & Calomys & sp. & Bolivia & 0,01 & 0,01 & NA-SA \\
\hline 92813 & J. Alroy & Rodentia & Cricetidae & Calomys & sp. & Brazil & 2,59 & 0,00 & NA-SA \\
\hline 140807 & C. Jaramillo & Rodentia & Cricetidae & Calomys & sp. & Argentina & 0,78 & 0,01 & NA-SA \\
\hline 63338 & J. Alroy & Rodentia & Cricetidae & Calomys & sp. & Argentina & 0,01 & 0,00 & NA-SA \\
\hline 141182 & C. Jaramillo & Rodentia & Cricetidae & Calomys & sp. & Argentina & 0,03 & 0,02 & NA-SA \\
\hline 144922 & C. Jaramillo & Rodentia & Cricetidae & Calomys & sp. & Peru & 2,59 & 0,01 & NA-SA \\
\hline 140934 & C. Jaramillo & Rodentia & Cricetidae & Eligmodontia & typus & Argentina & 0,78 & 0,01 & NA-SA \\
\hline 70705 & D. Croft & Rodentia & Cricetidae & Graomys & sp. & Bolivia & 0,01 & 0,00 & NA-SA \\
\hline 63338 & J. Alroy & Rodentia & Cricetidae & Holochilus & brasiliensis & Argentina & 0,01 & 0,00 & NA-SA \\
\hline 70705 & D. Croft & Rodentia & Cricetidae & Holochilus & brasilienses & Bolivia & 0,01 & 0,00 & NA-SA \\
\hline 92813 & J. Alroy & Rodentia & Cricetidae & Holochilus & brasiliensis & Brazil & 2,59 & 0,00 & NA-SA \\
\hline 139916 & C. Jaramillo & Rodentia & Cricetidae & Holochilus & brasiliensis & Uruguay & 0,13 & 0,01 & NA-SA \\
\hline 63515 & C. Bell & Rodentia & Cricetidae & Holochilus & sciureus & Venezuela & 0,13 & 0,01 & NA-SA \\
\hline 71303 & J. Alroy & Rodentia & Cricetidae & Holochilus & brasiliensis & Argentina & 0,78 & 0,01 & NA-SA \\
\hline 71324 & J. Alroy & Rodentia & Cricetidae & Holochilus & chacarius & Bolivia & 0,01 & 0,01 & NA-SA \\
\hline 142338 & C. Jaramillo & Rodentia & Cricetidae & Holochilus & primigenus & Bolivia & 2,59 & 0,78 & NA-SA \\
\hline Castro et al. 2014 & M. Vallejo & Rodentia & Cricetidae & Holochilus & sciureus & Brazil & 0,13 & 0,01 & NA-SA \\
\hline 71274 & J. Alroy & Rodentia & Cricetidae & Holochilus & sp. & Uruguay & 0,78 & 0,01 & NA-SA \\
\hline 70673 & D. Croft & Rodentia & Cricetidae & Kunsia & fronto & Bolivia & 2,59 & 0,78 & NA-SA \\
\hline 92813 & J. Alroy & Rodentia & Cricetidae & Kunsia & tomentosus & Brazil & 2,59 & 0,00 & NA-SA \\
\hline Pino et al. 2013 & M. Vallejo & Rodentia & Cricetidae & Loxodontomys & micropus & Chile & 0,01 & 0,01 & NA-SA \\
\hline 63335 & D. Croft & Rodentia & Cricetidae & Lundomys & sp. & Argentina & 0,78 & 0,13 & NA-SA \\
\hline 152062 & C. Jaramillo & Rodentia & Cricetidae & Necromys & bonapartei & Argentina & 6,80 & 4,00 & NA-SA \\
\hline 152081 & C. Jaramillo & Rodentia & Cricetidae & Necromys & bonapartei & Argentina & 11,61 & 3,60 & NA-SA \\
\hline 141182 & C. Jaramillo & Rodentia & Cricetidae & Neotomys & ebriosus & Argentina & 0,03 & 0,02 & NA-SA \\
\hline 71324 & J. Alroy & Rodentia & Cricetidae & Necromys & lactens & Bolivia & 0,01 & 0,01 & NA-SA \\
\hline 141182 & C. Jaramillo & Rodentia & Cricetidae & Necromys & lactens & Argentina & 0,03 & 0,02 & NA-SA \\
\hline 70673 & D. Croft & Rodentia & Cricetidae & Nectomys & squamipes & Bolivia & 2,59 & 0,78 & NA-SA \\
\hline 141182 & C. Jaramillo & Rodentia & Cricetidae & Oligoryzomys & flavescens & Argentina & 0,03 & 0,02 & NA-SA \\
\hline 141182 & C. Jaramillo & Rodentia & Cricetidae & Oligoryzomys & longicaudatus & Argentina & 0,03 & 0,02 & NA-SA \\
\hline 92813 & J. Alroy & Rodentia & Cricetidae & Oligoryzomys & sp. & Brazil & 2,59 & 0,00 & NA-SA \\
\hline 63335 & D. Croft & Rodentia & Cricetidae & Oxymycterus & sp. & Argentina & 0,78 & 0,13 & NA-SA \\
\hline 70673 & D. Croft & Rodentia & Cricetidae & Oxymycterus & paranensis & Bolivia & 2,59 & 0,78 & NA-SA \\
\hline 141182 & C. Jaramillo & Rodentia & Cricetidae & Oxymycterus & paramensis & Argentina & 0,03 & 0,02 & NA-SA \\
\hline 70673 & D. Croft & Rodentia & Cricetidae & Phyllotis & darwini & Bolivia & 2,59 & 0,78 & NA-SA \\
\hline 63335 & D. Croft & Rodentia & Cricetidae & Phyllotis & sp. & Argentina & 0,78 & 0,13 & NA-SA \\
\hline 71265 & J. Alroy & Rodentia & Cricetidae & Phyllotis & sp. & Chile & 0,13 & 0,01 & NA-SA \\
\hline 71266 & J. Alroy & Rodentia & Cricetidae & Phyllotis & sp. & Chile & 0,01 & 0,01 & NA-SA \\
\hline 71324 & J. Alroy & Rodentia & Cricetidae & Phyllotis & sp. & Bolivia & 0,01 & 0,01 & NA-SA \\
\hline 71346 & J. Alroy & Rodentia & Cricetidae & Phyllotis & sp. & Peru & 0,13 & 0,01 & NA-SA \\
\hline 141182 & C. Jaramillo & Rodentia & Cricetidae & Phyllotis & sp. & Argentina & 0,03 & 0,02 & NA-SA \\
\hline 145500 & C. Jaramillo & Rodentia & Cricetidae & Phyllotis & sp. & Peru & 0,01 & 0,01 & NA-SA \\
\hline 146602 & C. Jaramillo & Rodentia & Cricetidae & Phyllotis & sp. & Peru & 0,02 & 0,01 & NA-SA \\
\hline 63335 & D. Croft & Rodentia & Cricetidae & Reithrodon & auritus & Argentina & 0,78 & 0,13 & NA-SA \\
\hline 63338 & J. Alroy & Rodentia & Cricetidae & Reithrodon & auritus & Argentina & 0,01 & 0,00 & NA-SA \\
\hline 71303 & J. Alroy & Rodentia & Cricetidae & Reithrodon & auritus & Argentina & 0,78 & 0,01 & NA-SA \\
\hline 71330 & J. Alroy & Rodentia & Cricetidae & Reithrodon & auritus & Argentina & 0,13 & 0,01 & NA-SA \\
\hline 71347 & J. Alroy & Rodentia & Cricetidae & Reithrodon & auritus & Argentina & 0,78 & 0,01 & NA-SA \\
\hline
\end{tabular}




\begin{tabular}{|c|c|c|c|c|c|c|c|c|c|}
\hline 73845 & J. Alroy & Rodentia & Cricetidae & Reithrodon & auritus & Argentina & 0,13 & 0,01 & NA-SA \\
\hline 140805 & C. Jaramillo & Rodentia & Cricetidae & Reithrodon & auritus & Argentina & 0,78 & 0,01 & NA-SA \\
\hline 140807 & C. Jaramillo & Rodentia & Cricetidae & Reithrodon & auritus & Argentina & 0,78 & 0,01 & NA-SA \\
\hline 140934 & C. Jaramillo & Rodentia & Cricetidae & Reithrodon & auritus & Argentina & 0,78 & 0,01 & NA-SA \\
\hline 141182 & C. Jaramillo & Rodentia & Cricetidae & Reithrodon & auritus & Argentina & 0,03 & 0,02 & NA-SA \\
\hline 145810 & C. Jaramillo & Rodentia & Cricetidae & Reithrodon & auritus & Argentina & 0,01 & 0,00 & NA-SA \\
\hline 140934 & C. Jaramillo & Rodentia & Cricetidae & Reithrodon & physodes & Argentina & 0,78 & 0,01 & NA-SA \\
\hline 71336 & J. Alroy & Rodentia & Cricetidae & Reithrodon & physodes & Argentina & 0,13 & 0,01 & NA-SA \\
\hline 92813 & J. Alroy & Rodentia & Cricetidae & Scapteromys & tumidus & Brazil & 2,59 & 0,00 & NA-SA \\
\hline 63515 & C. Bell & Rodentia & Cricetidae & Sigmodon & hispidus & Venezuela & 0,13 & 0,01 & NA-SA \\
\hline 71346 & J. Alroy & Rodentia & Cricetidae & Sigmodon & sp. & Peru & 0,13 & 0,01 & NA-SA \\
\hline 145500 & C. Jaramillo & Rodentia & Cricetidae & Sigmodon & sp. & Peru & 0,01 & 0,01 & NA-SA \\
\hline 141182 & C. Jaramillo & Rodentia & Cricetidae & Tafimys & powelli & Argentina & 0,03 & 0,02 & NA-SA \\
\hline 70705 & D. Croft & Rodentia & Cricetidae & Zygodontomys & lasiurus & Bolivia & 0,01 & 0,00 & NA-SA \\
\hline 71324 & J. Alroy & Rodentia & Cricetidae & Zygodontomys & sp. & Bolivia & 0,01 & 0,01 & NA-SA \\
\hline 19650 & J. Alroy & Rodentia & Erethizontidae & Erethizon & bathygnathum & United States & 4,90 & 1,80 & SA-NA \\
\hline 19913 & J. Alroy & Rodentia & Erethizontidae & Erethizon & bathygnathum & United States & 4,90 & 1,80 & SA-NA \\
\hline 20115 & J. Alroy & Rodentia & Erethizontidae & Erethizon & bathygnathum & United States & 4,90 & 1,80 & SA-NA \\
\hline 20118 & J. Alroy & Rodentia & Erethizontidae & Erethizon & bathygnathum & United States & 4,90 & 1,80 & SA-NA \\
\hline 20120 & J. Alroy & Rodentia & Erethizontidae & Erethizon & bathygnathum & United States & 4,90 & 1,80 & SA-NA \\
\hline 19737 & J. Alroy & Rodentia & Erethizontidae & Erethizon & cascoensis & United States & 4,90 & 1,80 & SA-NA \\
\hline 20319 & J. Alroy & Rodentia & Erethizontidae & Erethizon & cascoensis & United States & 1,80 & 0,30 & SA-NA \\
\hline 20145 & J. Alroy & Rodentia & Erethizontidae & Erethizon & dorsatum & United States & 1,80 & 0,30 & SA-NA \\
\hline 20283 & J. Alroy & Rodentia & Erethizontidae & Erethizon & dorsatum & Mexico & 1,80 & 0,30 & SA-NA \\
\hline 20289 & J. Alroy & Rodentia & Erethizontidae & Erethizon & dorsatum & United States & 1,80 & 0,30 & SA-NA \\
\hline 20290 & J. Alroy & Rodentia & Erethizontidae & Erethizon & dorsatum & United States & 1,80 & 0,30 & SA-NA \\
\hline 20302 & J. Alroy & Rodentia & Erethizontidae & Erethizon & dorsatum & United States & 1,80 & 0,30 & SA-NA \\
\hline 20350 & J. Alroy & Rodentia & Erethizontidae & Erethizon & dorsatum & United States & 1,80 & 0,30 & SA-NA \\
\hline 20351 & J. Alroy & Rodentia & Erethizontidae & Erethizon & dorsatum & United States & 1,80 & 0,30 & SA-NA \\
\hline 20354 & J. Alroy & Rodentia & Erethizontidae & Erethizon & dorsatum & United States & 1,80 & 0,30 & SA-NA \\
\hline 20479 & J. Alroy & Rodentia & Erethizontidae & Erethizon & dorsatum & United States & 1,80 & 0,30 & SA-NA \\
\hline 20554 & J. Alroy & Rodentia & Erethizontidae & Erethizon & dorsatum & United States & 1,80 & 0,30 & SA-NA \\
\hline 20569 & J. Alroy & Rodentia & Erethizontidae & Erethizon & dorsatum & United States & 0,13 & 0,01 & SA-NA \\
\hline 20615 & J. Alroy & Rodentia & Erethizontidae & Erethizon & dorsatum & Canada & 0,13 & 0,01 & SA-NA \\
\hline 20641 & J. Alroy & Rodentia & Erethizontidae & Erethizon & dorsatum & United States & 0,13 & 0,01 & SA-NA \\
\hline 63565 & C. Bell & Rodentia & Erethizontidae & Erethizon & dorsatum & United States & 0,13 & 0,00 & SA-NA \\
\hline 63571 & C. Bell & Rodentia & Erethizontidae & Erethizon & dorsatum & United States & 0,13 & 0,00 & SA-NA \\
\hline 71345 & J. Alroy & Rodentia & Erethizontidae & Erethizon & dorsatum & Mexico & 0,13 & 0,01 & SA-NA \\
\hline 74235 & J. Alroy & Rodentia & Erethizontidae & Erethizon & dorsatum & United States & 0,13 & 0,01 & SA-NA \\
\hline 74246 & J. Alroy & Rodentia & Erethizontidae & Erethizon & dorsatum & United States & 2,59 & 0,01 & SA-NA \\
\hline 74473 & J. Alroy & Rodentia & Erethizontidae & Erethizon & dorsatum & United States & 0,13 & 0,01 & SA-NA \\
\hline 76059 & J. Alroy & Rodentia & Erethizontidae & Erethizon & dorsatum & United States & 0,03 & 0,03 & SA-NA \\
\hline 79660 & J. Alroy & Rodentia & Erethizontidae & Erethizon & dorsatum & United States & 2,59 & 0,00 & SA-NA \\
\hline 79757 & M. Uhen & Rodentia & Erethizontidae & Erethizon & dorsatum & United States & 0,30 & 0,01 & SA-NA \\
\hline 82258 & M. Uhen & Rodentia & Erethizontidae & Erethizon & dorsatum & United States & 0,13 & 0,01 & SA-NA \\
\hline 93141 & J. Alroy & Rodentia & Erethizontidae & Erethizon & dorsatum & United States & 0,13 & 0,01 & SA-NA \\
\hline 93179 & J. Alroy & Rodentia & Erethizontidae & Erethizon & dorsatum & United States & 0,13 & 0,01 & SA-NA \\
\hline 93250 & J. Alroy & Rodentia & Erethizontidae & Erethizon & dorsatum & United States & 0,13 & 0,01 & SA-NA \\
\hline 93258 & J. Alroy & Rodentia & Erethizontidae & Erethizon & dorsatum & United States & 2,59 & 0,00 & SA-NA \\
\hline 93332 & J. Alroy & Rodentia & Erethizontidae & Erethizon & dorsatum & United States & 0,03 & 0,03 & SA-NA \\
\hline 93334 & J. Alroy & Rodentia & Erethizontidae & Erethizon & dorsatum & United States & 0,13 & 0,01 & SA-NA \\
\hline 103991 & M. Uhen & Rodentia & Erethizontidae & Erethizon & dorsatum & United States & 0,13 & 0,01 & SA-NA \\
\hline 104044 & M. Uhen & Rodentia & Erethizontidae & Erethizon & dorsatum & United States & 0,13 & 0,00 & SA-NA \\
\hline 104672 & M. Uhen & Rodentia & Erethizontidae & Erethizon & dorsatum & United States & 0,13 & 0,00 & SA-NA \\
\hline 104697 & M. Uhen & Rodentia & Erethizontidae & Erethizon & dorsatum & United States & 0,03 & 0,03 & SA-NA \\
\hline 104758 & M. Uhen & Rodentia & Erethizontidae & Erethizon & dorsatum & United States & 0,04 & 0,01 & SA-NA \\
\hline 123868 & M. Uhen & Rodentia & Erethizontidae & Erethizon & dorsatum & United States & 0,13 & 0,01 & SA-NA \\
\hline 124198 & M. Uhen & Rodentia & Erethizontidae & Erethizon & dorsatum & United States & 0,13 & 0,01 & SA-NA \\
\hline 124199 & M. Uhen & Rodentia & Erethizontidae & Erethizon & dorsatum & United States & 0,13 & 0,01 & SA-NA \\
\hline 124319 & M. Uhen & Rodentia & Erethizontidae & Erethizon & dorsatum & United States & 2,59 & 0,01 & SA-NA \\
\hline 124709 & M. Uhen & Rodentia & Erethizontidae & Erethizon & dorsatum & United States & 2,59 & 0,01 & SA-NA \\
\hline 125941 & M. Uhen & Rodentia & Erethizontidae & Erethizon & dorsatum & United States & 2,59 & 0,01 & SA-NA \\
\hline 126660 & M. Uhen & Rodentia & Erethizontidae & Erethizon & dorsatum & United States & 2,59 & 0,01 & SA-NA \\
\hline 20384 & J. Alroy & Rodentia & Erethizontidae & Erethizon & kleini & United States & 4,90 & 1,80 & SA-NA \\
\hline 20419 & J. Alroy & Rodentia & Erethizontidae & Erethizon & kleini & United States & 1,80 & 0,01 & SA-NA \\
\hline 19750 & J. Alroy & Rodentia & Erethizontidae & Erethizon & poyeri & United States & 4,90 & 0,30 & SA-NA \\
\hline 98313 & M. Uhen & Rodentia & Erethizontidae & Erethizon & poyeri & United States & 4,90 & 0,30 & SA-NA \\
\hline 20117 & J. Alroy & Rodentia & Erethizontidae & Erethizon & sp. & United States & 4,90 & 1,80 & SA-NA \\
\hline 20121 & J. Alroy & Rodentia & Erethizontidae & Erethizon & sp. & United States & 4,90 & 1,80 & SA-NA \\
\hline 20126 & J. Alroy & Rodentia & Erethizontidae & Erethizon & sp. & United States & 1,80 & 0,30 & SA-NA \\
\hline 20138 & J. Alroy & Rodentia & Erethizontidae & Erethizon & sp. & United States & 1,80 & 0,30 & SA-NA \\
\hline 20187 & J. Alroy & Rodentia & Erethizontidae & Erethizon & sp. & United States & $\begin{array}{l}1,00 \\
1,80\end{array}$ & 0,30 & SA-NA \\
\hline 20302 & J. Alroy & Rodentia & Erethizontidae & Erethizon & sp. & United States & 1,80 & 0,30 & SA-NA \\
\hline 20477 & J. Alroy & Rodentia & Erethizontidae & Erethizon & sp. & United States & 1,80 & 0,30 & SA-NA \\
\hline 20504 & J. Alroy & Rodentia & Erethizontidae & Erethizon & sp. & United States & 1,80 & 0,30 & SA-NA \\
\hline 20514 & J. Alroy & Rodentia & Erethizontidae & Erethizon & sp. & United States & 1,80 & 0,30 & SA-NA \\
\hline 20559 & J. Alroy & Rodentia & Erethizontidae & Erethizon & sp. & United States & 1,80 & 0,30 & SA-NA \\
\hline 28221 & J. Alroy & Rodentia & Erethizontidae & Erethizon & sp. & United States & 1,80 & 0,30 & SA-NA \\
\hline 145810 & C. Jaramillo & Rodentia & Muridae & Bolomys & obscurus & Argentina & 0,01 & 0,00 & NA-SA \\
\hline
\end{tabular}




\begin{tabular}{|c|c|c|c|c|c|c|c|c|c|}
\hline 92813 & J. Alroy & Rodentia & Muridae & Bolomys & sp. & Brazil & 2,59 & 0,00 & NA-SA \\
\hline 71330 & J. Alroy & Rodentia & Muridae & Colomys & sp. & Argentina & 0,13 & 0,01 & NA-SA \\
\hline 63515 & C. Bell & Rodentia & Muridae & Heteromys & anomalus & Venezuela & 0,13 & 0,01 & NA-SA \\
\hline 71291 & J. Alroy & Soricomorpha & Soricidae & Cryptotis & sp. & Colombia & 0,78 & 0,01 & NA-SA \\
\hline 13066 & M. Uhen & Xenarthra & Dasypodidae & Dasypus & bellus & United States & 2,59 & 0,01 & SA-NA \\
\hline 19750 & J. Alroy & Xenarthra & Dasypodidae & Dasypus & bellus & United States & 4,90 & 0,30 & SA-NA \\
\hline 19752 & J. Alroy & Xenarthra & Dasypodidae & Dasypus & bellus & United States & 4,90 & 1,80 & SA-NA \\
\hline 19753 & J. Alroy & Xenarthra & Dasypodidae & Dasypus & bellus & United States & 4,90 & 1,80 & SA-NA \\
\hline 19754 & J. Alroy & Xenarthra & Dasypodidae & Dasypus & bellus & United States & 4,90 & 1,80 & SA-NA \\
\hline 19758 & J. Alroy & Xenarthra & Dasypodidae & Dasypus & bellus & United States & 4,90 & 1,80 & SA-NA \\
\hline 19954 & J. Alroy & Xenarthra & Dasypodidae & Dasypus & bellus & United States & 4,90 & 1,80 & SA-NA \\
\hline 20151 & J. Alroy & Xenarthra & Dasypodidae & Dasypus & bellus & United States & 1,80 & 0,30 & SA-NA \\
\hline 20281 & J. Alroy & Xenarthra & Dasypodidae & Dasypus & bellus & United States & 1,80 & 0,30 & SA-NA \\
\hline 20289 & J. Alroy & Xenarthra & Dasypodidae & Dasypus & bellus & United States & 1,80 & 0,30 & SA-NA \\
\hline 20308 & J. Alroy & Xenarthra & Dasypodidae & Dasypus & bellus & United States & 1,80 & 0,30 & SA-NA \\
\hline 20318 & J. Alroy & Xenarthra & Dasypodidae & Dasypus & bellus & United States & 1,80 & 0,30 & SA-NA \\
\hline 20350 & J. Alroy & Xenarthra & Dasypodidae & Dasypus & bellus & United States & 1,80 & 0,30 & SA-NA \\
\hline 20351 & J. Alroy & Xenarthra & Dasypodidae & Dasypus & bellus & United States & 1,80 & 0,30 & SA-NA \\
\hline 20384 & J. Alroy & Xenarthra & Dasypodidae & Dasypus & bellus & United States & 4,90 & 1,80 & SA-NA \\
\hline 20385 & J. Alroy & Xenarthra & Dasypodidae & Dasypus & bellus & United States & 1,80 & 0,30 & SA-NA \\
\hline 20400 & J. Alroy & Xenarthra & Dasypodidae & Dasypus & bellus & United States & 1,80 & 0,30 & SA-NA \\
\hline 20402 & J. Alroy & Xenarthra & Dasypodidae & Dasypus & bellus & United States & 1,80 & 0,30 & SA-NA \\
\hline 20403 & J. Alroy & Xenarthra & Dasypodidae & Dasypus & bellus & United States & 1,80 & 0,30 & SA-NA \\
\hline 20415 & J. Alroy & Xenarthra & Dasypodidae & Dasypus & bellus & United States & 1,80 & 0,30 & SA-NA \\
\hline 20440 & J. Alroy & Xenarthra & Dasypodidae & Dasypus & bellus & United States & 0,30 & 0,01 & SA-NA \\
\hline 20475 & J. Alroy & Xenarthra & Dasypodidae & Dasypus & bellus & United States & 1,80 & 0,30 & SA-NA \\
\hline 20491 & J. Alroy & Xenarthra & Dasypodidae & Dasypus & bellus & United States & 0,30 & 0,01 & SA-NA \\
\hline 20494 & J. Alroy & Xenarthra & Dasypodidae & Dasypus & bellus & United States & 1,80 & 0,30 & SA-NA \\
\hline 20520 & J. Alroy & Xenarthra & Dasypodidae & Dasypus & bellus & United States & 1,80 & 0,30 & SA-NA \\
\hline 20530 & J. Alroy & Xenarthra & Dasypodidae & Dasypus & bellus & United States & 1,80 & 0,30 & SA-NA \\
\hline 20572 & J. Alroy & Xenarthra & Dasypodidae & Dasypus & bellus & United States & 0,13 & 0,01 & SA-NA \\
\hline 20573 & J. Alroy & Xenarthra & Dasypodidae & Dasypus & bellus & United States & 0,13 & 0,01 & SA-NA \\
\hline 20584 & J. Alroy & Xenarthra & Dasypodidae & Dasypus & bellus & United States & 0,13 & 0,01 & SA-NA \\
\hline 20593 & J. Alroy & Xenarthra & Dasypodidae & Dasypus & bellus & United States & 0,13 & 0,01 & SA-NA \\
\hline 20594 & J. Alroy & Xenarthra & Dasypodidae & Dasypus & bellus & United States & 0,13 & 0,01 & SA-NA \\
\hline 20595 & J. Alroy & Xenarthra & Dasypodidae & Dasypus & bellus & United States & 0,13 & 0,01 & SA-NA \\
\hline 20598 & J. Alroy & Xenarthra & Dasypodidae & Dasypus & bellus & United States & 0,13 & 0,01 & SA-NA \\
\hline 20604 & J. Alroy & Xenarthra & Dasypodidae & Dasypus & bellus & United States & 0,13 & 0,01 & SA-NA \\
\hline 20608 & J. Alroy & Xenarthra & Dasypodidae & Dasypus & bellus & United States & 0,13 & 0,01 & SA-NA \\
\hline 20613 & J. Alroy & Xenarthra & Dasypodidae & Dasypus & bellus & United States & 0,13 & 0,01 & SA-NA \\
\hline 20634 & J. Alroy & Xenarthra & Dasypodidae & Dasypus & bellus & United States & 0,13 & 0,01 & SA-NA \\
\hline 20636 & J. Alroy & Xenarthra & Dasypodidae & Dasypus & bellus & United States & 0,13 & 0,01 & SA-NA \\
\hline 20638 & J. Alroy & Xenarthra & Dasypodidae & Dasypus & bellus & United States & 0,13 & 0,01 & SA-NA \\
\hline 20650 & J. Alroy & Xenarthra & Dasypodidae & Dasypus & bellus & United States & 0,13 & 0,01 & SA-NA \\
\hline 20653 & J. Alroy & Xenarthra & Dasypodidae & Dasypus & bellus & United States & 0,13 & 0,01 & SA-NA \\
\hline 58089 & M. Uhen & Xenarthra & Dasypodidae & Dasypus & bellus & United States & 1,80 & 0,30 & SA-NA \\
\hline 63563 & C. Bell & Xenarthra & Dasypodidae & Dasypus & bellus & United States & 0,13 & 0,00 & SA-NA \\
\hline 63565 & C. Bell & Xenarthra & Dasypodidae & Dasypus & bellus & United States & 0,13 & 0,00 & SA-NA \\
\hline 63569 & C. Bell & Xenarthra & Dasypodidae & Dasypus & bellus & United States & 0,13 & 0,00 & SA-NA \\
\hline 63570 & C. Bell & Xenarthra & Dasypodidae & Dasypus & bellus & United States & 0,13 & 0,00 & SA-NA \\
\hline 65405 & M. Uhen & Xenarthra & Dasypodidae & Dasypus & bellus & United States & 2,59 & 0,01 & SA-NA \\
\hline 75974 & J. Alroy & Xenarthra & Dasypodidae & Dasypus & bellus & United States & 0,78 & 0,13 & SA-NA \\
\hline 75995 & J. Alroy & Xenarthra & Dasypodidae & Dasypus & bellus & United States & 0,13 & 0,01 & SA-NA \\
\hline 79667 & J. Alroy & Xenarthra & Dasypodidae & Dasypus & bellus & United States & 0,13 & 0,01 & SA-NA \\
\hline 79759 & M. Uhen & Xenarthra & Dasypodidae & Dasypus & bellus & United States & 1,80 & 0,30 & SA-NA \\
\hline 79760 & M. Uhen & Xenarthra & Dasypodidae & Dasypus & bellus & United States & 0,30 & 0,01 & SA-NA \\
\hline 84189 & M. Uhen & Xenarthra & Dasypodidae & Dasypus & bellus & United States & 0,13 & 0,01 & SA-NA \\
\hline 93103 & J. Alroy & Xenarthra & Dasypodidae & Dasypus & bellus & United States & 0,13 & 0,01 & SA-NA \\
\hline 98313 & M. Uhen & Xenarthra & Dasypodidae & Dasypus & bellus & United States & 4,90 & 0,30 & SA-NA \\
\hline 104681 & M. Uhen & Xenarthra & Dasypodidae & Dasypus & bellus & United States & 0,13 & 0,01 & SA-NA \\
\hline 113275 & J. Alroy & Xenarthra & Dasypodidae & Dasypus & bellus & United States & 4,90 & 1,80 & SA-NA \\
\hline 113276 & J. Alroy & Xenarthra & Dasypodidae & Dasypus & bellus & United States & 4,90 & 1,80 & SA-NA \\
\hline 34269 & M. Carrano & Xenarthra & Dasypodidae & Dasypus & sp. & United States & 0,13 & 0,01 & SA-NA \\
\hline 71312 & J. Alroy & Xenarthra & Dasypodidae & Dasypus & sp. & Guatemala & 0,13 & 0,01 & SA-NA \\
\hline 71278 & J. Alroy & Xenarthra & Glyptodontidae & Lomaphorus & sp. & Panama & 0,13 & 0,01 & SA-NA \\
\hline 71278 & J. Alroy & Xenarthra & Glyptodontidae & Glyptodon & sp. & Panama & 0,13 & 0,01 & SA-NA \\
\hline 71312 & J. Alroy & Xenarthra & Glyptodontidae & Glyptodon & sp. & Guatemala & 0,13 & 0,01 & SA-NA \\
\hline 19646 & J. Alroy & Xenarthra & Glyptodontidae & Glyptotherium & arizonae & United States & 4,90 & 1,80 & SA-NA \\
\hline 19655 & J. Alroy & Xenarthra & Glyptodontidae & Glyptotherium & arizonae & United States & 4,90 & 1,80 & SA-NA \\
\hline 19747 & J. Alroy & Xenarthra & Glyptodontidae & Glyptotherium & arizonae & United States & 4,90 & 1,80 & SA-NA \\
\hline 19750 & J. Alroy & Xenarthra & Glyptodontidae & Glyptotherium & arizonae & United States & 4,90 & 0,30 & SA-NA \\
\hline 19754 & J. Alroy & Xenarthra & Glyptodontidae & Glyptotherium & arizonae & United States & 4,90 & 1,80 & SA-NA \\
\hline 19758 & J. Alroy & Xenarthra & Glyptodontidae & Glyptotherium & arizonae & United States & 4,90 & 1,80 & SA-NA \\
\hline 19764 & J. Alroy & Xenarthra & Glyptodontidae & Glyptotherium & arizonae & United States & 4,90 & 1,80 & SA-NA \\
\hline 20077 & J. Alroy & Xenarthra & Glyptodontidae & Glyptotherium & arizonae & Mexico & 4,90 & 1,80 & SA-NA \\
\hline 20141 & J. Alroy & Xenarthra & Glyptodontidae & Glyptotherium & arizonae & United States & 4,90 & 0,30 & SA-NA \\
\hline 20308 & J. Alroy & Xenarthra & Glyptodontidae & Glyptotherium & arizonae & United States & 1,80 & 0,30 & SA-NA \\
\hline 20341 & J. Alroy & Xenarthra & Glyptodontidae & Glyptotherium & arizonae & United States & 1,80 & 0,30 & SA-NA \\
\hline 20342 & J. Alroy & Xenarthra & Glyptodontidae & Glyptotherium & arizonae & United States & 1,80 & 0,30 & SA-NA \\
\hline
\end{tabular}




\begin{tabular}{|c|c|c|c|c|c|c|c|c|c|}
\hline 20344 & J. Alroy & Xenarthra & Glyptodontidae & Glyptotherium & arizonae & United States & 1,80 & 0,30 & SA-NA \\
\hline 20382 & J. Alroy & Xenarthra & Glyptodontidae & Glyptotherium & arizonae & United States & 1,80 & 0,30 & SA-NA \\
\hline 20384 & J. Alroy & Xenarthra & Glyptodontidae & Glyptotherium & arizonae & United States & 4,90 & 1,80 & SA-NA \\
\hline 20399 & J. Alroy & Xenarthra & Glyptodontidae & Glyptotherium & arizonae & United States & 1,80 & 0,30 & SA-NA \\
\hline 20412 & J. Alroy & Xenarthra & Glyptodontidae & Glyptotherium & arizonae & United States & 1,80 & 0,30 & SA-NA \\
\hline 20548 & J. Alroy & Xenarthra & Glyptodontidae & Glyptotherium & arizonae & United States & 1,80 & 0,30 & SA-NA \\
\hline 70118 & J. Alroy & Xenarthra & Glyptodontidae & Glyptotherium & arizonae & El Salvador & 2,59 & 0,13 & SA-NA \\
\hline 98313 & M. Uhen & Xenarthra & Glyptodontidae & Glyptotherium & arizonae & United States & 4,90 & 0,30 & SA-NA \\
\hline 150272 & M. Uhen & Xenarthra & Glyptodontidae & Glyptotherium & arizonae & United States & 5,33 & 0,01 & SA-NA \\
\hline Perez 2013 & M. Vallejo & Xenarthra & Glyptodontidae & Glyptotherium & arizonae & Costa Rica & 1,80 & 0,30 & SA-NA \\
\hline 20495 & J. Alroy & Xenarthra & Glyptodontidae & Glyptotherium & cylindricum & Mexico & 0,30 & 0,01 & SA-NA \\
\hline 85156 & J. Alroy & Xenarthra & Glyptodontidae & Glyptotherium & cylindricum & Mexico & 0,13 & 0,01 & SA-NA \\
\hline 85159 & J. Alroy & Xenarthra & Glyptodontidae & Glyptotherium & cylindricum & Mexico & 0,13 & 0,01 & SA-NA \\
\hline 20520 & J. Alroy & Xenarthra & Glyptodontidae & Glyptotherium & floridanum & United States & 1,80 & 0,30 & SA-NA \\
\hline 65405 & M. Uhen & Xenarthra & Glyptodontidae & Glyptotherium & floridanum & United States & 2,59 & 0,01 & SA-NA \\
\hline 113408 & M. Uhen & Xenarthra & Glyptodontidae & Glyptotherium & floridanum & Mexico & 0,30 & 0,01 & SA-NA \\
\hline 113409 & M. Uhen & Xenarthra & Glyptodontidae & Glyptotherium & floridanum & Mexico & 0,30 & 0,01 & SA-NA \\
\hline 113410 & M. Uhen & Xenarthra & Glyptodontidae & Glyptotherium & floridanum & Mexico & 0,30 & 0,01 & SA-NA \\
\hline 124936 & M. Uhen & Xenarthra & Glyptodontidae & Glyptotherium & floridanum & United States & 2,59 & 0,01 & SA-NA \\
\hline Lucas 2014 & M. Vallejo & Xenarthra & Glyptodontidae & Glyptotherium & floridanum & Panama & 0,47 & 0,45 & SA-NA \\
\hline 20283 & J. Alroy & Xenarthra & Glyptodontidae & Glyptotherium & mexicanum & Mexico & 1,80 & 0,30 & SA-NA \\
\hline 71319 & J. Alroy & Xenarthra & Glyptodontidae & Glyptotherium & mexicanum & Mexico & 2,59 & 0,01 & SA-NA \\
\hline 71320 & J. Alroy & Xenarthra & Glyptodontidae & Glyptotherium & mexicanum & Mexico & 2,59 & 0,01 & SA-NA \\
\hline 19652 & J. Alroy & Xenarthra & Glyptodontidae & Glyptotherium & texanum & United States & 4,90 & 0,30 & SA-NA \\
\hline 19656 & J. Alroy & Xenarthra & Glyptodontidae & Glyptotherium & texanum & United States & 4,90 & 1,80 & SA-NA \\
\hline 19657 & J. Alroy & Xenarthra & Glyptodontidae & Glyptotherium & texanum & United States & 4,90 & 1,80 & SA-NA \\
\hline 19658 & J. Alroy & Xenarthra & Glyptodontidae & Glyptotherium & texanum & United States & 4,90 & 1,80 & SA-NA \\
\hline 20028 & J. Alroy & Xenarthra & Glyptodontidae & Glyptotherium & texanum & United States & 4,90 & 1,80 & SA-NA \\
\hline 20032 & J. Alroy & Xenarthra & Glyptodontidae & Glyptotherium & texanum & United States & 4,90 & 1,80 & SA-NA \\
\hline 20033 & J. Alroy & Xenarthra & Glyptodontidae & Glyptotherium & texanum & United States & 4,90 & 1,80 & SA-NA \\
\hline 20050 & J. Alroy & Xenarthra & Glyptodontidae & Glyptotherium & texanum & United States & 4,90 & 1,80 & SA-NA \\
\hline 20057 & J. Alroy & Xenarthra & Glyptodontidae & Glyptotherium & texanum & United States & 4,90 & 1,80 & SA-NA \\
\hline 113469 & M. Uhen & Xenarthra & Glyptodontidae & Glyptotherium & texanum & Costa Rica & 2,59 & 0,01 & SA-NA \\
\hline 20046 & J. Alroy & Xenarthra & Glyptodontidae & Glyptotherium & sp. & United States & 4,90 & 1,80 & SA-NA \\
\hline 20047 & J. Alroy & Xenarthra & Glyptodontidae & Glyptotherium & sp. & United States & 4,90 & 1,80 & SA-NA \\
\hline 20078 & J. Alroy & Xenarthra & Glyptodontidae & Glyptotherium & sp. & Mexico & 4,90 & 0,30 & SA-NA \\
\hline 20139 & J. Alroy & Xenarthra & Glyptodontidae & Glyptotherium & sp. & United States & 1,80 & 0,30 & SA-NA \\
\hline 20164 & J. Alroy & Xenarthra & Glyptodontidae & Glyptotherium & sp. & United States & 1,80 & 0,30 & SA-NA \\
\hline 20397 & J. Alroy & Xenarthra & Glyptodontidae & Glyptotherium & sp. & United States & 4,90 & 0,30 & SA-NA \\
\hline 20536 & J. Alroy & Xenarthra & Glyptodontidae & Glyptotherium & sp. & United States & 1,80 & 0,30 & SA-NA \\
\hline 20539 & J. Alroy & Xenarthra & Glyptodontidae & Glyptotherium & sp. & Mexico & 1,80 & 0,30 & SA-NA \\
\hline 70114 & J. Alroy & Xenarthra & Glyptodontidae & Glyptotherium & sp. & Mexico & 4,90 & 1,80 & SA-NA \\
\hline 70117 & J. Alroy & Xenarthra & Glyptodontidae & Glyptotherium & sp. & Mexico & 4,90 & 1,80 & SA-NA \\
\hline 74470 & J. Alroy & Xenarthra & Glyptodontidae & Glyptotherium & sp. & United States & 0,13 & 0,01 & SA-NA \\
\hline 74498 & J. Alroy & Xenarthra & Glyptodontidae & Glyptotherium & sp. & Mexico & 0,30 & 0,01 & SA-NA \\
\hline 93527 & J. Alroy & Xenarthra & Glyptodontidae & Glyptotherium & sp. & United States & 0,13 & 0,01 & SA-NA \\
\hline Tovar et al 2014 & M. Vallejo & Xenarthra & Glyptodontidae & Glyptotherium & sp. & Mexico & 0,03 & 0,03 & SA-NA \\
\hline Tovar et al 2014 & M. Vallejo & Xenarthra & Glyptodontidae & Glyptotherium & sp. & Mexico & 0,03 & 0,03 & SA-NA \\
\hline 18033 & J. Alroy & Xenarthra & Megalonychidae & Megalonyx & curvidens & United States & 10,30 & 4,90 & SA-NA \\
\hline 18126 & J. Alroy & Xenarthra & Megalonychidae & Megalonyx & curvidens & United States & 10,30 & 4,90 & SA-NA \\
\hline 18201 & J. Alroy & Xenarthra & Megalonychidae & Megalonyx & curvidens & United States & 10,30 & 4,90 & SA-NA \\
\hline 18247 & J. Alroy & Xenarthra & Megalonychidae & Megalonyx & curvidens & United States & 10,30 & 4,90 & SA-NA \\
\hline 18323 & J. Alroy & Xenarthra & Megalonychidae & Megalonyx & curvidens & United States & 10,30 & 4,90 & SA-NA \\
\hline 18359 & J. Alroy & Xenarthra & Megalonychidae & Megalonyx & curvidens & United States & 10,30 & 4,90 & SA-NA \\
\hline 18441 & J. Alroy & Xenarthra & Megalonychidae & Megalonyx & curvidens & United States & 10,30 & 4,90 & SA-NA \\
\hline 18499 & J. Alroy & Xenarthra & Megalonychidae & Megalonyx & curvidens & United States & 10,30 & 4,90 & SA-NA \\
\hline 20281 & J. Alroy & Xenarthra & Megalonychidae & Megalonyx & jeffersonii & United States & 1,80 & 0,30 & SA-NA \\
\hline 20310 & J. Alroy & Xenarthra & Megalonychidae & Megalonyx & jeffersonii & United States & 1,80 & 0,30 & SA-NA \\
\hline 20353 & J. Alroy & Xenarthra & Megalonychidae & Megalonyx & jeffersonii & United States & 1,80 & 0,30 & SA-NA \\
\hline 20382 & J. Alroy & Xenarthra & Megalonychidae & Megalonyx & jeffersonii & United States & 1,80 & 0,30 & SA-NA \\
\hline 20556 & J. Alroy & Xenarthra & Megalonychidae & Megalonyx & jeffersonii & United States & 1,80 & 0,30 & SA-NA \\
\hline 20569 & J. Alroy & Xenarthra & Megalonychidae & Megalonyx & jeffersonii & United States & 0,13 & 0,01 & SA-NA \\
\hline 20574 & J. Alroy & Xenarthra & Megalonychidae & Megalonyx & jeffersonii & United States & 0,13 & 0,01 & SA-NA \\
\hline 20584 & J. Alroy & Xenarthra & Megalonychidae & Megalonyx & jeffersonii & United States & 0,13 & 0,01 & SA-NA \\
\hline 20610 & J. Alroy & Xenarthra & Megalonychidae & Megalonyx & jeffersonii & Canada & 0,13 & 0,01 & SA-NA \\
\hline 20612 & J. Alroy & Xenarthra & Megalonychidae & Megalonyx & jeffersonii & United States & 0,13 & 0,01 & SA-NA \\
\hline 20624 & J. Alroy & Xenarthra & Megalonychidae & Megalonyx & jeffersonii & United States & 0,30 & 0,01 & SA-NA \\
\hline 20626 & J. Alroy & Xenarthra & Megalonychidae & Megalonyx & jeffersonii & Canada & 0,13 & 0,01 & SA-NA \\
\hline 63563 & C. Bell & Xenarthra & Megalonychidae & Megalonyx & jeffersonii & United States & 0,13 & 0,00 & SA-NA \\
\hline 65405 & M. Uhen & Xenarthra & Megalonychidae & Megalonyx & jeffersonii & United States & 2,59 & 0,01 & SA-NA \\
\hline 79757 & M. Uhen & Xenarthra & Megalonychidae & Megalonyx & jeffersonii & United States & 0,30 & 0,01 & SA-NA \\
\hline 79760 & M. Uhen & Xenarthra & Megalonychidae & Megalonyx & jeffersonii & United States & 0,30 & 0,01 & SA-NA \\
\hline 79767 & M. Uhen & Xenarthra & Megalonychidae & Megalonyx & jeffersonii & United States & 0,78 & 0,13 & SA-NA \\
\hline 79768 & M. Uhen & Xenarthra & Megalonychidae & Megalonyx & jeffersonii & United States & 0,78 & 0,13 & SA-NA \\
\hline 79919 & M. Uhen & Xenarthra & Megalonychidae & Megalonyx & jeffersonii & United States & 1,80 & 0,30 & SA-NA \\
\hline 83073 & J. Alroy & Xenarthra & Megalonychidae & Megalonyx & jeffersonii & United States & 0,13 & 0,01 & SA-NA \\
\hline 85145 & J. Alroy & Xenarthra & Megalonychidae & Megalonyx & jeffersonii & United States & 2,59 & 0,78 & SA-NA \\
\hline 87059 & M. Uhen & Xenarthra & Megalonychidae & Megalonyx & jeffersonii & United States & 2,59 & 0,01 & SA-NA \\
\hline 93103 & J. Alroy & Xenarthra & Megalonychidae & Megalonyx & jeffersonii & United States & 0,13 & 0,01 & SA-NA \\
\hline
\end{tabular}




\begin{tabular}{|c|c|c|c|c|c|c|c|c|c|}
\hline 93208 & J. Alroy & Xenarthra & Megalonychidae & Megalonyx & jeffersonii & United States & 0,13 & 0,01 & SA-NA \\
\hline 93209 & J. Alroy & Xenarthra & Megalonychidae & Megalonyx & jeffersonii & United States & 0,13 & 0,01 & SA-NA \\
\hline 93266 & J. Alroy & Xenarthra & Megalonychidae & Megalonyx & jeffersonii & United States & 0,13 & 0,01 & SA-NA \\
\hline 93268 & J. Alroy & Xenarthra & Megalonychidae & Megalonyx & jeffersonii & United States & 0,13 & 0,01 & SA-NA \\
\hline 93379 & J. Alroy & Xenarthra & Megalonychidae & Megalonyx & jeffersonii & United States & 0,13 & 0,01 & SA-NA \\
\hline 93486 & J. Alroy & Xenarthra & Megalonychidae & Megalonyx & jeffersonii & United States & 0,13 & 0,01 & SA-NA \\
\hline 103358 & M. Uhen & Xenarthra & Megalonychidae & Megalonyx & jeffersonii & United States & 0,01 & 0,01 & SA-NA \\
\hline 104662 & M. Uhen & Xenarthra & Megalonychidae & Megalonyx & jeffersonii & United States & 0,78 & 0,13 & SA-NA \\
\hline 104678 & M. Uhen & Xenarthra & Megalonychidae & Megalonyx & jeffersonii & United States & 0,13 & 0,01 & SA-NA \\
\hline 108095 & M. Uhen & Xenarthra & Megalonychidae & Megalonyx & jeffersonii & United States & 2,59 & 0,01 & SA-NA \\
\hline Sertich et al 2014 & M. Vallejo & Xenarthra & Megalonychidae & Megalonyx & jeffersonii & United States & 0,14 & 0,08 & SA-NA \\
\hline 93332 & J. Alroy & Xenarthra & Megalonychidae & Megalonyx & jerrersonii & United States & 0,03 & 0,03 & SA-NA \\
\hline 19655 & J. Alroy & Xenarthra & Megalonychidae & Megalonyx & leptostomus & United States & 4,90 & 1,80 & SA-NA \\
\hline 19750 & J. Alroy & Xenarthra & Megalonychidae & Megalonyx & leptostomus & United States & 4,90 & 0,30 & SA-NA \\
\hline 19753 & J. Alroy & Xenarthra & Megalonychidae & Megalonyx & leptostomus & United States & 4,90 & 1,80 & SA-NA \\
\hline 19754 & J. Alroy & Xenarthra & Megalonychidae & Megalonyx & leptostomus & United States & 4,90 & 1,80 & SA-NA \\
\hline 19758 & J. Alroy & Xenarthra & Megalonychidae & Megalonyx & leptostomus & United States & 4,90 & 1,80 & SA-NA \\
\hline 19764 & J. Alroy & Xenarthra & Megalonychidae & Megalonyx & leptostomus & United States & 4,90 & 1,80 & SA-NA \\
\hline 19911 & J. Alroy & Xenarthra & Megalonychidae & Megalonyx & leptostomus & United States & 4,90 & 1,80 & SA-NA \\
\hline 19932 & J. Alroy & Xenarthra & Megalonychidae & Megalonyx & leptostomus & United States & 4,90 & 1,80 & SA-NA \\
\hline 19942 & J. Alroy & Xenarthra & Megalonychidae & Megalonyx & leptostomus & United States & 4,90 & 1,80 & SA-NA \\
\hline 19959 & J. Alroy & Xenarthra & Megalonychidae & Megalonyx & leptostomus & United States & 4,90 & 1,80 & SA-NA \\
\hline 19968 & J. Alroy & Xenarthra & Megalonychidae & Megalonyx & leptostomus & United States & 4,90 & 1,80 & SA-NA \\
\hline 20009 & J. Alroy & Xenarthra & Megalonychidae & Megalonyx & leptostomus & United States & 4,90 & 1,80 & SA-NA \\
\hline 20020 & J. Alroy & Xenarthra & Megalonychidae & Megalonyx & leptostomus & United States & 4,90 & 0,30 & SA-NA \\
\hline 20022 & J. Alroy & Xenarthra & Megalonychidae & Megalonyx & leptostomus & United States & 4,90 & 1,80 & SA-NA \\
\hline 20023 & J. Alroy & Xenarthra & Megalonychidae & Megalonyx & leptostomus & United States & 4,90 & 1,80 & SA-NA \\
\hline 20033 & J. Alroy & Xenarthra & Megalonychidae & Megalonyx & leptostomus & United States & 4,90 & 1,80 & SA-NA \\
\hline 20062 & J. Alroy & Xenarthra & Megalonychidae & Megalonyx & leptostomus & United States & 4,90 & 1,80 & SA-NA \\
\hline 20063 & J. Alroy & Xenarthra & Megalonychidae & Megalonyx & leptostomus & United States & 4,90 & 1,80 & SA-NA \\
\hline 20308 & J. Alroy & Xenarthra & Megalonychidae & Megalonyx & leptostomus & United States & 1,80 & 0,30 & SA-NA \\
\hline 20384 & J. Alroy & Xenarthra & Megalonychidae & Megalonyx & leptostomus & United States & 4,90 & 1,80 & SA-NA \\
\hline 98313 & M. Uhen & Xenarthra & Megalonychidae & Megalonyx & leptostomus & United States & 4,90 & 0,30 & SA-NA \\
\hline 19187 & J. Alroy & Xenarthra & Megalonychidae & Megalonyx & mathisi & United States & 10,30 & 4,90 & SA-NA \\
\hline 20152 & J. Alroy & Xenarthra & Megalonychidae & Megalonyx & obtusidens & El Salvador & 1,80 & 0,30 & SA-NA \\
\hline 20128 & J. Alroy & Xenarthra & Megalonychidae & Megalonyx & wheatleyi & United States & 1,80 & 0,30 & SA-NA \\
\hline 20158 & J. Alroy & Xenarthra & Megalonychidae & Megalonyx & wheatleyi & United States & 1,80 & 0,30 & SA-NA \\
\hline 20299 & J. Alroy & Xenarthra & Megalonychidae & Megalonyx & wheatleyi & United States & 1,80 & 0,30 & SA-NA \\
\hline 20322 & J. Alroy & Xenarthra & Megalonychidae & Megalonyx & wheatleyi & Mexico & 4,90 & 1,80 & SA-NA \\
\hline 20350 & J. Alroy & Xenarthra & Megalonychidae & Megalonyx & wheatleyi & United States & 1,80 & 0,30 & SA-NA \\
\hline 20397 & J. Alroy & Xenarthra & Megalonychidae & Megalonyx & wheatleyi & United States & 4,90 & 0,30 & SA-NA \\
\hline 20415 & J. Alroy & Xenarthra & Megalonychidae & Megalonyx & wheatleyi & United States & 1,80 & 0,30 & SA-NA \\
\hline 20475 & J. Alroy & Xenarthra & Megalonychidae & Megalonyx & wheatleyi & United States & 1,80 & 0,30 & SA-NA \\
\hline 20479 & J. Alroy & Xenarthra & Megalonychidae & Megalonyx & wheatleyi & United States & 1,80 & 0,30 & SA-NA \\
\hline 20638 & J. Alroy & Xenarthra & Megalonychidae & Megalonyx & wheatleyi & United States & 0,13 & 0,01 & SA-NA \\
\hline 18036 & J. Alroy & Xenarthra & Megalonychidae & Megalonyx & sp. & United States & 10,30 & 4,90 & SA-NA \\
\hline 18043 & J. Alroy & Xenarthra & Megalonychidae & Megalonyx & sp. & United States & 10,30 & 4,90 & SA-NA \\
\hline 18225 & J. Alroy & Xenarthra & Megalonychidae & Megalonyx & sp. & United States & 10,30 & 4,90 & SA-NA \\
\hline 18304 & J. Alroy & Xenarthra & Megalonychidae & Megalonyx & sp. & United States & 10,30 & 4,90 & SA-NA \\
\hline 18737 & J. Alroy & Xenarthra & Megalonychidae & Megalonyx & sp. & Mexico & 10,30 & 4,90 & SA-NA \\
\hline 18746 & J. Alroy & Xenarthra & Megalonychidae & Megalonyx & sp. & Mexico & 10,30 & 4,90 & SA-NA \\
\hline 19015 & J. Alroy & Xenarthra & Megalonychidae & Megalonyx & sp. & United States & 10,30 & 4,90 & SA-NA \\
\hline 19116 & J. Alroy & Xenarthra & Megalonychidae & Megalonyx & sp. & United States & 10,30 & 4,90 & SA-NA \\
\hline 19479 & J. Alroy & Xenarthra & Megalonychidae & Megalonyx & sp. & United States & 10,30 & 4,90 & SA-NA \\
\hline 19653 & J. Alroy & Xenarthra & Megalonychidae & Megalonyx & sp. & United States & 4,90 & 1,80 & SA-NA \\
\hline 19656 & J. Alroy & Xenarthra & Megalonychidae & Megalonyx & sp. & United States & 4,90 & 1,80 & SA-NA \\
\hline 19657 & J. Alroy & Xenarthra & Megalonychidae & Megalonyx & sp. & United States & 4,90 & 1,80 & SA-NA \\
\hline 19658 & J. Alroy & Xenarthra & Megalonychidae & Megalonyx & sp. & United States & 4,90 & 1,80 & SA-NA \\
\hline 19667 & J. Alroy & Xenarthra & Megalonychidae & Megalonyx & sp. & United States & 4,90 & 1,80 & SA-NA \\
\hline 19703 & J. Alroy & Xenarthra & Megalonychidae & Megalonyx & sp. & United States & 3,60 & 2,59 & SA-NA \\
\hline 19714 & J. Alroy & Xenarthra & Megalonychidae & Megalonyx & sp. & United States & 4,90 & 1,80 & SA-NA \\
\hline 19808 & J. Alroy & Xenarthra & Megalonychidae & Megalonyx & sp. & United States & 4,90 & 1,80 & SA-NA \\
\hline 19913 & J. Alroy & Xenarthra & Megalonychidae & Megalonyx & sp. & United States & 4,90 & 1,80 & SA-NA \\
\hline 19936 & J. Alroy & Xenarthra & Megalonychidae & Megalonyx & sp. & United States & 4,90 & 1,80 & SA-NA \\
\hline 19939 & J. Alroy & Xenarthra & Megalonychidae & Megalonyx & sp. & United States & 4,90 & 1,80 & SA-NA \\
\hline 19954 & J. Alroy & Xenarthra & Megalonychidae & Megalonyx & sp. & United States & 4,90 & 1,80 & SA-NA \\
\hline 19955 & J. Alroy & Xenarthra & Megalonychidae & Megalonyx & sp. & United States & 4,90 & 1,80 & SA-NA \\
\hline 20034 & J. Alroy & Xenarthra & Megalonychidae & Megalonyx & sp. & United States & 4,90 & 1,80 & SA-NA \\
\hline 20038 & J. Alroy & Xenarthra & Megalonychidae & Megalonyx & sp. & United States & 4,90 & 1,80 & SA-NA \\
\hline 20052 & J. Alroy & Xenarthra & Megalonychidae & Megalonyx & sp. & United States & 4,90 & 1,80 & SA-NA \\
\hline 20075 & J. Alroy & Xenarthra & Megalonychidae & Megalonyx & sp. & Mexico & 4,90 & 1,80 & SA-NA \\
\hline 20112 & J. Alroy & Xenarthra & Megalonychidae & Megalonyx & sp. & United States & 4,90 & 1,80 & SA-NA \\
\hline 20115 & J. Alroy & Xenarthra & Megalonychidae & Megalonyx & sp. & United States & 4,90 & 1,80 & SA-NA \\
\hline 20118 & J. Alroy & Xenarthra & Megalonychidae & Megalonyx & sp. & United States & 4,90 & 1,80 & SA-NA \\
\hline 20121 & J. Alroy & Xenarthra & Megalonychidae & Megalonyx & sp. & United States & 4,90 & 1,80 & SA-NA \\
\hline 20125 & J. Alroy & Xenarthra & Megalonychidae & Megalonyx & sp. & United States & 1,80 & 0,30 & SA-NA \\
\hline 20128 & J. Alroy & Xenarthra & Megalonychidae & Megalonyx & sp. & United States & 1,80 & 0,30 & SA-NA \\
\hline 20130 & J. Alroy & Xenarthra & Megalonychidae & Megalonyx & sp. & United States & 1,80 & 0,30 & SA-NA \\
\hline 20155 & J. Alroy & Xenarthra & Megalonychidae & Megalonyx & sp. & United States & 1,80 & 0,30 & SA-NA \\
\hline
\end{tabular}




\begin{tabular}{|c|c|c|c|c|c|c|c|c|c|}
\hline 20161 & J. Alroy & Xenarthra & Megalonychidae & Megalonyx & sp. & United States & 1,80 & 0,30 & SA-NA \\
\hline 20187 & J. Alroy & Xenarthra & Megalonychidae & Megalonyx & sp. & United States & 1,80 & 0,30 & SA-NA \\
\hline 20272 & J. Alroy & Xenarthra & Megalonychidae & Megalonyx & sp. & United States & 1,80 & 0,30 & SA-NA \\
\hline 20273 & J. Alroy & Xenarthra & Megalonychidae & Megalonyx & sp. & United States & 1,80 & 0,30 & SA-NA \\
\hline 20291 & J. Alroy & Xenarthra & Megalonychidae & Megalonyx & sp. & United States & 1,80 & 0,30 & SA-NA \\
\hline 20301 & J. Alroy & Xenarthra & Megalonychidae & Megalonyx & sp. & United States & 1,80 & 0,30 & SA-NA \\
\hline 20302 & J. Alroy & Xenarthra & Megalonychidae & Megalonyx & sp. & United States & 1,80 & 0,30 & SA-NA \\
\hline 20319 & J. Alroy & Xenarthra & Megalonychidae & Megalonyx & sp. & United States & 1,80 & 0,30 & SA-NA \\
\hline 20323 & J. Alroy & Xenarthra & Megalonychidae & Megalonyx & sp. & United States & 1,80 & 0,01 & SA-NA \\
\hline 20327 & J. Alroy & Xenarthra & Megalonychidae & Megalonyx & sp. & United States & 1,80 & 0,30 & SA-NA \\
\hline 20387 & J. Alroy & Xenarthra & Megalonychidae & Megalonyx & sp. & United States & 1,80 & 0,30 & SA-NA \\
\hline 20491 & J. Alroy & Xenarthra & Megalonychidae & Megalonyx & sp. & United States & 0,30 & 0,01 & SA-NA \\
\hline 20563 & J. Alroy & Xenarthra & Megalonychidae & Megalonyx & sp. & Canada & 1,80 & 0,30 & SA-NA \\
\hline 20573 & J. Alroy & Xenarthra & Megalonychidae & Megalonyx & sp. & United States & 0,13 & 0,01 & SA-NA \\
\hline 20585 & J. Alroy & Xenarthra & Megalonychidae & Megalonyx & sp. & United States & 0,13 & 0,01 & SA-NA \\
\hline 20609 & J. Alroy & Xenarthra & Megalonychidae & Megalonyx & sp. & United States & 0,13 & 0,01 & SA-NA \\
\hline 20615 & J. Alroy & Xenarthra & Megalonychidae & Megalonyx & sp. & Canada & 0,13 & 0,01 & SA-NA \\
\hline 20639 & J. Alroy & Xenarthra & Megalonychidae & Megalonyx & sp. & United States & 0,13 & 0,01 & SA-NA \\
\hline 26550 & J. Alroy & Xenarthra & Megalonychidae & Megalonyx & sp. & United States & 1,80 & 0,30 & SA-NA \\
\hline 34382 & M. Carrano & Xenarthra & Megalonychidae & Megalonyx & sp. & United States & 0,78 & 0,13 & SA-NA \\
\hline 65469 & M. Uhen & Xenarthra & Megalonychidae & Megalonyx & sp. & United States & 2,59 & 0,01 & SA-NA \\
\hline 70118 & J. Alroy & Xenarthra & Megalonychidae & Megalonyx & sp. & El Salvador & 2,59 & 0,13 & SA-NA \\
\hline 71312 & J. Alroy & Xenarthra & Megalonychidae & Megalonyx & sp. & Guatemala & 0,13 & 0,01 & SA-NA \\
\hline 73752 & M. Uhen & Xenarthra & Megalonychidae & Megalonyx & sp. & United States & 0,13 & 0,01 & SA-NA \\
\hline 74235 & J. Alroy & Xenarthra & Megalonychidae & Megalonyx & sp. & United States & 0,13 & 0,01 & SA-NA \\
\hline 74294 & J. Alroy & Xenarthra & Megalonychidae & Megalonyx & sp. & United States & 0,13 & 0,01 & SA-NA \\
\hline 84189 & M. Uhen & Xenarthra & Megalonychidae & Megalonyx & sp. & United States & 0,13 & 0,01 & SA-NA \\
\hline 93250 & J. Alroy & Xenarthra & Megalonychidae & Megalonyx & sp. & United States & 0,13 & 0,01 & SA-NA \\
\hline 93332 & J. Alroy & Xenarthra & Megalonychidae & Megalonyx & sp. & United States & 0,03 & 0,03 & SA-NA \\
\hline 20152 & J. Alroy & Xenarthra & Megalonychidae & Meizonyx & salvadorensis & El Salvador & 1,80 & 0,30 & SA-NA \\
\hline 19290 & J. Alroy & Xenarthra & Megalonychidae & Pliometanastes & galushai & United States & 10,30 & 4,90 & SA-NA \\
\hline 18072 & J. Alroy & Xenarthra & Megalonychidae & Pliometanastes & protistus & United States & 10,30 & 4,90 & SA-NA \\
\hline 18529 & J. Alroy & Xenarthra & Megalonychidae & Pliometanastes & protistus & United States & 10,30 & 4,90 & SA-NA \\
\hline 18560 & J. Alroy & Xenarthra & Megalonychidae & Pliometanastes & protistus & United States & 13,60 & 10,30 & SA-NA \\
\hline 18564 & J. Alroy & Xenarthra & Megalonychidae & Pliometanastes & protistus & United States & 10,30 & 4,90 & SA-NA \\
\hline 18601 & J. Alroy & Xenarthra & Megalonychidae & Pliometanastes & protistus & United States & 10,30 & 4,90 & SA-NA \\
\hline 19546 & J. Alroy & Xenarthra & Megalonychidae & Pliometanastes & protistus & United States & 10,30 & 4,90 & SA-NA \\
\hline Valerio and Laurito 2014 & M. Vallejo & Xenarthra & Megalonychidae & Pliometanastes & protistus & Costa Rica & 5,30 & 2,70 & SA-NA \\
\hline 18086 & J. Alroy & Xenarthra & Megalonychidae & Pliometanastes & sp. & United States & 10,30 & 4,90 & SA-NA \\
\hline 18539 & J. Alroy & Xenarthra & Megalonychidae & Pliometanastes & sp. & United States & 10,30 & 4,90 & SA-NA \\
\hline 19186 & J. Alroy & Xenarthra & Megalonychidae & Pliometanastes & sp. & United States & 10,30 & 4,90 & SA-NA \\
\hline 19411 & J. Alroy & Xenarthra & Megalonychidae & Pliometanastes & sp. & United States & 10,30 & 4,90 & SA-NA \\
\hline 19748 & J. Alroy & Xenarthra & Megatheriidae & Eremotherium & eomigrans & United States & 4,90 & 1,80 & SA-NA \\
\hline 19750 & J. Alroy & Xenarthra & Megatheriidae & Eremotherium & eomigrans & United States & 4,90 & 0,30 & SA-NA \\
\hline 20299 & J. Alroy & Xenarthra & Megatheriidae & Eremotherium & eomigrans & United States & 1,80 & 0,30 & SA-NA \\
\hline 20308 & J. Alroy & Xenarthra & Megatheriidae & Eremotherium & eomigrans & United States & 1,80 & 0,30 & SA-NA \\
\hline 20350 & J. Alroy & Xenarthra & Megatheriidae & Eremotherium & eomigrans & United States & 1,80 & 0,30 & SA-NA \\
\hline 20384 & J. Alroy & Xenarthra & Megatheriidae & Eremotherium & eomigrans & United States & 4,90 & 1,80 & SA-NA \\
\hline 20415 & J. Alroy & Xenarthra & Megatheriidae & Eremotherium & eomigrans & United States & 1,80 & 0,30 & SA-NA \\
\hline 20475 & J. Alroy & Xenarthra & Megatheriidae & Eremotherium & eomigrans & United States & 1,80 & 0,30 & SA-NA \\
\hline 98313 & M. Uhen & Xenarthra & Megatheriidae & Eremotherium & eomigrans & United States & 4,90 & 0,30 & SA-NA \\
\hline 20395 & J. Alroy & Xenarthra & Megatheriidae & Eremotherium & laurillardi & Panama & 1,80 & 0,30 & SA-NA \\
\hline 20591 & J. Alroy & Xenarthra & Megatheriidae & Eremotherium & laurillardi & United States & 0,13 & 0,01 & SA-NA \\
\hline 20604 & J. Alroy & Xenarthra & Megatheriidae & Eremotherium & laurillardi & United States & 0,13 & 0,01 & SA-NA \\
\hline 65405 & M. Uhen & Xenarthra & Megatheriidae & Eremotherium & laurillardi & United States & 2,59 & 0,01 & SA-NA \\
\hline 71267 & J. Alroy & Xenarthra & Megatheriidae & Eremotherium & laurillardi & Nicaragua & 0,13 & 0,01 & SA-NA \\
\hline 71278 & J. Alroy & Xenarthra & Megatheriidae & Eremotherium & laurillardi & Panama & 0,13 & 0,01 & SA-NA \\
\hline 79750 & M. Uhen & Xenarthra & Megatheriidae & Eremotherium & laurillardi & United States & 0,30 & 0,01 & SA-NA \\
\hline 79778 & M. Uhen & Xenarthra & Megatheriidae & Eremotherium & laurillardi & United States & 0,78 & 0,13 & SA-NA \\
\hline 86962 & M. Uhen & Xenarthra & Megatheriidae & Eremotherium & laurillardi & Nicaragua & 23,03 & 2,59 & SA-NA \\
\hline 96899 & M. Uhen & Xenarthra & Megatheriidae & Eremotherium & laurillardi & United States & 2,59 & 0,01 & SA-NA \\
\hline Lucas 2014 & M. Vallejo & Xenarthra & Megatheriidae & Eremotherium & laurillardi & Panama & 0,47 & 0,45 & SA-NA \\
\hline 19747 & J. Alroy & Xenarthra & Megatheriidae & Eremotherium & sp. & United States & 4,90 & 1,80 & SA-NA \\
\hline 19754 & J. Alroy & Xenarthra & Megatheriidae & Eremotherium & sp. & United States & 4,90 & 1,80 & SA-NA \\
\hline 19755 & J. Alroy & Xenarthra & Megatheriidae & Eremotherium & sp. & United States & 4,90 & 1,80 & SA-NA \\
\hline 20152 & J. Alroy & Xenarthra & Megatheriidae & Eremotherium & sp. & El Salvador & 1,80 & 0,30 & SA-NA \\
\hline 20351 & J. Alroy & Xenarthra & Megatheriidae & Eremotherium & sp. & United States & 1,80 & 0,30 & SA-NA \\
\hline 20394 & J. Alroy & Xenarthra & Megatheriidae & Eremotherium & sp. & United States & 1,80 & 0,30 & SA-NA \\
\hline 20416 & J. Alroy & Xenarthra & Megatheriidae & Eremotherium & sp. & United States & 1,80 & 0,30 & SA-NA \\
\hline 20478 & J. Alroy & Xenarthra & Megatheriidae & Eremotherium & sp. & United States & 1,80 & 0,30 & SA-NA \\
\hline 58089 & M. Uhen & Xenarthra & Megatheriidae & Eremotherium & sp. & United States & 1,80 & 0,30 & SA-NA \\
\hline 69920 & J. Alroy & Xenarthra & Megatheriidae & Eremotherium & sp. & Costa Rica & 3,60 & 0,78 & SA-NA \\
\hline 70118 & J. Alroy & Xenarthra & Megatheriidae & Eremotherium & sp. & El Salvador & 2,59 & 0,13 & SA-NA \\
\hline 71294 & J. Alroy & Xenarthra & Megatheriidae & Eremotherium & sp. & Guatemala & 0,13 & 0,01 & SA-NA \\
\hline 79759 & M. Uhen & Xenarthra & Megatheriidae & Eremotherium & sp. & United States & 1,80 & 0,30 & SA-NA \\
\hline 77596 & M. Uhen & Xenarthra & Megatheriidae & Megatherium & americanum & United States & 2,59 & 0,01 & SA-NA \\
\hline 96899 & M. Uhen & Xenarthra & Megatheriidae & Megatherium & americanum & United States & 2,59 & 0,01 & SA-NA \\
\hline 77596 & M. Uhen & Xenarthra & Megatheriidae & Megatherium & mirable & United States & 2,59 & 0,01 & SA-NA \\
\hline 71312 & J. Alroy & Xenarthra & Megatheriidae & Megatherium & sp. & Guatemala & 0,13 & 0,01 & SA-NA \\
\hline
\end{tabular}




\begin{tabular}{|c|c|c|c|c|c|c|c|c|c|}
\hline 86959 & M. Uhen & Xenarthra & Megatheriidae & Megatherium & sp. & Nicaragua & 2,59 & 0,01 & SA-NA \\
\hline 18738 & J. Alroy & Xenarthra & Mylodontidae & Glossotherium & sp. & Mexico & 10,30 & 4,90 & SA-NA \\
\hline 19658 & J. Alroy & Xenarthra & Mylodontidae & Glossotherium & chapadmalense & United States & 4,90 & 1,80 & SA-NA \\
\hline 19745 & J. Alroy & Xenarthra & Mylodontidae & Glossotherium & chapadmalense & United States & 4,90 & 1,80 & SA-NA \\
\hline 19752 & J. Alroy & Xenarthra & Mylodontidae & Glossotherium & chapadmalense & United States & 4,90 & 1,80 & SA-NA \\
\hline 19753 & J. Alroy & Xenarthra & Mylodontidae & Glossotherium & chapadmalense & United States & 4,90 & 1,80 & SA-NA \\
\hline 19754 & J. Alroy & Xenarthra & Mylodontidae & Glossotherium & chapadmalense & United States & 4,90 & 1,80 & SA-NA \\
\hline 19755 & J. Alroy & Xenarthra & Mylodontidae & Glossotherium & chapadmalense & United States & 4,90 & 1,80 & SA-NA \\
\hline 19758 & J. Alroy & Xenarthra & Mylodontidae & Glossotherium & chapadmalense & United States & 4,90 & 1,80 & SA-NA \\
\hline 19973 & J. Alroy & Xenarthra & Mylodontidae & Glossotherium & chapadmalense & United States & 4,90 & 1,80 & SA-NA \\
\hline 20023 & J. Alroy & Xenarthra & Mylodontidae & Glossotherium & chapadmalense & United States & 4,90 & 1,80 & SA-NA \\
\hline 20077 & J. Alroy & Xenarthra & Mylodontidae & Glossotherium & garbanii & Mexico & 4,90 & 1,80 & SA-NA \\
\hline 113275 & J. Alroy & Xenarthra & Mylodontidae & Glossotherium & garbanii & United States & 4,90 & 1,80 & SA-NA \\
\hline 71278 & J. Alroy & Xenarthra & Mylodontidae & Glossotherium & tropicorum & Panama & 0,13 & 0,01 & SA-NA \\
\hline 20636 & J. Alroy & Xenarthra & Mylodontidae & Glossotherium & sp. & United States & 0,13 & 0,01 & SA-NA \\
\hline 70113 & J. Alroy & Xenarthra & Mylodontidae & Glossotherium & sp. & Mexico & 4,90 & 1,80 & SA-NA \\
\hline 70117 & J. Alroy & Xenarthra & Mylodontidae & Glossotherium & sp. & Mexico & 4,90 & 1,80 & SA-NA \\
\hline 71312 & J. Alroy & Xenarthra & Mylodontidae & Mylodon & sp. & Guatemala & 0,13 & 0,01 & SA-NA \\
\hline 104046 & M. Uhen & Xenarthra & Mylodontidae & Mylodon & sp. & United States & 0,13 & 0,01 & SA-NA \\
\hline 19744 & J. Alroy & Xenarthra & Mylodontidae & Paramylodon & sp. & United States & 4,90 & 1,80 & SA-NA \\
\hline 19915 & J. Alroy & Xenarthra & Mylodontidae & Paramylodon & harlani & United States & 4,90 & 1,80 & SA-NA \\
\hline 19916 & J. Alroy & Xenarthra & Mylodontidae & Paramylodon & harlani & United States & 4,90 & 1,80 & SA-NA \\
\hline 20127 & J. Alroy & Xenarthra & Mylodontidae & Paramylodon & harlani & United States & 1,80 & 0,30 & SA-NA \\
\hline 20143 & J. Alroy & Xenarthra & Mylodontidae & Paramylodon & harlani & United States & 1,80 & 0,30 & SA-NA \\
\hline 20165 & J. Alroy & Xenarthra & Mylodontidae & Paramylodon & harlani & United States & 1,80 & 0,30 & SA-NA \\
\hline 20259 & J. Alroy & Xenarthra & Mylodontidae & Paramylodon & harlani & United States & 1,80 & 0,30 & SA-NA \\
\hline 20283 & J. Alroy & Xenarthra & Mylodontidae & Paramylodon & harlani & Mexico & 1,80 & 0,30 & SA-NA \\
\hline 20293 & J. Alroy & Xenarthra & Mylodontidae & Paramylodon & harlani & United States & 1,80 & 0,30 & SA-NA \\
\hline 20295 & J. Alroy & Xenarthra & Mylodontidae & Paramylodon & harlani & United States & 1,80 & 0,30 & SA-NA \\
\hline 20299 & J. Alroy & Xenarthra & Mylodontidae & Paramylodon & harlani & United States & 1,80 & 0,30 & SA-NA \\
\hline 20307 & J. Alroy & Xenarthra & Mylodontidae & Paramylodon & harlani & United States & 1,80 & 0,30 & SA-NA \\
\hline 20310 & J. Alroy & Xenarthra & Mylodontidae & Paramylodon & harlani & United States & 1,80 & 0,30 & SA-NA \\
\hline 20327 & J. Alroy & Xenarthra & Mylodontidae & Paramylodon & harlani & United States & 1,80 & 0,30 & SA-NA \\
\hline 20342 & J. Alroy & Xenarthra & Mylodontidae & Paramylodon & harlani & United States & 1,80 & 0,30 & SA-NA \\
\hline 20347 & J. Alroy & Xenarthra & Mylodontidae & Paramylodon & harlani & United States & 1,80 & 0,30 & SA-NA \\
\hline 20350 & J. Alroy & Xenarthra & Mylodontidae & Paramylodon & harlani & United States & 1,80 & 0,30 & SA-NA \\
\hline 20353 & J. Alroy & Xenarthra & Mylodontidae & Paramylodon & harlani & United States & 1,80 & 0,30 & SA-NA \\
\hline 20375 & J. Alroy & Xenarthra & Mylodontidae & Paramylodon & harlani & United States & 1,80 & 0,30 & SA-NA \\
\hline 20382 & J. Alroy & Xenarthra & Mylodontidae & Paramylodon & harlani & United States & 1,80 & 0,30 & SA-NA \\
\hline 20384 & J. Alroy & Xenarthra & Mylodontidae & Paramylodon & harlani & United States & 4,90 & 1,80 & SA-NA \\
\hline 20385 & J. Alroy & Xenarthra & Mylodontidae & Paramylodon & harlani & United States & 1,80 & 0,30 & SA-NA \\
\hline 20387 & J. Alroy & Xenarthra & Mylodontidae & Paramylodon & harlani & United States & $\begin{array}{l}1,80 \\
1,80\end{array}$ & 0,30 & SA-NA \\
\hline 20390 & J. Alroy & Xenarthra & Mylodontidae & Paramylodon & harlani & United States & 1,80 & 0,30 & SA-NA \\
\hline 20397 & J. Alroy & Xenarthra & Mylodontidae & Paramylodon & harlani & United States & 4,90 & 0,30 & SA-NA \\
\hline 20400 & J. Alroy & Xenarthra & Mylodontidae & Paramylodon & harlani & United States & 1,80 & 0,30 & SA-NA \\
\hline 20402 & J. Alroy & Xenarthra & Mylodontidae & Paramylodon & harlani & United States & 1,80 & 0,30 & SA-NA \\
\hline 20417 & J. Alroy & Xenarthra & Mylodontidae & Paramylodon & harlani & Canada & 1,80 & 0,30 & SA-NA \\
\hline 20443 & J. Alroy & Xenarthra & Mylodontidae & Paramylodon & harlani & United States & 1,80 & 0,30 & SA-NA \\
\hline 20454 & J. Alroy & Xenarthra & Mylodontidae & Paramylodon & harlani & United States & 1,80 & 0,30 & SA-NA \\
\hline 20455 & J. Alroy & Xenarthra & Mylodontidae & Paramylodon & harlani & United States & 1,80 & 0,30 & SA-NA \\
\hline 20456 & J. Alroy & Xenarthra & Mylodontidae & Paramylodon & harlani & United States & 1,80 & 0,30 & SA-NA \\
\hline 20475 & J. Alroy & Xenarthra & Mylodontidae & Paramylodon & harlani & United States & 1,80 & 0,30 & SA-NA \\
\hline 20479 & J. Alroy & Xenarthra & Mylodontidae & Paramylodon & harlani & United States & 1,80 & 0,30 & SA-NA \\
\hline 20484 & J. Alroy & Xenarthra & Mylodontidae & Paramylodon & harlani & United States & 1,80 & 0,30 & SA-NA \\
\hline 20487 & J. Alroy & Xenarthra & Mylodontidae & Paramylodon & harlani & United States & 1,80 & 0,01 & SA-NA \\
\hline 20491 & J. Alroy & Xenarthra & Mylodontidae & Paramylodon & harlani & United States & 0,30 & 0,01 & SA-NA \\
\hline 20498 & J. Alroy & Xenarthra & Mylodontidae & Paramylodon & harlani & United States & 1,80 & 0,30 & SA-NA \\
\hline 20499 & J. Alroy & Xenarthra & Mylodontidae & Paramylodon & harlani & United States & 1,80 & 0,30 & SA-NA \\
\hline 20520 & J. Alroy & Xenarthra & Mylodontidae & Paramylodon & harlani & United States & 1,80 & 0,30 & SA-NA \\
\hline 20556 & J. Alroy & Xenarthra & Mylodontidae & Paramylodon & harlani & United States & 1,80 & 0,30 & SA-NA \\
\hline 20565 & J. Alroy & Xenarthra & Mylodontidae & Paramylodon & harlani & United States & 1,80 & 0,30 & SA-NA \\
\hline 20569 & J. Alroy & Xenarthra & Mylodontidae & Paramylodon & harlani & United States & 0,13 & 0,01 & SA-NA \\
\hline 20591 & J. Alroy & Xenarthra & Mylodontidae & Paramylodon & harlani & United States & 0,13 & 0,01 & SA-NA \\
\hline 20604 & J. Alroy & Xenarthra & Mylodontidae & Paramylodon & harlani & United States & 0,13 & 0,01 & SA-NA \\
\hline 20605 & J. Alroy & Xenarthra & Mylodontidae & Paramylodon & harlani & United States & 0,13 & 0,01 & SA-NA \\
\hline 20612 & J. Alroy & Xenarthra & Mylodontidae & Paramylodon & harlani & United States & 0,13 & 0,01 & SA-NA \\
\hline 20623 & J. Alroy & Xenarthra & Mylodontidae & Paramylodon & harlani & United States & 0,13 & 0,01 & SA-NA \\
\hline 20630 & J. Alroy & Xenarthra & Mylodontidae & Paramylodon & harlani & United States & 0,30 & 0,01 & SA-NA \\
\hline 20641 & J. Alroy & Xenarthra & Mylodontidae & Paramylodon & harlani & United States & 0,13 & 0,01 & SA-NA \\
\hline 20642 & J. Alroy & Xenarthra & Mylodontidae & Paramylodon & harlani & United States & 0,13 & 0,01 & SA-NA \\
\hline 20647 & J. Alroy & Xenarthra & Mylodontidae & Paramylodon & harlani & United States & 0,13 & 0,01 & SA-NA \\
\hline 28225 & J. Alroy & Xenarthra & Mylodontidae & Paramylodon & harlani & United States & 1,80 & 0,30 & SA-NA \\
\hline 58089 & M. Uhen & Xenarthra & Mylodontidae & Paramylodon & harlani & United States & 1,80 & 0,30 & SA-NA \\
\hline 65405 & M. Uhen & Xenarthra & Mylodontidae & Paramylodon & harlani & United States & 2,59 & 0,01 & SA-NA \\
\hline 83072 & J. Alroy & Xenarthra & Mylodontidae & Paramylodon & harlani & United States & 0,13 & 0,01 & SA-NA \\
\hline 85144 & J. Alroy & Xenarthra & Mylodontidae & Paramylodon & harlani & United States & 0,13 & 0,01 & SA-NA \\
\hline 85145 & J. Alroy & Xenarthra & Mylodontidae & Paramylodon & harlani & United States & 2,59 & 0,78 & SA-NA \\
\hline 93137 & J. Alroy & Xenarthra & Mylodontidae & Paramylodon & harlani & United States & 2,59 & 0,01 & SA-NA \\
\hline 93143 & J. Alroy & Xenarthra & Mylodontidae & Paramylodon & harlani & United States & 0,02 & 0,01 & SA-NA \\
\hline
\end{tabular}




\begin{tabular}{|c|c|c|c|c|c|c|c|c|c|}
\hline 93251 & J. Alroy & Xenarthra & Mylodontidae & Paramylodon & harlani & United States & 0,13 & 0,01 & SA-NA \\
\hline 93483 & J. Alroy & Xenarthra & Mylodontidae & Paramylodon & harlani & United States & 0,13 & 0,01 & SA-NA \\
\hline 98313 & M. Uhen & Xenarthra & Mylodontidae & Paramylodon & harlani & United States & 4,90 & 0,30 & SA-NA \\
\hline Lucas 2014 & M. Vallejo & Xenarthra & Mylodontidae & Paramylodon & harlani & Panama & 0,47 & 0,45 & SA-NA \\
\hline 20033 & J. Alroy & Xenarthra & Mylodontidae & Paramylodon & sp. & United States & 4,90 & 1,80 & SA-NA \\
\hline 20050 & J. Alroy & Xenarthra & Mylodontidae & Paramylodon & sp. & United States & 4,90 & 1,80 & SA-NA \\
\hline 20051 & J. Alroy & Xenarthra & Mylodontidae & Paramylodon & sp. & United States & 4,90 & 1,80 & SA-NA \\
\hline 20154 & J. Alroy & Xenarthra & Mylodontidae & Paramylodon & sp. & United States & 1,80 & 0,30 & SA-NA \\
\hline 20261 & J. Alroy & Xenarthra & Mylodontidae & Paramylodon & sp. & United States & 1,80 & 0,30 & SA-NA \\
\hline 20279 & J. Alroy & Xenarthra & Mylodontidae & Paramylodon & sp. & United States & 1,80 & 0,30 & SA-NA \\
\hline 20322 & J. Alroy & Xenarthra & Mylodontidae & Paramylodon & sp. & Mexico & 4,90 & 1,80 & SA-NA \\
\hline 20468 & J. Alroy & Xenarthra & Mylodontidae & Paramylodon & sp. & United States & 1,80 & 0,30 & SA-NA \\
\hline 20495 & J. Alroy & Xenarthra & Mylodontidae & Paramylodon & sp. & Mexico & 0,30 & 0,01 & SA-NA \\
\hline 26550 & J. Alroy & Xenarthra & Mylodontidae & Paramylodon & sp. & United States & 1,80 & 0,30 & SA-NA \\
\hline 37788 & J. Head & Xenarthra & Mylodontidae & Paramylodon & sp. & United States & 0,13 & 0,01 & SA-NA \\
\hline 71312 & J. Alroy & Xenarthra & Mylodontidae & Paramylodon & sp. & Guatemala & 0,13 & 0,01 & SA-NA \\
\hline 74244 & J. Alroy & Xenarthra & Mylodontidae & Paramylodon & sp. & United States & 2,59 & 0,01 & SA-NA \\
\hline 93249 & J. Alroy & Xenarthra & Mylodontidae & Paramylodon & sp. & United States & 2,59 & 0,01 & SA-NA \\
\hline 71278 & J. Alroy & Xenarthra & Mylodontidae & Scelidotherium & sp. & Panama & 0,13 & 0,01 & SA-NA \\
\hline 18560 & J. Alroy & Xenarthra & Mylodontidae & Thinobadistes & segnis & United States & 13,60 & 10,30 & SA-NA \\
\hline 18563 & J. Alroy & Xenarthra & Mylodontidae & Thinobadistes & segnis & United States & 10,30 & 4,90 & SA-NA \\
\hline 18601 & J. Alroy & Xenarthra & Mylodontidae & Thinobadistes & wetzeli & United States & 10,30 & 4,90 & SA-NA \\
\hline 26549 & J. Alroy & Xenarthra & Mylodontidae & Thinobadistes & wetzeli & United States & 10,30 & 4,90 & SA-NA \\
\hline 18072 & J. Alroy & Xenarthra & Mylodontidae & Thinobadistes & wetzeli & United States & 10,30 & 4,90 & SA-NA \\
\hline 18086 & J. Alroy & Xenarthra & Mylodontidae & Thinobadistes & sp. & United States & 10,30 & 4,90 & SA-NA \\
\hline 18097 & J. Alroy & Xenarthra & Mylodontidae & Thinobadistes & sp. & United States & 10,30 & 4,90 & SA-NA \\
\hline 20322 & J. Alroy & Xenarthra & Myrmecophagidae & Myrmecophaga & tridactyla & Mexico & 4,90 & 1,80 & SA-NA \\
\hline 20117 & J. Alroy & Xenarthra & Nothrotheriidae & Nothrotheriops & shastensis & United States & 4,90 & 1,80 & SA-NA \\
\hline 20119 & J. Alroy & Xenarthra & Nothrotheriidae & Nothrotheriops & shastensis & United States & 4,90 & 1,80 & SA-NA \\
\hline 20120 & J. Alroy & Xenarthra & Nothrotheriidae & Nothrotheriops & shastensis & United States & 4,90 & 1,80 & SA-NA \\
\hline 20121 & J. Alroy & Xenarthra & Nothrotheriidae & Nothrotheriops & shastensis & United States & 4,90 & 1,80 & SA-NA \\
\hline 20123 & J. Alroy & Xenarthra & Nothrotheriidae & Nothrotheriops & shastensis & United States & 1,80 & 0,30 & SA-NA \\
\hline 20126 & J. Alroy & Xenarthra & Nothrotheriidae & Nothrotheriops & shastensis & United States & 1,80 & 0,30 & SA-NA \\
\hline 20273 & J. Alroy & Xenarthra & Nothrotheriidae & Nothrotheriops & shastensis & United States & 1,80 & 0,30 & SA-NA \\
\hline 20283 & J. Alroy & Xenarthra & Nothrotheriidae & Nothrotheriops & shastensis & Mexico & 1,80 & 0,30 & SA-NA \\
\hline 20327 & J. Alroy & Xenarthra & Nothrotheriidae & Nothrotheriops & shastensis & United States & 1,80 & 0,30 & SA-NA \\
\hline 20624 & J. Alroy & Xenarthra & Nothrotheriidae & Nothrotheriops & shastensis & United States & 0,30 & 0,01 & SA-NA \\
\hline 20639 & J. Alroy & Xenarthra & Nothrotheriidae & Nothrotheriops & shastensis & United States & 0,13 & 0,01 & SA-NA \\
\hline 76059 & J. Alroy & Xenarthra & Nothrotheriidae & Nothrotheriops & shastensis & United States & 0,03 & 0,03 & SA-NA \\
\hline 76060 & J. Alroy & Xenarthra & Nothrotheriidae & Nothrotheriops & shastensis & United States & 0,13 & 0,01 & SA-NA \\
\hline 85143 & J. Alroy & Xenarthra & Nothrotheriidae & Nothrotheriops & shastensis & United States & 0,13 & 0,01 & SA-NA \\
\hline 93244 & J. Alroy & Xenarthra & Nothrotheriidae & Nothrotheriops & shastensis & United States & 0,13 & 0,01 & SA-NA \\
\hline 93245 & J. Alroy & Xenarthra & Nothrotheriidae & Nothrotheriops & shastensis & United States & 0,13 & 0,01 & SA-NA \\
\hline 93257 & J. Alroy & Xenarthra & Nothrotheriidae & Nothrotheriops & shastensis & United States & 2,59 & 0,01 & SA-NA \\
\hline 93374 & J. Alroy & Xenarthra & Nothrotheriidae & Nothrotheriops & shastensis & United States & 0,13 & 0,00 & SA-NA \\
\hline DeIuliis et al 2014 & M. Vallejo & Xenarthra & Nothrotheriidae & Nothrotheriops & shastensis & Belize & 0,13 & 0,00 & SA-NA \\
\hline 20137 & J. Alroy & Xenarthra & Nothrotheriidae & Nothrotheriops & texanus & United States & 1,80 & 0,30 & SA-NA \\
\hline 20322 & J. Alroy & Xenarthra & Nothrotheriidae & Nothrotheriops & texanus & Mexico & 4,90 & 1,80 & SA-NA \\
\hline 20342 & J. Alroy & Xenarthra & Nothrotheriidae & Nothrotheriops & texanus & United States & 1,80 & 0,30 & SA-NA \\
\hline 20387 & J. Alroy & Xenarthra & Nothrotheriidae & Nothrotheriops & texanus & United States & 1,80 & 0,30 & SA-NA \\
\hline 20400 & J. Alroy & Xenarthra & Nothrotheriidae & Nothrotheriops & texanus & United States & 1,80 & 0,30 & SA-NA \\
\hline 20478 & J. Alroy & Xenarthra & Nothrotheriidae & Nothrotheriops & texanus & United States & 1,80 & 0,30 & SA-NA \\
\hline 20484 & J. Alroy & Xenarthra & Nothrotheriidae & Nothrotheriops & texanus & United States & 1,80 & 0,30 & SA-NA \\
\hline 20161 & J. Alroy & Xenarthra & Nothrotheriidae & Nothrotheriops & sp. & United States & 1,80 & 0,30 & SA-NA \\
\hline 20171 & J. Alroy & Xenarthra & Nothrotheriidae & Nothrotheriops & sp. & United States & 1,80 & 0,30 & SA-NA \\
\hline 20272 & J. Alroy & Xenarthra & Nothrotheriidae & Nothrotheriops & sp. & United States & 1,80 & 0,30 & SA-NA \\
\hline 20285 & J. Alroy & Xenarthra & Nothrotheriidae & Nothrotheriops & sp. & Mexico & 1,80 & 0,30 & SA-NA \\
\hline 20294 & J. Alroy & Xenarthra & Nothrotheriidae & Nothrotheriops & sp. & United States & 1,80 & 0,30 & SA-NA \\
\hline 20335 & J. Alroy & Xenarthra & Nothrotheriidae & Nothrotheriops & sp. & United States & 1,80 & 0,01 & SA-NA \\
\hline 20578 & J. Alroy & Xenarthra & Nothrotheriidae & Nothrotheriops & sp. & United States & 0,13 & 0,01 & SA-NA \\
\hline 28224 & J. Alroy & Xenarthra & Nothrotheriidae & Nothrotheriops & sp. & United States & 1,80 & 0,30 & SA-NA \\
\hline 71345 & J. Alroy & Xenarthra & Nothrotheriidae & Nothrotherium & sp. & Mexico & 0,13 & 0,01 & SA-NA \\
\hline 19746 & J. Alroy & Xenarthra & Pampatheriidae & Holmesina & floridanus & United States & 4,90 & 1,80 & SA-NA \\
\hline 19749 & J. Alroy & Xenarthra & Pampatheriidae & Holmesina & floridanus & United States & 4,90 & 1,80 & SA-NA \\
\hline 19750 & J. Alroy & Xenarthra & Pampatheriidae & Holmesina & floridanus & United States & 4,90 & 0,30 & SA-NA \\
\hline 19751 & J. Alroy & Xenarthra & Pampatheriidae & Holmesina & floridanus & United States & 4,90 & 1,80 & SA-NA \\
\hline 19752 & J. Alroy & Xenarthra & Pampatheriidae & Holmesina & floridanus & United States & 4,90 & 1,80 & SA-NA \\
\hline 19753 & J. Alroy & Xenarthra & Pampatheriidae & Holmesina & floridanus & United States & 4,90 & 1,80 & SA-NA \\
\hline 19754 & J. Alroy & Xenarthra & Pampatheriidae & Holmesina & floridanus & United States & 4,90 & 1,80 & SA-NA \\
\hline 19758 & J. Alroy & Xenarthra & Pampatheriidae & Holmesina & floridanus & United States & 4,90 & 1,80 & SA-NA \\
\hline 19759 & J. Alroy & Xenarthra & Pampatheriidae & Holmesina & floridanus & United States & 4,90 & 1,80 & SA-NA \\
\hline 19764 & J. Alroy & Xenarthra & Pampatheriidae & Holmesina & floridanus & United States & 4,90 & 1,80 & SA-NA \\
\hline 20299 & J. Alroy & Xenarthra & Pampatheriidae & Holmesina & floridanus & United States & 1,80 & 0,30 & SA-NA \\
\hline 20308 & J. Alroy & Xenarthra & Pampatheriidae & Holmesina & floridanus & United States & 1,80 & 0,30 & SA-NA \\
\hline 20334 & J. Alroy & Xenarthra & Pampatheriidae & Holmesina & floridanus & United States & 1,80 & 0,30 & SA-NA \\
\hline 20350 & J. Alroy & Xenarthra & Pampatheriidae & Holmesina & floridanus & United States & 1,80 & 0,30 & SA-NA \\
\hline 20384 & J. Alroy & Xenarthra & Pampatheriidae & Holmesina & floridanus & United States & 4,90 & 1,80 & SA-NA \\
\hline 20385 & J. Alroy & Xenarthra & Pampatheriidae & Holmesina & floridanus & United States & 1,80 & 0,30 & SA-NA \\
\hline 20400 & J. Alroy & Xenarthra & Pampatheriidae & Holmesina & floridanus & United States & 1,80 & 0,30 & SA-NA \\
\hline
\end{tabular}




\begin{tabular}{|c|c|c|c|c|c|c|c|c|c|}
\hline 20475 & J. Alroy & Xenarthra & Pampatheriidae & Holmesina & floridanus & United States & 1,80 & 0,30 & SA-NA \\
\hline 20478 & J. Alroy & Xenarthra & Pampatheriidae & Holmesina & floridanus & United States & 1,80 & 0,30 & SA-NA \\
\hline 20482 & J. Alroy & Xenarthra & Pampatheriidae & Holmesina & floridanus & United States & 1,80 & 0,30 & SA-NA \\
\hline 20494 & J. Alroy & Xenarthra & Pampatheriidae & Holmesina & floridanus & United States & 1,80 & 0,30 & SA-NA \\
\hline 58089 & M. Uhen & Xenarthra & Pampatheriidae & Holmesina & floridanus & United States & 1,80 & 0,30 & SA-NA \\
\hline 98313 & M. Uhen & Xenarthra & Pampatheriidae & Holmesina & floridanus & United States & 4,90 & 0,30 & SA-NA \\
\hline 98320 & M. Uhen & Xenarthra & Pampatheriidae & Holmesina & floridanus & United States & 4,90 & 1,80 & SA-NA \\
\hline 113275 & J. Alroy & Xenarthra & Pampatheriidae & Holmesina & floridanus & United States & 4,90 & 1,80 & SA-NA \\
\hline 20283 & J. Alroy & Xenarthra & Pampatheriidae & Holmesina & septentrionalis & Mexico & 1,80 & 0,30 & SA-NA \\
\hline 20285 & J. Alroy & Xenarthra & Pampatheriidae & Holmesina & septentrionalis & Mexico & 1,80 & 0,30 & SA-NA \\
\hline 20289 & J. Alroy & Xenarthra & Pampatheriidae & Holmesina & septentrionalis & United States & 1,80 & 0,30 & SA-NA \\
\hline 20342 & J. Alroy & Xenarthra & Pampatheriidae & Holmesina & septentrionalis & United States & 1,80 & 0,30 & SA-NA \\
\hline 20390 & J. Alroy & Xenarthra & Pampatheriidae & Holmesina & septentrionalis & United States & 1,80 & 0,30 & SA-NA \\
\hline 20415 & J. Alroy & Xenarthra & Pampatheriidae & Holmesina & septentrionalis & United States & 1,80 & 0,30 & SA-NA \\
\hline 20440 & J. Alroy & Xenarthra & Pampatheriidae & Holmesina & septentrionalis & United States & 0,30 & 0,01 & SA-NA \\
\hline 20491 & J. Alroy & Xenarthra & Pampatheriidae & Holmesina & septentrionalis & United States & 0,30 & 0,01 & SA-NA \\
\hline 20520 & J. Alroy & Xenarthra & Pampatheriidae & Holmesina & septentrionalis & United States & 1,80 & 0,30 & SA-NA \\
\hline 20530 & J. Alroy & Xenarthra & Pampatheriidae & Holmesina & septentrionalis & United States & 1,80 & 0,30 & SA-NA \\
\hline 20571 & J. Alroy & Xenarthra & Pampatheriidae & Holmesina & septentrionalis & United States & 0,13 & 0,01 & SA-NA \\
\hline 20581 & J. Alroy & Xenarthra & Pampatheriidae & Holmesina & septentrionalis & United States & 0,13 & 0,01 & SA-NA \\
\hline 20586 & J. Alroy & Xenarthra & Pampatheriidae & Holmesina & septentrionalis & United States & 0,13 & 0,01 & SA-NA \\
\hline 20594 & J. Alroy & Xenarthra & Pampatheriidae & Holmesina & septentrionalis & United States & 0,13 & 0,01 & SA-NA \\
\hline 20603 & J. Alroy & Xenarthra & Pampatheriidae & Holmesina & septentrionalis & United States & 0,13 & 0,01 & SA-NA \\
\hline 20608 & J. Alroy & Xenarthra & Pampatheriidae & Holmesina & septentrionalis & United States & 0,13 & 0,01 & SA-NA \\
\hline 20613 & J. Alroy & Xenarthra & Pampatheriidae & Holmesina & septentrionalis & United States & 0,13 & 0,01 & SA-NA \\
\hline 20629 & J. Alroy & Xenarthra & Pampatheriidae & Holmesina & septentrionalis & United States & 0,13 & 0,01 & SA-NA \\
\hline 20634 & J. Alroy & Xenarthra & Pampatheriidae & Holmesina & septentrionalis & United States & 0,13 & 0,01 & SA-NA \\
\hline 20636 & J. Alroy & Xenarthra & Pampatheriidae & Holmesina & septentrionalis & United States & 0,13 & 0,01 & SA-NA \\
\hline 20638 & J. Alroy & Xenarthra & Pampatheriidae & Holmesina & septentrionalis & United States & 0,13 & 0,01 & SA-NA \\
\hline 20647 & J. Alroy & Xenarthra & Pampatheriidae & Holmesina & septentrionalis & United States & 0,13 & 0,01 & SA-NA \\
\hline 20653 & J. Alroy & Xenarthra & Pampatheriidae & Holmesina & septentrionalis & United States & 0,13 & 0,01 & SA-NA \\
\hline 34269 & M. Carrano & Xenarthra & Pampatheriidae & Holmesina & septentrionalis & United States & 0,13 & 0,01 & SA-NA \\
\hline 65405 & M. Uhen & Xenarthra & Pampatheriidae & Holmesina & septentrionalis & United States & 2,59 & 0,01 & SA-NA \\
\hline 79750 & M. Uhen & Xenarthra & Pampatheriidae & Holmesina & septentrionalis & United States & 0,30 & 0,01 & SA-NA \\
\hline 79757 & M. Uhen & Xenarthra & Pampatheriidae & Holmesina & septentrionalis & United States & 0,30 & 0,01 & SA-NA \\
\hline 79763 & M. Uhen & Xenarthra & Pampatheriidae & Holmesina & septentrionalis & United States & 0,13 & 0,01 & SA-NA \\
\hline 85136 & J. Alroy & Xenarthra & Pampatheriidae & Holmesina & septentrionalis & United States & 0,13 & 0,01 & SA-NA \\
\hline 85137 & J. Alroy & Xenarthra & Pampatheriidae & Holmesina & septentrionalis & United States & 2,59 & 0,01 & SA-NA \\
\hline 85138 & J. Alroy & Xenarthra & Pampatheriidae & Holmesina & septentrionalis & United States & 2,59 & 0,01 & SA-NA \\
\hline 85139 & J. Alroy & Xenarthra & Pampatheriidae & Holmesina & septentrionalis & United States & 2,59 & 0,01 & SA-NA \\
\hline 85140 & J. Alroy & Xenarthra & Pampatheriidae & Holmesina & septentrionalis & United States & 2,59 & 0,01 & SA-NA \\
\hline 85141 & J. Alroy & Xenarthra & Pampatheriidae & Holmesina & septentrionalis & United States & 2,59 & 0,01 & SA-NA \\
\hline 85142 & J. Alroy & Xenarthra & Pampatheriidae & Holmesina & septentrionalis & United States & 2,59 & 0,01 & SA-NA \\
\hline 93103 & J. Alroy & Xenarthra & Pampatheriidae & Holmesina & septentrionalis & United States & 0,13 & 0,01 & SA-NA \\
\hline 20285 & J. Alroy & Xenarthra & Pampatheriidae & Holmesina & sp. & Mexico & 1,80 & 0,30 & SA-NA \\
\hline 26550 & J. Alroy & Xenarthra & Pampatheriidae & Holmesina & sp. & United States & 1,80 & 0,30 & SA-NA \\
\hline 69920 & J. Alroy & Xenarthra & Pampatheriidae & Holmesina & sp. & Costa Rica & 3,60 & 0,78 & SA-NA \\
\hline 20495 & J. Alroy & Xenarthra & Pampatheriidae & Pampatherium & mexicanum & Mexico & 0,30 & 0,01 & SA-NA \\
\hline 85161 & J. Alroy & Xenarthra & Pampatheriidae & Pampatherium & mexicanum & Mexico & 2,59 & 0,01 & SA-NA \\
\hline 85162 & J. Alroy & Xenarthra & Pampatheriidae & Pampatherium & mexicanum & Mexico & 0,13 & 0,01 & SA-NA \\
\hline 69920 & J. Alroy & Xenarthra & Pampatheriidae & Pampatherium & sp. & Costa Rica & 3,60 & 0,78 & SA-NA \\
\hline 71312 & J. Alroy & Xenarthra & Pampatheriidae & Pampatherium & sp. & Guatemala & 0,13 & 0,01 & SA-NA \\
\hline Laurito and Valeiro 2013 & M. Vallejo & Xenarthra & Pampatheriidae & Scirrotherium & antelucanus & Costa Rica & 5,30 & 2,70 & SA-NA \\
\hline 70114 & J. Alroy & Xenarthra & Pampatheriidae & Vassallia & sp. & Mexico & 4,90 & 1,80 & SA-NA \\
\hline
\end{tabular}

PBDB is an abbreviation for the Paleobiology Database (fossilworks.org).

Literature cited

Castro, M.C., F.C. Montefeltro, and M.C. Langer (2014), The Quaternary vertebrate fauna of the limestone cave Gruta do Ioiô, northeastern Brazil, Quaternary International, v. 352, 164-175. De Iuliis, G., H.G. Mcdonald, N. Stanchly, J. Spenard, and G. Powis (2014), Nothrotheriops shastensis (Sinclair) from Actun Lak : First Record of Nothrotheriidae (Mammalia, Xenarthra, Pilosa) from Belize, Ameghiniana, 52, 153-171.

De Oliveira, P.V., A.M. Ribeiro, C.L. Ximenes, M.S.S. Viana, and E.C. Holanda (2014), Tayassuidae, Cervidae e Tapiridae da Gruta do Urso Fóssil, Holoceno, Parque Nacional de Ubajara, Ceará, Brasil, Revista Brasileira De Paleontologia, 17, 417-434.

Forasiepi, A.M., L.H. Soibelzon, C. Suarez Gomez, R. Sánchez, L.I. Quiroz, C. Jaramillo, and M.R. Sánchez-Villagra (2014), Carnivorans at the Great American Biotic Interchange: new discoveries from the northern neotropics, Naturwissenschaften, 101, 965-974.

Gonzalez, E., R. Labarca, M. Chavez-Hoffmeister, and M. Pino (2014), First Fossil Record of the Smallest Deer cf. Pudu Molina, 1782 (Artiodactyla , Cervidae), in the Late Pleistocene of South America, Journal of Vertebrate Paleontology, 34, 483-488.

Kerber, L., V.G. Pitana, A.M. Ribeiro, A.S. Hsiou, and E. V. Oliveira (2014), Late Pleistocene vertebrates from Touro Passo Creek ( Touro Passo Formation ), southern Brazil : a review, Revista Mexicana de Ciencias Geológicas, 31, 248-259.

Labarca, R., O.P. Recabarren, P. Canales-Brellenthin, and M. Pino (2014), The gomphotheres (proboscidea: Gomphotheriidae) from Pilauco site: Scavenging evidence in the Late Pleistocene of the Chilean Patagonia, Ouaternary International, 352, 75-84.

Laurito, C.A., and A.L. Valerio (2013), Scirrotherium antelucanus, una nueva especie de Pampatheriidae (Mammalia, Xenarthra, Cingulata) del Mioceno superior de Costa Rica, América Central, Revista Geologica de America Central, 49, 45-62.

Lindsey, E.L., and E.X. Lopez R (2015), Tanque Loma, a new late-Pleistocene megafaunal tar seep locality from southwest Ecuador, Journal of South American Earth Sciences, 57, 61-82. Lucas, S.G. (2014), Late Pleistocene mammals from El Hatillo, Panama, Revista Geologica de America Central, 50, $139-151$.

Luna, C.A., and L.E. Cruz (2014), Los mamíferos fósiles del Pleistoceno tardío-Holoceno temprano del sudeste de la provincia de Córdoba y sus implicancias paleoambientales para el centro de Argentina, Revista Brasileira de Paleontologia, 17, 69-82.

Pérez, E.A. (2013), Los Mamíferos Fósiles del Distrito De Puente de Piedra (Xenarthra, Glyptodontidae; Artiodactyla, Camelidae, Lamini), Grecia, Provincia de Alajuela, Costa Rica, Revista Geológica de América Central, 49, 33-44.

Pino, M., M. Chávez-Hoffmeister, X. Navarro-Harris, and R. Labarca (2013), The late Pleistocene Pilauco site, Osorno, south-central Chile, Quaternary International,299, 3-12. 
Ramirez, M.A., and F.J. Prevosti (2014), Systematic revision of "Canis" ensenadensis Ameghino, 1888 (Carnivora, Canidae) and the description of a new specimen from the pleistocene of Argentina, Ameghiniana, 51, 37-51.

Salles, L.O., J. Arroyo-Cabrales, A.C. Do, M. Lima, W. Lanzelotti, F.A. Perini, P.M. Velazco, and N.B. Simmons (2014), Quaternary Bats from the Impossível-Ioiô Cave System (Chapada Diamantina, Brazil), Humeral Remains and the First Fossil Record of Noctilio leporinus (Chiroptera, Noctilionidae) from South America, American Museum Novitates, 3798, 1-31.

Sertich, J.J.W., R.K. Stucky, H.G. McDonald, C. Newton, D.C. Fisher, E. Scott, Demboski J.R., C. Lucking, B.K. McHorse, and E.B. Davis (2014), High-elevation late Pleistocene (MIS 6-5) vertebrate faunas from the Ziegler Reservoir fossil site, Snowmass Village, Colorado, Quaternary Research, 82, 504-517.

Tovar, R.E., S. Sedov, M. Montellano-Ballesteros, and E. Solleiro (2014), The alluvial sequence of Santa Cruz Nuevo, Mexico: Paleoecological interpretation of fossil vertebrates and pedosediments from marine isotope stage 3, Revista Mexicana de Ciencias Geológicas, 31, 407-419.

Valerio, A.L., and C.A. Laurito (2014), Nueva evidencia de un estadio juvenil de Pliometanastes protistus y su relación con?Pliometanastes galushai Hirschfeld \& Webb, 1968 (Xernarthra, Megalonychidae) para el Mioceno Superior de Costa Rica, Revista Geológica de América Central, 51, 159-163. 


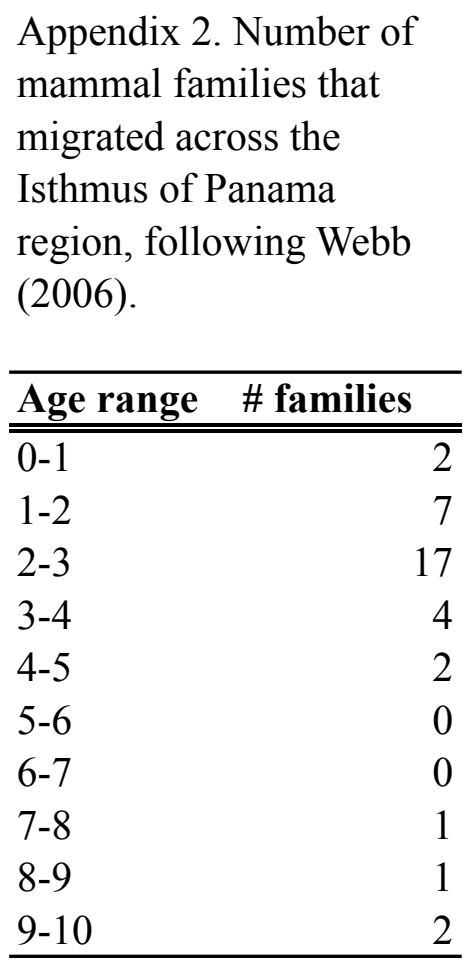


Appendix 3. Summary table of calculations to estimate the proportion of immigrants within 1 million year time intervals.

\begin{tabular}{cccc}
\hline Time slice & Occurrences & Number of different localities & Occurrences standardized by \# of total occurrences (Proportion) \\
\hline $0-1$ & 687 & 268 & 0,487 \\
$1-2$ & 222 & 114 & 0,157 \\
$2-3$ & 271 & 102 & 0,192 \\
$3-4$ & 11 & 6 & 0,008 \\
$4-5$ & 162 & 80 & 0,115 \\
$5-6$ & 8 & 5 & 0,006 \\
$6-7$ & 3 & 2 & 0,002 \\
$8-9$ & 6 & 32 & 0,004 \\
$10-11$ & 35 & 1 & 0,025 \\
$11-12$ & 2 & 1 & 0,001 \\
$13-14$ & 2 & 1 & 0,001 \\
$23-24$ & 1 & & 0,001 \\
\hline
\end{tabular}


Appendix 4. Compiliation of dated molecular phylogenies of American mammal clades that include at least one migrant population or species.

\begin{tabular}{|c|c|c|c|c|c|c|}
\hline & Class : Order : Family & Taxon / clade (constituent spp.) & Crown age & Lower & Upper & Reference \\
\hline 1 & Mammalia : Artiodactyla : Cervidae & South American species & $2.75^{*}$ & 2.5 & 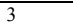 & Barbanti Duarte et al. 2008 \\
\hline 2 & Mammalia : Carnivora : Canidae & South American endemic clade & 4.3 & 3.4 & 5.5 & Perini et al. 2009 \\
\hline 3 & Mammalia : Carnivora : Felidae & Leopardus tigrinus & 3.7 & N/A & $\mathrm{N} / \mathrm{A}$ & Johnson et al. 1999 \\
\hline 4 & Mammalia : Carnivora : Mustelidae & Lontra longicaudis + L. felina vs. L. canadensis (node 14) & $3.1 *$ & 2.8 & 3.4 & Koepfli et al. 2008 \\
\hline 5 & Mammalia : Carnivora : Mustelidae & Pteronura brasiliensis vs. clade at Node 8 (node 7$)$ & 7.6 & 6.2 & 9 & Koepfli et al. 2008 \\
\hline 6 & Mammalia : Carnivora : Procyonidae & Nasua narica vs. N. nasua & $7.5^{*}$ & 7 & 8 & Koepfli et al. 2007 \\
\hline 7 & Mammalia : Carnivora : Procyonidae & Procyon cancrivorus vs. P. lotor & $5.35^{*}$ & 5 & 5.7 & Koepfli et al. 2007 \\
\hline 8 & Mammalia : Carnivora : Ursidae & Tremarctos ornatus vs. Arctodus simus & 5.66 & 4.26 & 7.34 & Krause et al. 2008 \\
\hline 9 & Mammalia : Chiroptera : Emballonuroidea & Centronycteris centralis & 5.5 & N/A & $\mathrm{N} / \mathrm{A}$ & $\operatorname{Lim} 2008$ \\
\hline 10 & Mammalia : Chiroptera : Emballonuroidea & Cormura brevirostris & 19.4 & $\mathrm{~N} / \mathrm{A}$ & $\mathrm{N} / \mathrm{A}$ & $\operatorname{Lim} 2008$ \\
\hline 11 & Mammalia : Chiroptera : Emballonuroidea & Cyttarops alecto & 14.6 & $\mathrm{~N} / \mathrm{A}$ & $\mathrm{N} / \mathrm{A}$ & Lim 2008 \\
\hline 12 & Mammalia : Chiroptera : Emballonuroidea & Diclidurus albus & 2 & N/A & $\mathrm{N} / \mathrm{A}$ & $\operatorname{Lim} 2008$ \\
\hline 13 & Mammalia : Chiroptera : Emballonuroidea & Peropteryx kappleri & 1 & $\mathrm{~N} / \mathrm{A}$ & N/A & $\operatorname{Lim} 2008$ \\
\hline 14 & Mammalia : Chiroptera : Emballonuroidea & Rhynchonycteris naso & 18 & N/A & N/A & $\operatorname{Lim} 2008$ \\
\hline 15 & Mammalia : Chiroptera : Emballonuroidea & Saccopteryx bilineata + S. leptura & 5.1 & $\mathrm{~N} / \mathrm{A}$ & $\mathrm{N} / \mathrm{A}$ & $\operatorname{Lim} 2008$ \\
\hline 16 & Mammalia : Chiroptera : Noctilionidae & Noctilio leporinus South American clade & 0.176 & N/A & $\mathrm{N} / \mathrm{A}$ & Pavan et al. 2013 \\
\hline 17 & Mammalia : Chiroptera : Phyllostomidae & Desmodus rotundus (CA x AMC, coalescent) & $0.95^{*}$ & 0.6 & 1.3 & Martins et al. 2009 \\
\hline 18 & Mammalia : Chiroptera : Vespertilionidae & Clade of Myotis levis, M. nigricans, $M$. oxyotus, M. albescens, M. chiloensis & 5 & N/A & N/A & Stadelmann et al. 2007 \\
\hline 19 & Mammalia : Chiroptera : Vespertilionidae & Clade of Myotis atacamensis, M. dominicensis, M. martiniquensis & 4 & N/A & $\mathrm{N} / \mathrm{A}$ & Stadelmann et al. 2007 \\
\hline 20 & Mammalia : Chiroptera : Vespertilionidae & Clade of Myotis elegans, M. riparius, M. simus, M. ruber, M. keaysi & 5.5 & N/A & $\mathrm{N} / \mathrm{A}$ & Stadelmann et al. 2007 \\
\hline 21 & Mammalia : Didelphimorpha : Didelphidae & Marmosa mexicanum & 3.5 & N/A & $\mathrm{N} / \mathrm{A}$ & Jansa et al. 2014 \\
\hline 22 & Mammalia : Didelphimorpha : Didelphidae & Didelphis virginiana & 3 & N/A & $\mathrm{N} / \mathrm{A}$ & Jansa et al. 2014 \\
\hline 23 & Mammalia : Didelphimorpha : Didelphidae & Tlacuatzin canescens & 17 & N/A & N/A & Jansa et al. 2014 \\
\hline 24 & Mammalia : Primates : Atelidae & Alouatta pigra + A. palliata & 4 & N/A & $\mathrm{N} / \mathrm{A}$ & Aristide et al. 2015 \\
\hline 25 & Mammalia : Primates : Atelidae & Ateles geoffroyi + A. fusciceps & 2.2 & 1.2 & 3.5 & Morales-Jimenez et al. 2015 \\
\hline 26 & Mammalia : Primates : Cebidae & Coastal + Andes Venezuelan vs. Colombian Andes + Central American Cebus & 1.86 & 1.13 & 2.67 & Lynch Alfaro et al. 2012 \\
\hline 27 & Mammalia : Primates : Cebidae & Saimiri oerstedii vs. S. sciureus & 0.95 & 0.47 & 1.48 & Lynch Alfaro et al. 2015 \\
\hline 28 & Mammalia : Rodentia : Cricetidae & Oryzomyalia crown & 8.2 & 7.3 & 9.2 & Leite et al. 2014 \\
\hline 29 & Mammalia : Rodentia : Cricetidae & Transandinomys talamancae & 3 & N/A & $\mathrm{N} / \mathrm{A}$ & Leite et al. 2014 \\
\hline 30 & Mammalia : Rodentia : Cricetidae & Oecomys trinitatis & 4 & N/A & $\mathrm{N} / \mathrm{A}$ & Leite et al. 2014 \\
\hline 31 & Mammalia : Rodentia : Cricetidae & Oligoryzomys fulvescens $+O$. nigripes & 1.75 & N/A & $\mathrm{N} / \mathrm{A}$ & Leite et al. 2014 \\
\hline 32 & Mammalia : Rodentia : Cricetidae & Nectomys squamipes & 3.5 & N/A & $\mathrm{N} / \mathrm{A}$ & Leite et al. 2014 \\
\hline 33 & Mammalia : Rodentia : Sciuridae & Sciurus South American Clade & 2.8 & 1.1 & 3.1 & Mercer and Roth 2003 \\
\hline
\end{tabular}

\section{Literature cited}

Aristide, L., A. L. Rosenberger, M. F. Tejedor, and S. I. Perez (2015), Modeling lineage and phenotypic diversification in the New World monkey (Platyrrhini, Primates) radiation, Molecular Phylogenetics and Evolution, 82, 375-385.

Barbanti Duarte, J. M., S. Gonzalez, and J. E. Maldonado (2008), The surprising history of South American deer. Molecular Phylogenetics and Evolution, 49, $17-22$.

Jansa, S. A., F. K. Barker, R. S. Voss (2014), The early diversification history of Didelphid marsupials: a window into South America's "Splendid Isolation", Evolution, 68, 684-695.

Johnson, W. E., J. P. Slattery, E. Eiziarik, J.-H. Kim, M. M. Raymond, C. Bonacic, R. Cambre, P. crawshaw, A. Nunes, H. N. Seuanez, M. A. Martins Moreira, K. L. Seymour, F. Simon, W. Swanson, and S. J. O'Brien (1999), Disparate phylogeographic patterns of molecular genetic variation in four closely related South American small cat species, Moleular Ecology, 8, S79-S94.

Koepfli, K.-P., K. A. Deere, G. J. Slater, C. Begg, K. Begg, L. Grassman, M. Lucherini, G. Veron, and R. K. Wayne (2008), Multigene phylogeny of the Mustelidae: Resolving relationships, tempo and biogeographic history of a mammalian adaptive radiation, BMC Biology, 6 , e10.

Koepfli, K.-P., M. E. Gompper, E. Eizirik, C.-C. Ho, L. Linden, J. E. Maldonado, and R. K. Wayne (2007), Phylogeny of the Procyonidae (Mammalia: Carnivora): Molecules, morphology and the Great American Interchange, Molecular Phylogenetics and Evolution, 43, 1076-1095.

Leite, R. N., S._O. Kolokotronis, F. C. Almeida, F. P. Werneck, D. S. Rogers, and M. Weksler (2014), In the wake of invasion: Tracing the historical biogeography of the South American Cricetid radiation (Rodentia, Sigmodontinae), PLoS ONE, 9, e100687.

Lim, B. K. (2008), Historical biogeography of New World emballonurid bats (tribe Diclidurini): taxon pulse diversification, Journal of Biogeography, 25, 1385-1401.

Lynch Alfaro, J. W., J. P. Boubli, L. E. Olson, A. Di Fiore, B. Wilson, G. A. Gutierrez-Espeleta, K. L. Chiou, M. Schulte, S. Neitzel, V. Ross, D. Schwochow, M. T. T. Nguyen, I. Farias, C. H. Janson, and M. E. Alfaro (2012), Explosive Pleistocene range expansion leads to widespread Amazonian sympatry between robust and gracile capuchin monkeys, Journal of Biogeography, $39,272-288$.

Lynch Alfaro, J. W., J. P. Boubli, F. P. Paim, C. C. Ribas, M. N. F. da Silva, M. R. Messias, F., Rohe, M. P. Merces, J. S. Silva Junior, C. R. Silva, G. M. Pinho, G. Koshkarian, M. T. T. Nguyen, M. L. Harada, R. M. Rabelo, H. L. Queiroz, M. E Alfaro, and I. P. Farias (2015), Biogeography of squirrel monkeys (genus Saimiri): South-central Amazon origin and rapid pan-Amazonian diversification of a lowland primate, Molecular Phlygenetics and Evolution, 82, 436-454.

Martins, F. M., A. R. Templeton, A. C. O. Pavan, B. C. Kohlbach, and J. S. Morgante (2009), Phylogeography of the common vampire bat (Desmodus rotundus): Marked population structure, Neotropical Pleistocene vicariance and incongruence between nuclear and mtDNA markers, BMC Evolutionary Biology, 9, e294.

Mercer, J. M., and V. L. Roth (2003), The effects of Cenozoic global change on squirrel phylogeny, Science, 299, 1568-1572.

Morales-Jiminez, A. L., T. Disotell, and A. Di Fiore (2015), Revisiting the phylogenetic relationships, biogeography, and taxonomy of spider monekys (genus Ateles) in light of new molecular data, Molecular Phylogenetics and Evolution, 82, 467-483.

Pava, A. C., F. E. Martins, and J. S. Morgante (2013), Evolutionary history of bulldog bats (genus Noctilo): recent diversification and the rold of the Caribbean in Neotropical biogeography, Biological Journal of the Linnean Society, 108, 210-224.

Perini, F. A., C. A. M. Russo, and C. G. Schrago (2009), The evolution of South American endemic canids: a history of rapid diversification and morphological parallelism, Journal of Evolutionary Biology, $23,311-322$.

Stadelmann, B., L.-K. Lin, T. H. Kunz, and M. Reudi (2007), Molecular phylogeny of New World Myotis (Chiroptera, Vespertilionidae) inferred from mitchondrial and nulcear DNA genes, Molecular Phylogenetics and Evolution, 43, 32-48. 